\title{
Infrared Spectroscopy of Arginine Cation Complexes: Direct Observation of Gas-phase Zwitterions
}

\author{
Matthew W. Forbes, ${ }^{a}$ Matthew F. Bush, ${ }^{b}$ Nick C. Polfer, ${ }^{c, e}$ Jos Oomens, ${ }^{c}$ Robert C. \\ Dunbar, ${ }^{d}$ Evan R. Williams ${ }^{b}$ and Rebecca A. Jockusch ${ }^{a^{*}}$ \\ ${ }^{a}$ Department of Chemistry, University of Toronto, Toronto, ON, Canada \\ ${ }^{\boldsymbol{b}}$ Department of Chemistry, University of California, Berkeley, California, USA \\ c FOM Institute for Plasma Physics "Rijnhuizen", Nieuwegein, The Netherlands \\ ${ }^{d}$ Chemistry Department, Case Western Reserve University, Cleveland, Ohio, USA \\ $e^{e}$ present address: Department of Chemistry, University of Florida, Gainesville, Florida, USA
}

\section{Supporting Information}

*Corresponding Author: e-mail: rebecca.jockusch@utoronto.ca 


\section{Table of Contents}

Table 1. Summary of structure names

Pages

S3 $[\mathrm{ArgOMe}+\mathrm{H}]^{+}$

Table 2. MP2//B3LYP energies

S4

Scheme 1. B3LYP optimized geometries

S5

Figure 1. B3LYP vibrational spectra

S6-S8

$[\mathrm{Arg}+\mathrm{H}]^{+}$

Table 3. MP2//B3LYP energies

S9

Scheme 2. B3LYP optimized geometries

S10-S11

Figure 2. B3LYP vibrational spectra

S12-S16 $[\mathrm{Arg}+\mathrm{Li}]^{+}$

Table 4. MP2//B3LYP energies

S17

Scheme 3. B3LYP optimized geometries

S18-S19

Figure 3. B3LYP vibrational spectra

S20-S25 $[\mathrm{Arg}+\mathrm{Na}]^{+}$

Table 5a. MP2//MP2 energies

$S 26$

Table 5b. MP2//B3LYP energies

S27-S28

Scheme 4. B3LYP optimized geometries

S29-S31

Figure 4. B3LYP vibrational spectra

S32-S42 $[\mathrm{Arg}+\mathrm{K}]^{+}$

Table 6. MP2//B3LYP energies

$S 43$

Scheme 5. B3LYP optimized geometries

S44-S45

Figure 5. B3LYP vibrational spectra $[\mathrm{Arg}+\mathrm{Rb}]^{+}$

Table 7. MP2//B3LYP energies

S46-S50

Scheme 6. B3LYP optimized geometries

Figure 6. B3LYP vibrational spectra

S51

S52-S53

S54-S58 $[\mathrm{Arg}+\mathrm{Cs}]^{+}$

Table 8. MP2//B3LYP energies

S59

Scheme 7. B3LYP optimized geometries

S60-S61

Figure 7. B3LYP vibrational spectra $[\mathrm{Arg}+\mathrm{Ag}]^{+}$

S62-S66

Table 9a. MP2//B3LYP energies $\quad S 67$

Table 9b. MP2//MPW1PW91 energies $\quad S 67$

Scheme 8. MPW1PW91 optimized geometries $\quad S 68$

Figure 8a. B3LYP vibrational spectra $\quad S 69$

Figure 8b. MPW1PW91 vibrational spectra $\quad S 70$ 
Table 1. A summary of the conformer names used by Bush et al. ${ }^{a}$ and corresponding names used in the present work. Conformers are referred to by their letter names in the text but are organized by their numbered codes in the tables of supporting information. In a few cases (based on MP2 single-point energies), a similar conformer from a particular family was found to be lower in energy than that reported by Bush et al. and have been denoted as, for example, SB_F'.

${ }^{a}$ J Am Chem Soc (2007) 129, 1612

\begin{tabular}{|c|c|c|c|}
\hline Bush et al. $^{a}$ & Name & $\begin{array}{l}\text { Structures } \\
\text { Calculated }\end{array}$ & $\begin{array}{c}\text { Ion } \\
\text { Complex(es) }\end{array}$ \\
\hline A & CS_A & CS_C039 & {$[\mathrm{Arg}+\mathrm{M}]^{+} \mathrm{M}=\mathrm{Li}^{+}, \mathrm{Na}^{+}, \mathrm{K}^{+}, \mathrm{Rb}^{+}, \mathrm{Cs}^{+}, \mathrm{Ag}^{+}$} \\
\hline $\mathrm{B}$ & CS_B & CS_C012 & {$[\mathrm{Arg}+\mathrm{M}]^{+} \mathrm{M}=\mathrm{Li}^{+}, \mathrm{Na}^{+}, \mathrm{K}^{+}, \mathrm{Rb}^{+}, \mathrm{Cs}^{+}, \mathrm{Ag}^{+}$} \\
\hline C & CS_C & CS_00C & {$[\mathrm{Arg}+\mathrm{Ag}]^{+}$} \\
\hline $\mathrm{D}$ & $\begin{array}{l}\text { CS_D } \\
\text { CS_D } \\
\text { SB D }\end{array}$ & $\begin{array}{l}\text { CS_003 } \\
\text { CS_001 } \\
\text { SB 003 }\end{array}$ & $\begin{array}{l}{[\mathrm{ArgOMe}+\mathrm{H}]^{+}} \\
{[\mathrm{Arg}+\mathrm{H}]^{+}} \\
{[\mathrm{Arg}+\mathrm{M}]^{+} \mathrm{M}=\mathrm{Li}^{+}, \mathrm{Na}^{+}, \mathrm{K}^{+}, \mathrm{Rb}^{+}, \mathrm{Cs}^{+}, \mathrm{Ag}^{+}}\end{array}$ \\
\hline$E$ & $\mathrm{SB} E$ & SB_009 & {$[\mathrm{Arg}+\mathrm{M}]^{+} \mathrm{M}=\mathrm{Li}^{+}, \mathrm{Na}^{+}, \mathrm{K}^{+}, \mathrm{Rb}^{+}, \mathrm{Cs}^{+}$} \\
\hline $\mathrm{F}$ & $\begin{array}{l}\text { SB_F } \\
\text { SB_F }\end{array}$ & $\begin{array}{l}\text { SB_032 } \\
\text { SB_026 }\end{array}$ & {$[\mathrm{Arg}+\mathrm{M}]^{+} \mathrm{M}=\mathrm{Li}^{+}, \mathrm{Na}^{+}, \mathrm{K}^{+}, \mathrm{Rb}^{+}, \mathrm{Cs}^{+}$} \\
\hline G & $\begin{array}{l}\text { SB_G } \\
\text { SB_G' }\end{array}$ & $\begin{array}{l}\text { SB_001 } \\
\text { SB_002 }\end{array}$ & {$[\mathrm{Arg}+\mathrm{M}]^{+} \mathrm{M}=\mathrm{Li}^{+}, \mathrm{Na}^{+}, \mathrm{K}^{+}, \mathrm{Rb}^{+}, \mathrm{Cs}^{+}$} \\
\hline $\mathrm{H}$ & $-\overline{S B} \mathrm{H}^{\prime}$ & SB_011 & {$[\mathrm{Arg}+\mathrm{M}]^{+} \mathrm{M}=\mathrm{Li}^{+}, \mathrm{Na}^{+}, \mathrm{K}^{+}, \mathrm{Rb}^{+}, \mathrm{Cs}^{+}$} \\
\hline $\mathrm{I}$ & $\begin{array}{l}\text { SB_I } \\
\text { SB_I' }\end{array}$ & $\begin{array}{l}\text { SB_031 } \\
\text { SB_033 }\end{array}$ & {$[\mathrm{Arg}+\mathrm{M}]^{+} \mathrm{M}=\mathrm{Li}^{+}, \mathrm{Na}^{+}, \mathrm{K}^{+}, \mathrm{Rb}^{+}, \mathrm{Cs}^{+}$} \\
\hline $\mathrm{J}$ & SB_J & SB_006 & {$[\mathrm{Arg}+\mathrm{M}]^{+} \mathrm{M}=\mathrm{Li}^{+}, \mathrm{Na}^{+}, \mathrm{K}^{+}, \mathrm{Rb}^{+}, \mathrm{Cs}^{+}$} \\
\hline- & $\mathrm{SB} \_\mathrm{K}$ & SB_013 & {$[\mathrm{Arg}+\mathrm{H}]^{+}$} \\
\hline- & CS_L & CS_C009 & {$[\mathrm{Arg}+\mathrm{M}]^{+} \mathrm{M}=\mathrm{Li}^{+}, \mathrm{Na}^{+}, \mathrm{K}^{+}, \mathrm{Rb}^{+}, \mathrm{Cs}^{+}$} \\
\hline - & CS_M & CS_00A & {$[\mathrm{Arg}+\mathrm{Ag}]^{+}$} \\
\hline
\end{tabular}


Table 2. Summary of electronic and thermal energies calculated for [ArgOMe+H] $]^{+}$.

\begin{tabular}{|c|c|c|c|c|c|c|c|c|}
\hline & & & B3LYP & & MP2 & & & \\
\hline ArgOMe & Name & Converge & $\begin{array}{c}\Delta E_{\mathrm{o}} / \\
\mathrm{kJ} \text { mol-1 }\end{array}$ & $\begin{array}{c}\Delta E_{\mathrm{o}} / \\
\mathrm{kJ} \text { mol-1 }\end{array}$ & $\begin{array}{c}\Delta \mathrm{H}(298) / \\
\mathrm{kJ} \text { mol-1 }\end{array}$ & $\begin{array}{c}\Delta G(298) / \\
\text { kJ mol-1 }\end{array}$ & SCF E / h & MP2 E / h \\
\hline CS_003 & CS_D & Min & 0.0 & 0.0 & 0.0 & 0.0 & -646.3219 & -644.8198 \\
\hline CS_001 & & Min & 0.2 & 0.2 & 0.0 & 0.6 & -646.3219 & -644.8198 \\
\hline CS_002 & & Min & 10.4 & 9.2 & 9.0 & 9.6 & -646.3180 & -644.8164 \\
\hline CS_062 & & Min & 12.9 & 15.3 & 17.5 & 10.8 & -646.3154 & -644.8124 \\
\hline CS_008 & & Min & 18.7 & 16.0 & 17.1 & 12.8 & -646.3143 & -644.8132 \\
\hline CS_014 & & Min & 14.7 & 17.0 & 18.3 & 12.3 & -646.3154 & -644.8124 \\
\hline CS_004 & CS_E & Min & 11.0 & 19.1 & 21.0 & 13.1 & -646.3166 & -644.8114 \\
\hline CS_027 & & Min & 22.2 & 19.6 & 20.8 & 17.0 & -646.3128 & -644.8117 \\
\hline CS_011 & & Min & 18.4 & 20.1 & 21.9 & 14.5 & -646.3138 & -644.8111 \\
\hline CS_010 & & Min & 19.0 & 22.8 & 24.8 & 18.6 & -646.3134 & -644.8099 \\
\hline CS_013 & & Min & 20.3 & 23.4 & 24.3 & 19.5 & -646.3139 & -644.8106 \\
\hline CS_036 & & Min & 26.7 & 25.1 & 26.9 & 20.2 & -646.3107 & -644.8092 \\
\hline CS_006 & & Min & 21.7 & 26.1 & 26.4 & 23.2 & -646.3134 & -644.8096 \\
\hline CS_039 & & Min & 24.0 & 27.2 & 27.7 & 21.8 & -646.3125 & -644.8092 \\
\hline CS_037 & & Min & 27.2 & 27.6 & 29.8 & 21.9 & -646.3105 & -644.8083 \\
\hline CS_033 & & Min & 22.9 & 27.8 & 28.7 & 25.9 & -646.3126 & -644.8086 \\
\hline CS_035 & & Min & 24.6 & 28.6 & 29.9 & 25.8 & -646.3117 & -644.8081 \\
\hline CS_005 & & Min & 29.4 & 29.4 & 29.7 & 26.6 & -646.3105 & -644.8084 \\
\hline CS_012 & & Min & 29.2 & 30.5 & 31.5 & 28.4 & -646.3104 & -644.8078 \\
\hline CS_029 & & Min & 29.8 & 31.2 & 31.7 & 28.7 & -646.3103 & -644.8077 \\
\hline CS_067 & & Min & 26.8 & 31.7 & 33.8 & 26.6 & -646.3108 & -644.8069 \\
\hline CS_043 & & Min & 29.0 & 33.6 & 34.2 & 30.9 & -646.3106 & -644.8068 \\
\hline CS_047 & & Min & 27.3 & 35.2 & 36.5 & 30.7 & -646.3110 & -644.8060 \\
\hline CS_061 & & Min & 33.5 & 36.8 & 37.4 & 33.9 & -646.3088 & -644.8055 \\
\hline CS_058 & & Min & 32.9 & 37.5 & 37.7 & 34.5 & -646.3091 & -644.8053 \\
\hline CS_063 & & Min & 30.5 & 39.2 & 41.8 & 30.5 & -646.3089 & -644.8034 \\
\hline
\end{tabular}

Geometry Optimization

Thermal Corrections

MP2 Single-point
B3LYP/6-31+g(d,p)

B3LYP/6-31+g(d,p)

MP2/6-311++g(2d,2p)//B3LYP/6-31+g(d,p) 
Scheme 1. Geometry optimized conformers of ArgOMe (B3LYP/6-31+g(d,p)).

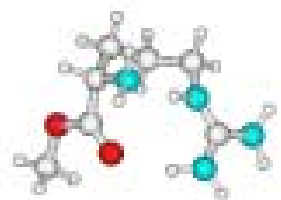

CS_001

\&. a

CS_010

$8^{2} 0^{2}=0$

CS_033

2.8.

CS_058

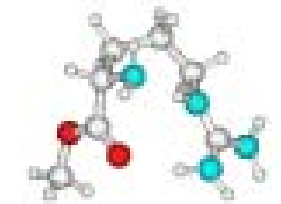

CS_002

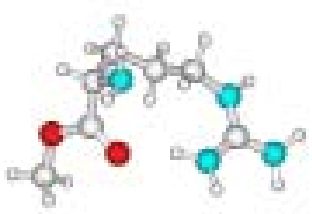

CS_011

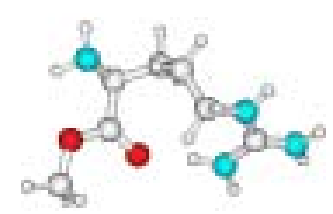

CS_035

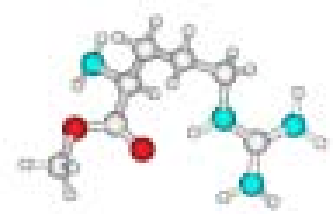

CS_061

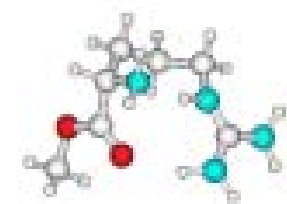

CS_003

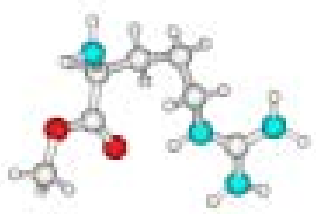

$$
\text { CS_012 }
$$

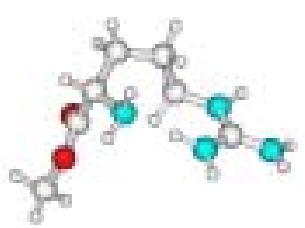

CS_036

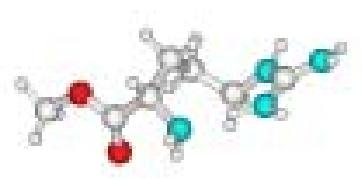

CS_062
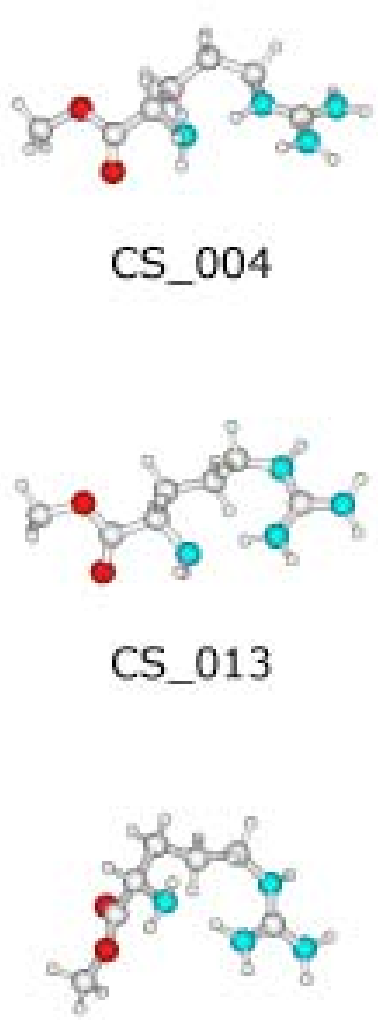

CS_004

$$
\text { CS_013 }
$$

$$
\text { CS_037 }
$$

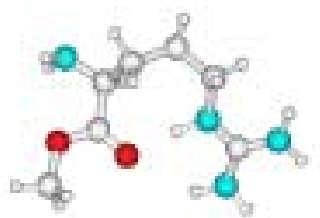

CS_063

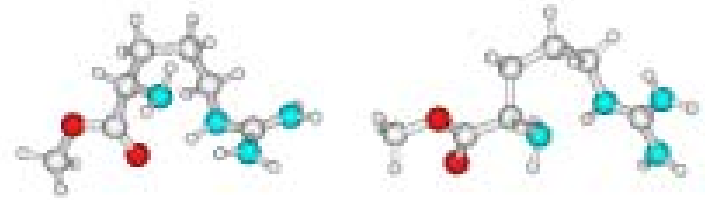

$$
\text { CS_005 }
$$

CS_006

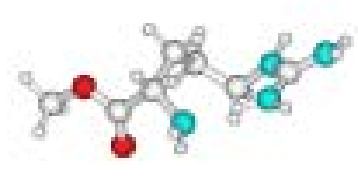

CS_014

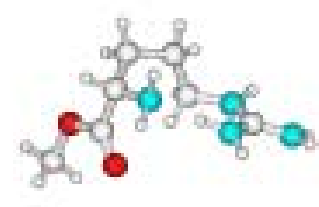

$$
\text { CS_027 }
$$
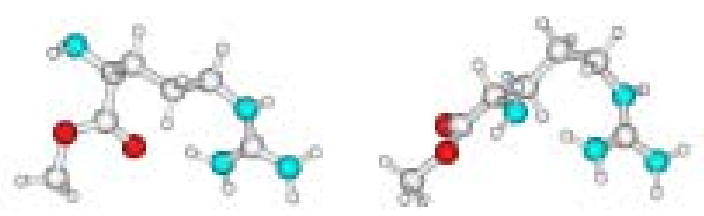

CS_039

CS_043

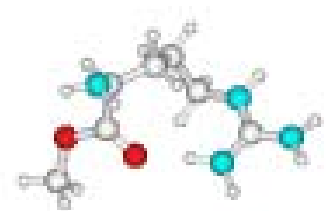

CS_067

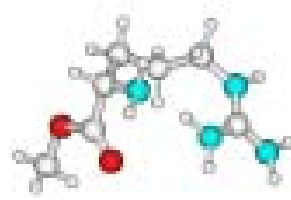

CS_008

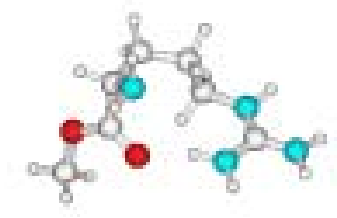

CS_029

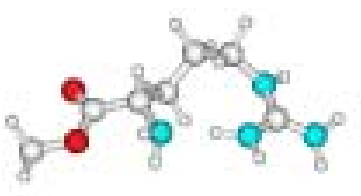

CS_047 
Figure 1. Vibrational spectra for $[\mathrm{ArgOMe}+\mathrm{H}]^{+}$conformers from B3LYP/6-31+g(d,p) calculations. Spectra were constructed using a Gaussian convolution (FWHM=20 $\mathrm{cm}-1)$ and a scaling factor of 0.98 .
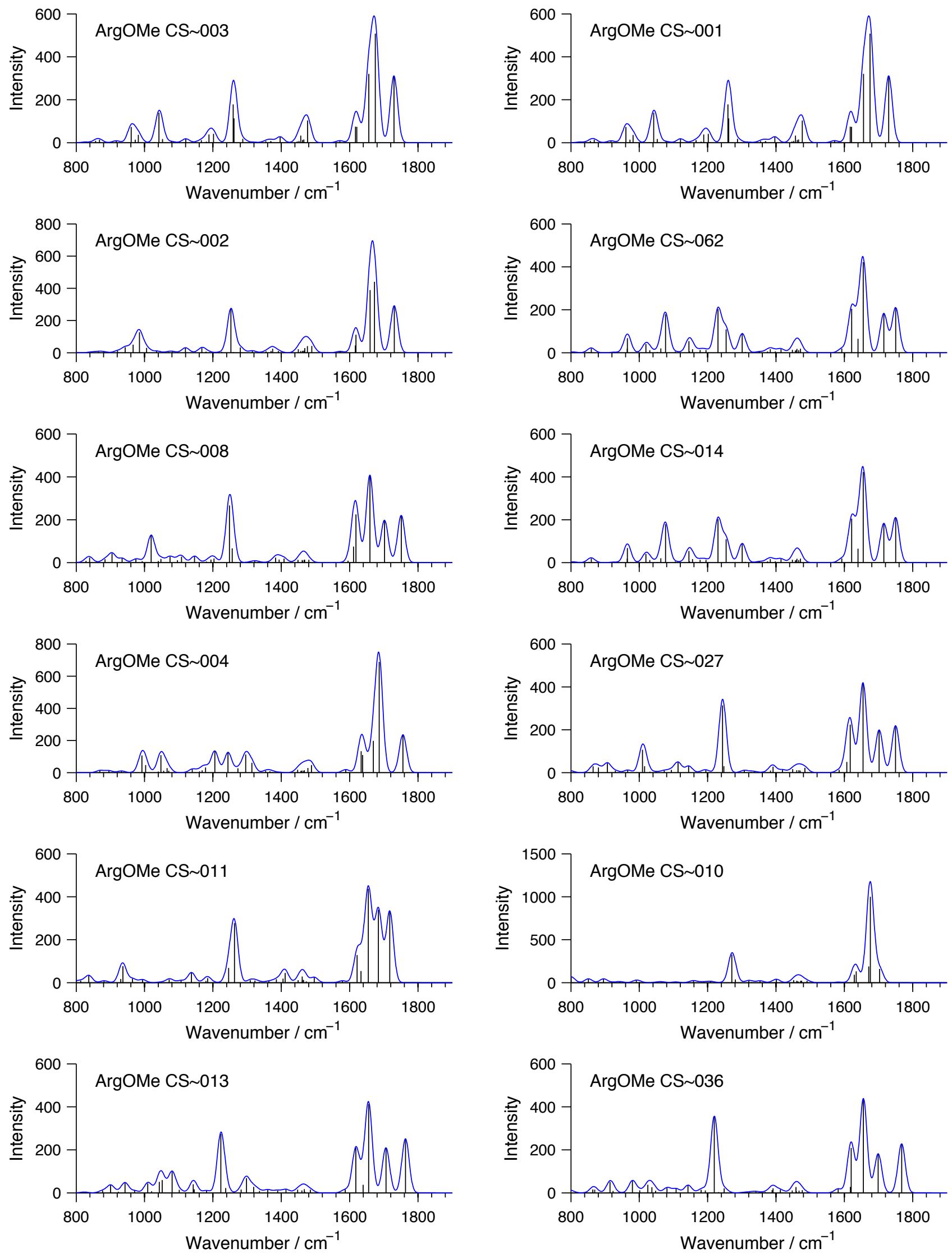


\section{Figure 1continued.}
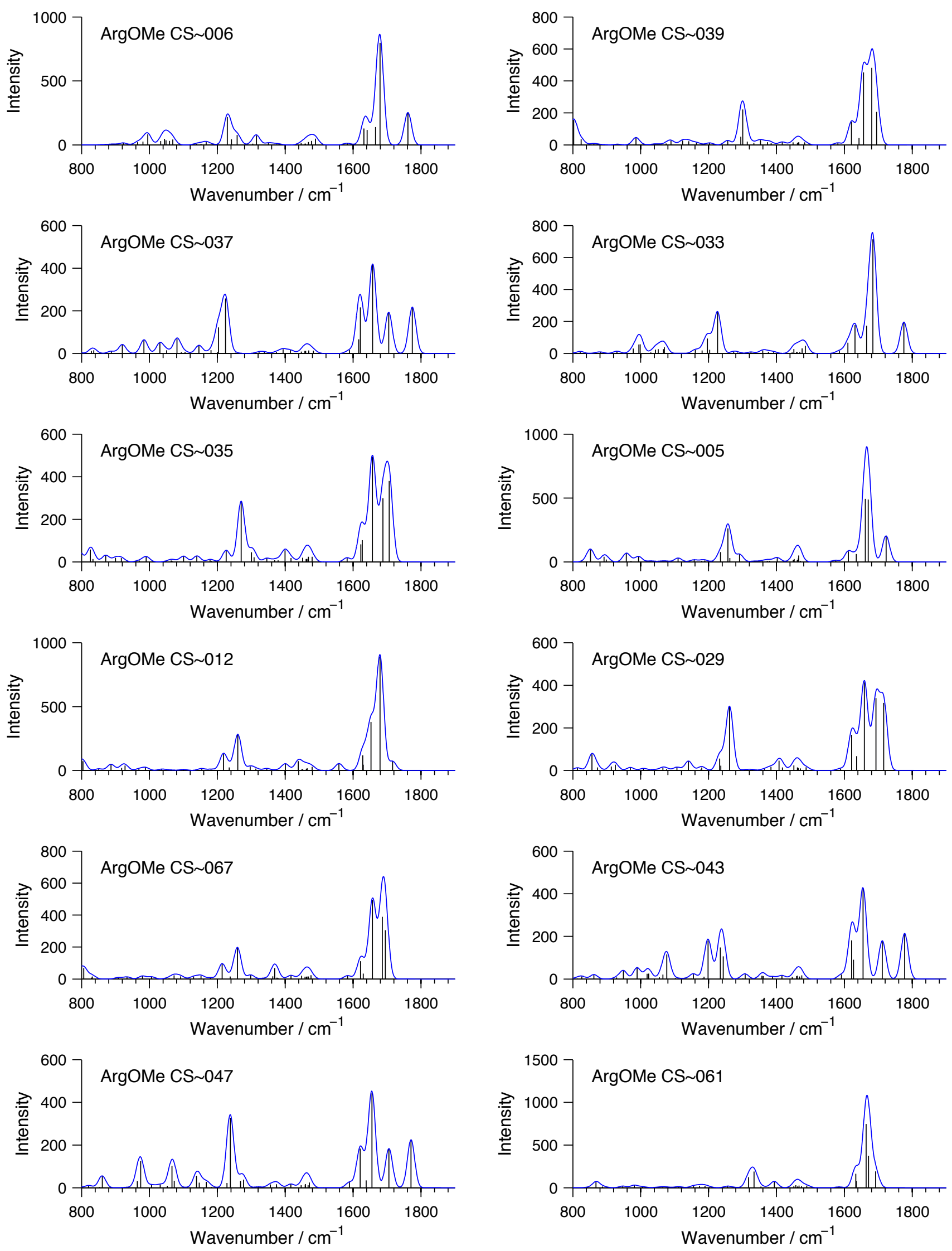
Figure 1continued.
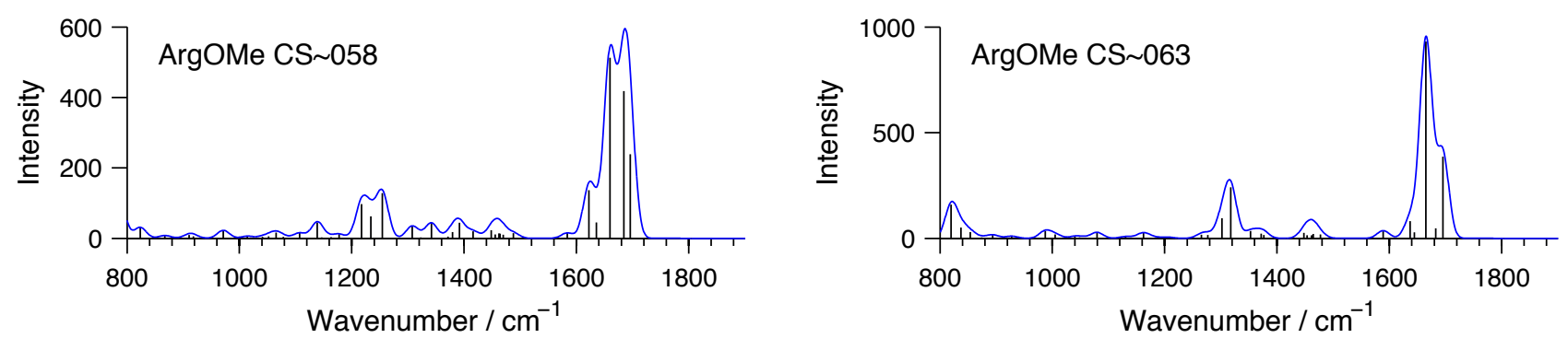
Table 3. Summary of electronic and thermal energies calculated for $[\mathrm{Arg}+\mathrm{H}]^{+}$.

\begin{tabular}{|c|c|c|c|c|c|c|c|c|}
\hline & & & B3LYP & & MP2 & & & \\
\hline ArgH & Name & Converge & $\begin{array}{c}\Delta E_{o} / \\
k J \text { mol-1 }\end{array}$ & $\begin{array}{c}\Delta E_{o} / \\
k J \text { mol-1 }\end{array}$ & $\begin{array}{c}\Delta H(298) / \\
\text { kJ mol-1 }\end{array}$ & $\begin{array}{c}\Delta G(298) / \\
\text { kJ mol-1 }\end{array}$ & SCF E / h & MP2 E / h \\
\hline CS_004 & CS_D & Min & 0.0 & 0.0 & 0.0 & 0.0 & -607.0149 & -605.6285 \\
\hline CS_001 & CS_D & Min & 0.0 & 0.0 & 0.0 & 0.0 & -607.0149 & -605.6285 \\
\hline CS_002 & & Min & 9.4 & 7.9 & 8.1 & 7.4 & -607.0112 & -605.6255 \\
\hline CS_012 & & Min & 17.5 & 14.7 & 15.8 & 11.5 & -607.0078 & -605.6226 \\
\hline SB_010 (CS) & & Min & 11.4 & 15.5 & 16.2 & 13.8 & -607.0105 & -605.6226 \\
\hline CS_003 & & Min & 10.5 & 18.0 & 20.0 & 12.0 & -607.0098 & -605.6206 \\
\hline CS_018 & & Min & 16.3 & 18.3 & 18.6 & 16.3 & -607.0086 & -605.6215 \\
\hline CS_008 & & Min & 22.6 & 20.0 & 21.5 & 16.1 & -607.0055 & -605.6202 \\
\hline SB_002 (CS) & & Min & 10.2 & 20.4 & 22.4 & 14.6 & -607.0100 & -605.6197 \\
\hline SB_001 (CS) & & Min & 10.2 & 20.4 & 22.4 & 14.6 & -607.0100 & -605.6197 \\
\hline CS_023 & & Min & 20.2 & 21.5 & 22.6 & 19.2 & -607.0068 & -605.6200 \\
\hline CS_080 & & Min & 20.2 & 21.5 & 22.6 & 19.2 & -607.0068 & -605.6200 \\
\hline SB_008 (CS) & & Min & 14.0 & 21.5 & 22.7 & 17.7 & -607.0092 & -605.6200 \\
\hline CS_016 & & Min & 19.3 & 21.8 & 22.6 & 18.6 & -607.0074 & -605.6201 \\
\hline CS_058 & & Min & 18.5 & 22.4 & 24.3 & 17.0 & -607.0069 & -605.6190 \\
\hline CS_014 & & Min & 19.4 & 23.0 & 25.3 & 18.4 & -607.0062 & -605.6185 \\
\hline CS_051 & & Min & 19.4 & 23.0 & 25.3 & 18.4 & -607.0062 & -605.6185 \\
\hline CS_041 & & Min & 24.7 & 24.6 & 26.3 & 18.9 & -607.0045 & -605.6182 \\
\hline SB_018(CS) & & Min & 20.0 & 25.3 & 25.7 & 23.4 & -607.0070 & -605.6187 \\
\hline CS_060 & & Min & 21.6 & 25.7 & 28.3 & 20.4 & -607.0052 & -605.6172 \\
\hline CS_028 & & Min & 27.6 & 25.8 & 26.3 & 24.0 & -607.0043 & -605.6186 \\
\hline CS_081 & & Min & 22.2 & 26.2 & 28.6 & 21.3 & -607.0051 & -605.6172 \\
\hline CS_034 & & Min & 22.2 & 26.2 & 28.6 & 21.3 & -607.0051 & -605.6172 \\
\hline CS_036 & & Min & 22.8 & 26.3 & 27.7 & 23.4 & -607.0056 & -605.6179 \\
\hline CS_078 & & Min & 20.9 & 26.6 & 29.2 & 21.4 & -607.0055 & -605.6170 \\
\hline CS_005 & & Min & 19.6 & 28.6 & 30.6 & 20.1 & -607.0062 & -605.6165 \\
\hline CS_009 & & Min & 29.6 & 28.9 & 30.4 & 24.7 & -607.0032 & -605.6171 \\
\hline CS_015 & & Min & 27.6 & 28.9 & 30.8 & 24.1 & -607.0034 & -605.6165 \\
\hline CS_082 & & Min & 28.3 & 29.3 & 31.4 & 24.8 & -607.0033 & -605.6166 \\
\hline SB_017 (CS) & & Min & 24.1 & 29.8 & 30.4 & 27.6 & -607.0055 & -605.6170 \\
\hline CS_038 & & Min & 25.9 & 29.9 & 30.9 & 27.7 & -607.0044 & -605.6166 \\
\hline CS_017 & & Min & 27.2 & 30.1 & 31.0 & 25.8 & -607.0045 & -605.6170 \\
\hline CS_022 & & Min & 26.8 & 31.4 & 32.9 & 23.3 & -607.0041 & -605.6160 \\
\hline CS_037 & & Min & 29.6 & 31.9 & 33.8 & 27.5 & -607.0028 & -605.6156 \\
\hline CS_055 & & Min & 27.4 & 32.7 & 34.0 & 30.3 & -607.0038 & -605.6155 \\
\hline CS_054 & & Min & 27.8 & 33.8 & 36.9 & 27.8 & -607.0025 & -605.6139 \\
\hline SB_012 (CS) & & Min & 32.3 & 35.4 & 35.9 & 31.2 & -607.0027 & -605.6152 \\
\hline CS_040 & & Min & 35.1 & 35.8 & 37.4 & 30.7 & -607.0007 & -605.6140 \\
\hline CS_053 & & Min & 32.5 & 37.8 & 39.3 & 34.0 & -607.0019 & -605.6135 \\
\hline CS_059 & & Min & 32.6 & 38.2 & 41.1 & 31.4 & -607.0008 & -605.6123 \\
\hline CS_044 & & Min & 35.3 & 38.8 & 41.1 & 32.9 & -607.0003 & -605.6126 \\
\hline CS_084 & & Min & 36.4 & 41.6 & 43.7 & 37.4 & -607.0001 & -605.6118 \\
\hline CS_083 & & Min & 44.5 & 42.6 & 42.1 & 41.9 & -606.9984 & -605.6128 \\
\hline CS_052 & & Min & 33.8 & 42.6 & 44.7 & 36.1 & -607.0009 & -605.6112 \\
\hline CS_085 & & Min & 39.6 & 44.5 & 45.4 & 42.0 & -606.9995 & -605.6113 \\
\hline CS_062 & & Min & 47.0 & 50.2 & 53.6 & 41.3 & -606.9954 & -605.6078 \\
\hline SB_013 & SB_K & Min & 68.1 & 60.6 & 60.6 & 60.2 & -606.9894 & -605.6059 \\
\hline CS_057 & & Min & 62.9 & 63.1 & 66.5 & 52.6 & -606.9894 & -605.6030 \\
\hline SB_022 & & Min & 74.2 & 68.5 & 67.9 & 69.4 & -606.9860 & -605.6019 \\
\hline
\end{tabular}

Geometry Optimization

Thermal Corrections

MP2 Single-point
$B 3 L Y P / 6-31+g(d, p)$

B3LYP/6-31+g(d,p)

MP2/6-311++g(2d,2p)//B3LYP/6-31+g(d,p)
Salt Bridge 
Scheme 2a. Geometry optimized conformers of $[\mathrm{Arg}+\mathrm{H}]^{+}(\mathrm{B} 3 \mathrm{LYP} / 6-31+\mathrm{g}(\mathrm{d}, \mathrm{p}))$.
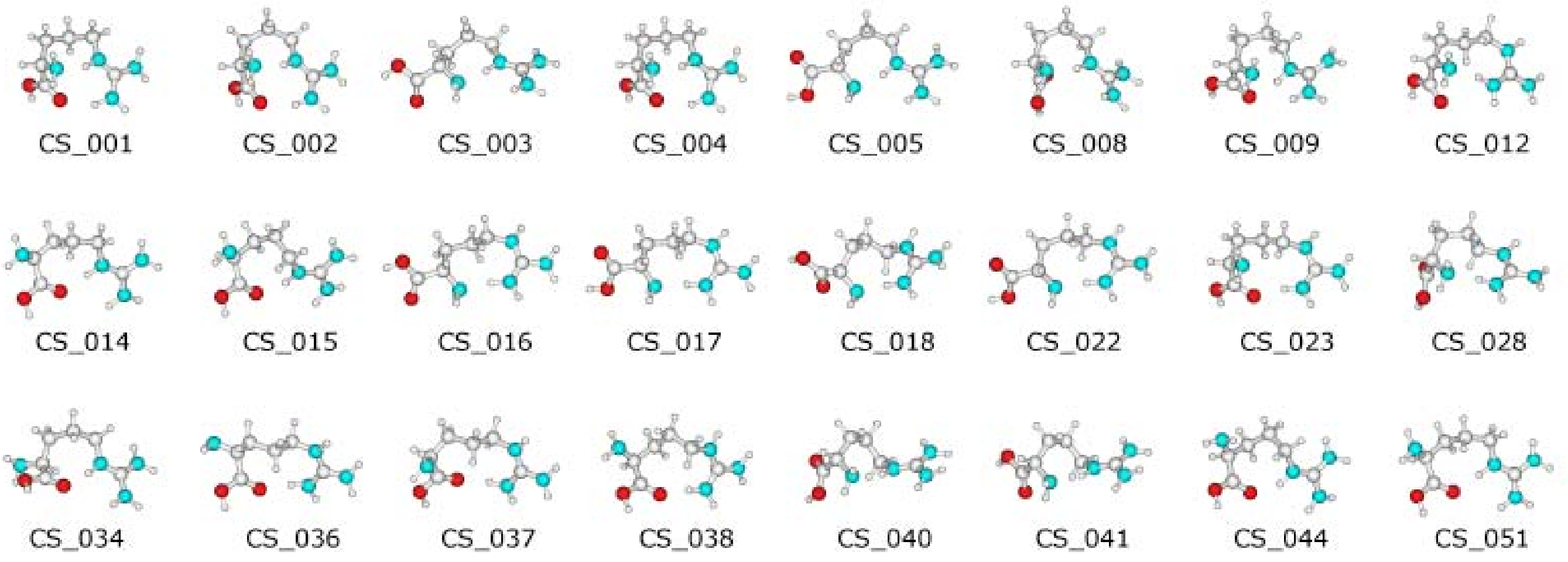

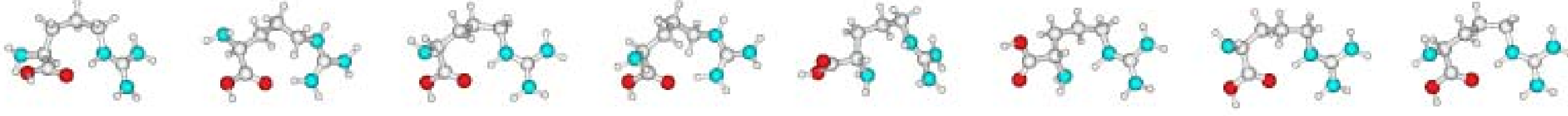

$$
\text { CS_052 CS_053 CS_054 CS_055 CS_057 CS_058 CS_059 }
$$

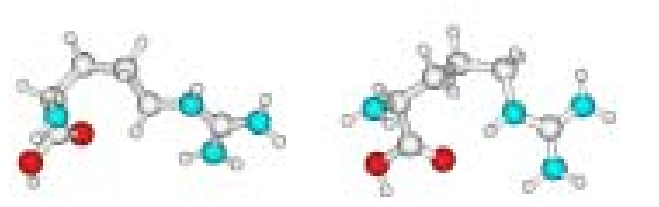
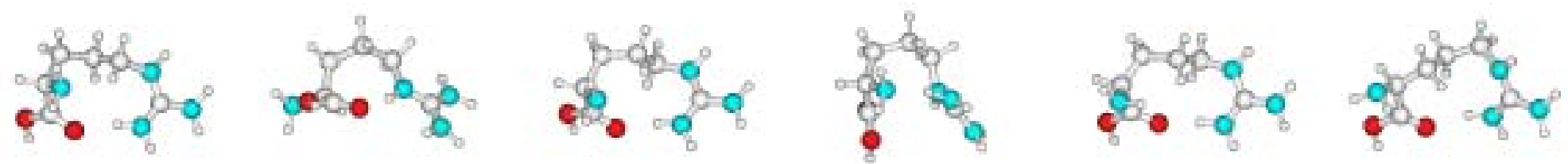

$$
\text { CS_062 }
$$

$$
\text { CS_078 }
$$

CS_080

CS_081

CS_082

CS_083

CS_084

CS_085 
Scheme $2 b$. Geometry optimized conformers of $[\mathrm{Arg}+\mathrm{H}]^{+}(\mathrm{B} 3 \mathrm{LYP} / 6-31+\mathrm{g}(\mathrm{d}, \mathrm{p}))$.

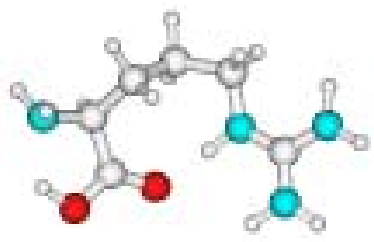

SB_001 (CS)

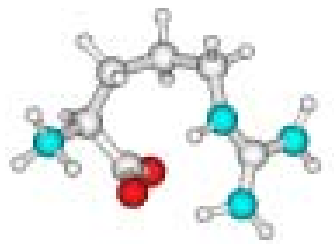

SB_013

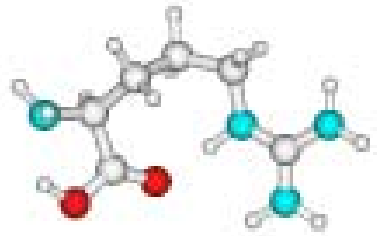

SB_002 (CS)

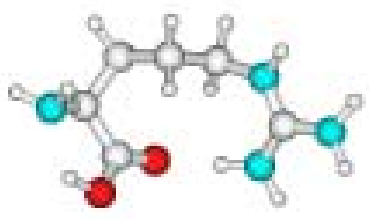

SB_017 (CS)

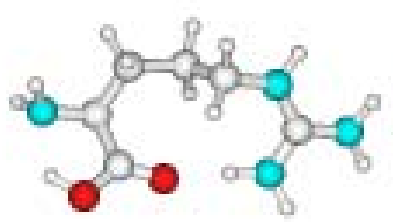

SB_008 (CS)

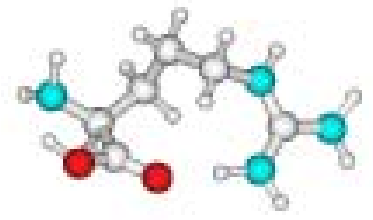

SB_018 (CS)

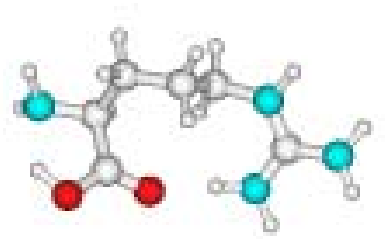

SB_010(CS)

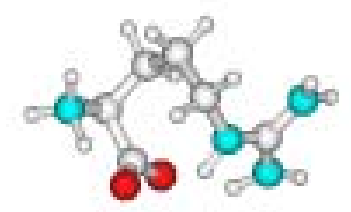

SB_022

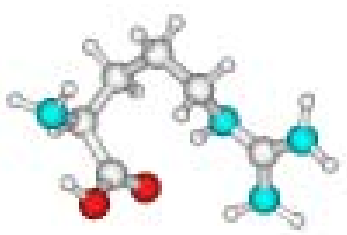

SB_012 (CS) 
Figure 2. Vibrational spectra for $[\mathrm{Arg}+\mathrm{H}]^{+}$conformers from $\mathrm{B} 3 \mathrm{LYP} / 6-31+\mathrm{g}(\mathrm{d}, \mathrm{p})$ calculations. Spectra were constructed using a Gaussian convolution (FWHM=20 $\mathrm{cm}-1$ ) and a scaling factor of 0.98 .
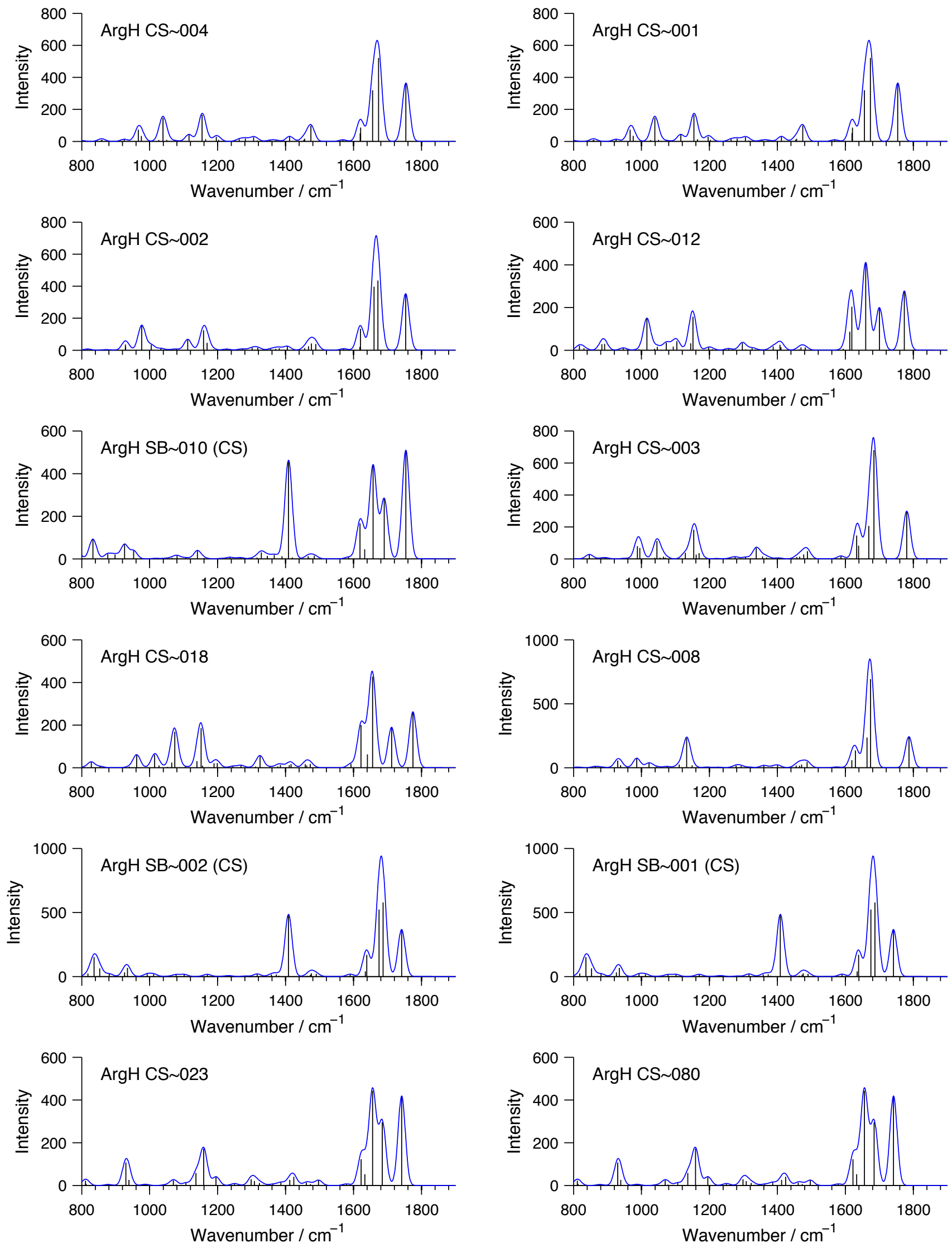
Figure 2 continued.
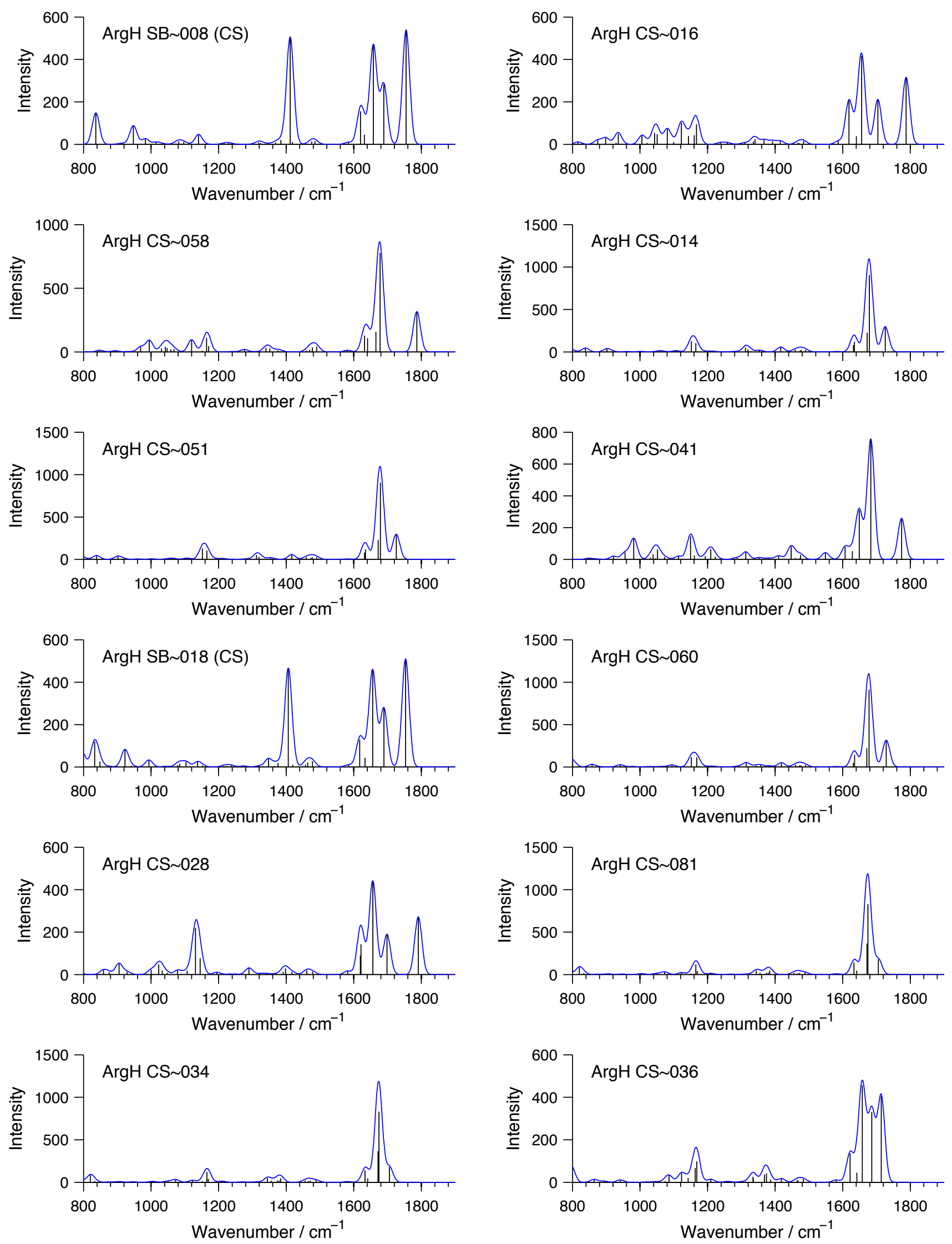
Figure 2 continued.
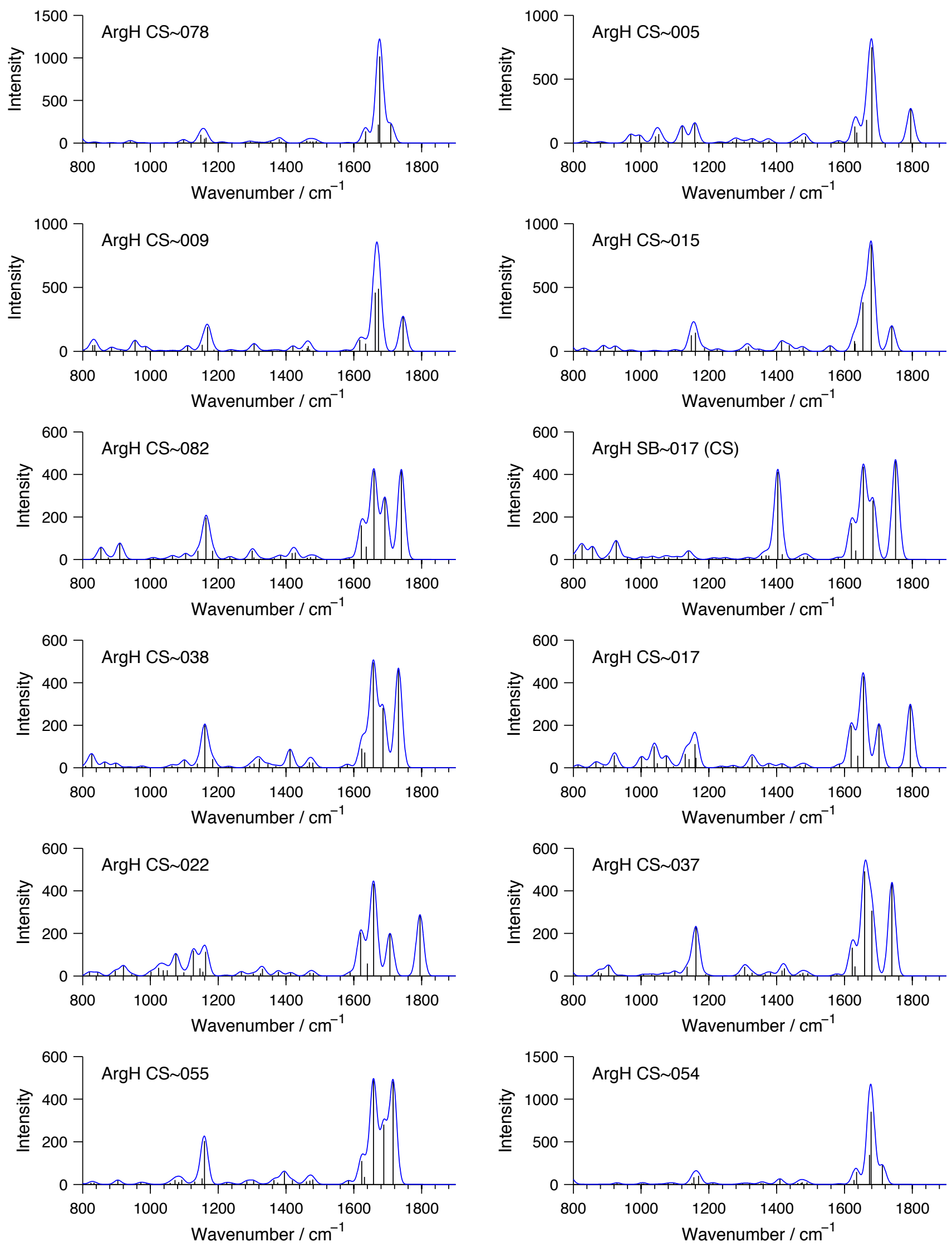
Figure 2 continued.
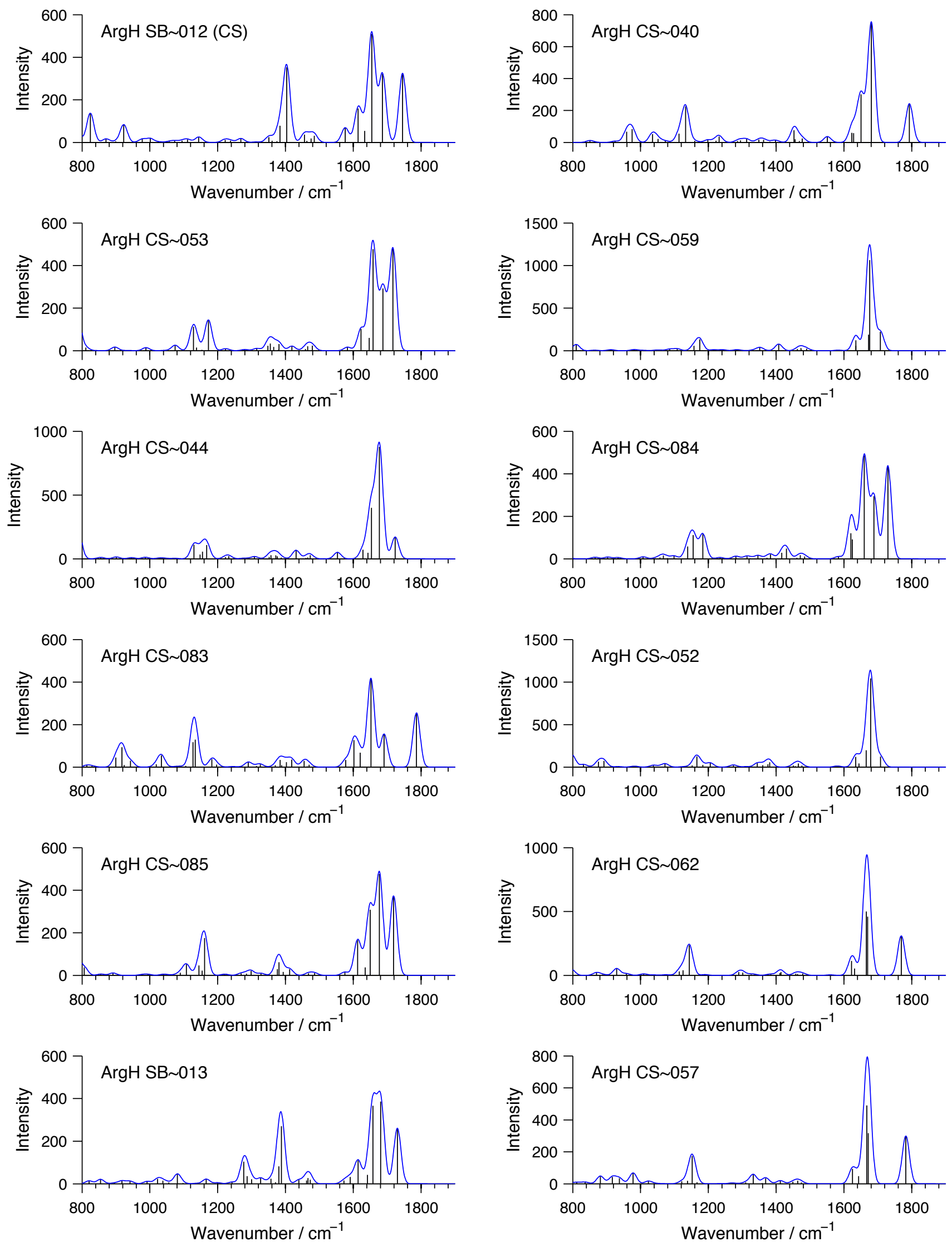
Figure 2 continued.

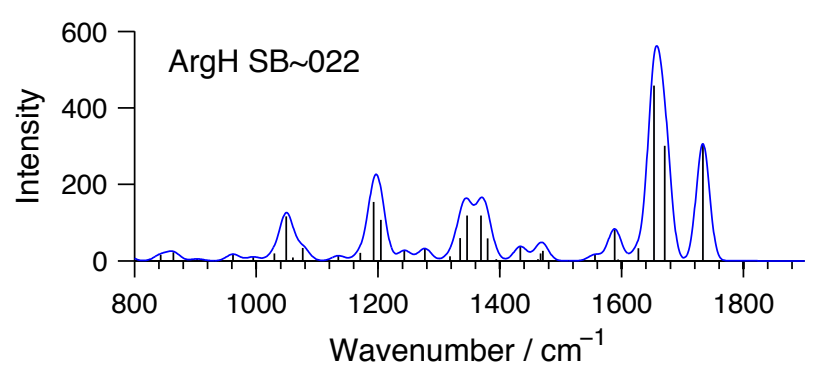


Table 4. Summary of electronic and thermal energies calculated for [Arg+Li] $]^{+}$.

\begin{tabular}{|c|c|c|c|c|c|c|c|c|}
\hline & & & B3LYP & & MP2 & & & \\
\hline ArgLi & Name & Converge & $\begin{array}{c}\Delta E_{\mathrm{o}} / \\
\text { kJ mol-1 }\end{array}$ & $\begin{array}{c}\Delta E_{o} / \\
k J \text { mol-1 }\end{array}$ & $\begin{array}{c}\Delta H(298) / \\
\text { kJ mol-1 }\end{array}$ & $\begin{array}{c}\Delta G(298) / \\
\text { kJ mol-1 }\end{array}$ & $\begin{array}{c}\text { B3LYP E } \\
/ \mathrm{h}\end{array}$ & $\begin{array}{c}\text { MP2 E } \\
/ \mathbf{h}\end{array}$ \\
\hline CS_C039 & CS_A & Min & 0.0 & 0.0 & 0.0 & 0.0 & -614.0286 & -612.5914 \\
\hline CS_Chs1 & & Min & 14.1 & 13.8 & 13.7 & 13.7 & -614.0233 & -612.5862 \\
\hline SB_003 & SB_D & Min & 15.9 & 15.0 & 15.4 & 14.0 & -614.0216 & -612.5847 \\
\hline SB_005 & & Min & 14.4 & 19.6 & 21.0 & 15.3 & -614.0215 & -612.5823 \\
\hline SB_001 & SB_G & Min & 11.0 & 21.3 & 22.9 & 16.0 & -614.0229 & -612.5818 \\
\hline SB_007 & & Min & 24.9 & 21.7 & 22.4 & 20.2 & -614.0180 & -612.5820 \\
\hline SB_002 & SB_G' & Min & 15.1 & 21.8 & 22.6 & 16.5 & -614.0221 & -612.5823 \\
\hline SB_033 & SB_I' & Min & 18.7 & 21.9 & 22.4 & 19.8 & -614.0208 & -612.5823 \\
\hline SB_023 & & Min & 19.2 & 23.2 & 24.3 & 19.4 & -614.0205 & -612.5817 \\
\hline CS_C012 & CS_B & Min & 14.1 & 24.8 & 25.9 & 21.6 & -614.0223 & -612.5810 \\
\hline SB_004 & & Min & 18.6 & 27.1 & 27.8 & 23.2 & -614.0209 & -612.5805 \\
\hline SB_009 & SB_E & Min & 22.6 & 29.7 & 32.2 & 22.8 & -614.0179 & -612.5780 \\
\hline SB_026 & SB_F' & Min & 28.1 & 30.1 & 31.0 & 26.6 & -614.0168 & -612.5788 \\
\hline SB_006 & SB_J & Min & 35.0 & 30.6 & 33.4 & 24.8 & -614.0132 & -612.5777 \\
\hline SB_032 & SB_F & Min & 25.6 & 30.9 & 31.8 & 26.8 & -614.0174 & -612.5781 \\
\hline SB_027 & & Min & 22.5 & 31.3 & 32.4 & 27.3 & -614.0193 & -612.5787 \\
\hline CS_C018 & & Min & 18.2 & 32.3 & 34.1 & 25.8 & -614.0204 & -612.5778 \\
\hline SB_044 & & Min & 32.6 & 32.4 & 33.7 & 28.1 & -614.0149 & -612.5778 \\
\hline CS_C025 & & Min & 18.3 & 32.7 & 34.3 & 26.9 & -614.0205 & -612.5777 \\
\hline SB_008 & & Min & 30.0 & 33.7 & 35.9 & 27.5 & -614.0153 & -612.5767 \\
\hline SB_013 & SB_K & Min & 34.0 & 33.9 & 36.8 & 28.4 & -614.0134 & -612.5762 \\
\hline CS_C006 & & Min & 28.8 & 34.9 & 35.4 & 31.6 & -614.0170 & -612.5774 \\
\hline SB_025 & & Min & 31.3 & 35.0 & 36.4 & 30.6 & -614.0154 & -612.5768 \\
\hline CS_C014 & & Min & 29.3 & 35.3 & 35.7 & 30.8 & -614.0168 & -612.5773 \\
\hline CS_C017 & & Min & 25.6 & 35.5 & 36.7 & 31.3 & -614.0177 & -612.5768 \\
\hline CS_003 & & Min & 43.7 & 35.7 & 35.0 & 36.7 & -614.0123 & -612.5781 \\
\hline CS_C003 & & Min & 25.7 & 36.3 & 37.8 & 30.8 & -614.0175 & -612.5762 \\
\hline SB_021 & & Min & 25.8 & 36.6 & 38.2 & 29.4 & -614.0174 & -612.5761 \\
\hline SB_018 & & Min & 25.8 & 36.6 & 38.3 & 29.5 & -614.0174 & -612.5760 \\
\hline SB_028 & & Min & 31.5 & 36.9 & 38.9 & 29.9 & -614.0150 & -612.5757 \\
\hline CS_Chs2 & & Min & 37.5 & 39.5 & 39.3 & 38.0 & -614.0141 & -612.5761 \\
\hline SB_014 & & Min & 42.5 & 39.7 & 40.9 & 36.2 & -614.0114 & -612.5752 \\
\hline SB_011 & SB_H' & Min & 39.6 & 40.4 & 43.2 & 34.8 & -614.0112 & -612.5736 \\
\hline SB_042 & & Min & 39.6 & 40.5 & 42.2 & 37.3 & -614.0116 & -612.5741 \\
\hline SB_050 & & Min & 41.0 & 41.0 & 42.5 & 37.7 & -614.0116 & -612.5744 \\
\hline CS_004 & & Min & 46.8 & 41.1 & 40.7 & 41.7 & -614.0106 & -612.5756 \\
\hline CS_001 & & Min & 44.2 & 43.2 & 43.6 & 42.2 & -614.0113 & -612.5745 \\
\hline CS_C010 & & Min & 45.3 & 43.3 & 43.5 & 40.5 & -614.0109 & -612.5744 \\
\hline CS_C043 & & Min & 30.8 & 43.4 & 44.3 & 40.0 & -614.0159 & -612.5739 \\
\hline CS_C026 & & Min & 40.0 & 43.4 & 44.2 & 40.9 & -614.0124 & -612.5739 \\
\hline SB_051 & & Min & 41.6 & 43.6 & 45.4 & 40.0 & -614.0112 & -612.5732 \\
\hline CS_C016 & & Min & 28.7 & 43.7 & 45.1 & 38.2 & -614.0164 & -612.5735 \\
\hline CS_C027 & & Min & 28.6 & 43.7 & 45.1 & 38.1 & -614.0164 & -612.5735 \\
\hline SB_062 & & Min & 46.4 & 43.9 & 46.3 & 39.5 & -614.0092 & -612.5729 \\
\hline CS_C040 & & Min & 40.0 & 43.9 & 44.4 & 41.1 & -614.0127 & -612.5739 \\
\hline CS_C021 & & Min & 37.3 & 44.3 & 44.6 & 41.3 & -614.0140 & -612.5741 \\
\hline CS_C044 & & Min & 33.7 & 44.6 & 45.2 & 40.5 & -614.0151 & -612.5737 \\
\hline CS_C001 & & Min & 36.4 & 45.0 & 45.8 & 40.2 & -614.0138 & -612.5733 \\
\hline CS_C009 & CS_L & Min & 44.5 & 45.2 & 45.9 & 42.4 & -614.0107 & -612.5732 \\
\hline SB_035 & & Min & 44.8 & 46.2 & 48.9 & 38.1 & -614.0098 & -612.5720 \\
\hline SB_015 & & Min & 43.8 & 46.3 & 49.4 & 39.6 & -614.0095 & -612.5713 \\
\hline CS_C004 & & Min & 36.5 & 46.9 & 47.9 & 39.8 & -614.0137 & -612.5725 \\
\hline SB 019 & & Min & 47.2 & 49.2 & 52.4 & 42.4 & -614.0081 & -612.5701 \\
\hline SB_016 & & Min & 46.2 & 49.5 & 52.7 & 42.3 & -614.0085 & -612.5701 \\
\hline CS_C013 & & Min & 45.0 & 50.3 & 51.1 & 46.7 & -614.0105 & -612.5712 \\
\hline CS_C008 & & Min & 45.0 & 50.4 & 51.1 & 46.8 & -614.0105 & -612.5712 \\
\hline CS_C007 & & Min & 49.6 & 50.5 & 51.2 & 47.1 & -614.0086 & -612.5710 \\
\hline CS_043 & & Min & 61.2 & 50.6 & 49.0 & 53.4 & -614.0063 & -612.5731 \\
\hline CS_016 & & Min & 66.1 & 53.3 & 52.0 & 56.2 & -614.0039 & -612.5715 \\
\hline CS_C023 & & Min & 54.8 & 53.3 & 53.4 & 51.0 & -614.0072 & -612.5705 \\
\hline CS_CS1 & & Min & 54.8 & 53.4 & 53.4 & 51.0 & -614.0072 & -612.5705 \\
\hline CS_C033 & & Min & 60.6 & 53.8 & 53.8 & 52.1 & -614.0048 & -612.5702 \\
\hline CS_C031 & & Min & 54.5 & 54.6 & 55.0 & 52.4 & -614.0071 & -612.5698 \\
\hline CS_022 & & Min & 72.4 & 61.7 & 61.8 & 61.3 & -614.0007 & -612.5676 \\
\hline CS_C035 & & Min & 60.3 & 62.4 & 62.7 & 59.6 & -614.0049 & -612.5669 \\
\hline
\end{tabular}

Geometry Optimization

Thermal Corrections

MP2 Single-point
B3LYP/6-31+g(d,p)

B3LYP $/ 6-31+g(d, p)$

MP2/6-311++g(2d,2p)//B3LYP/6-31+g(d,p) 
Scheme 3a. Geometry optimized conformers of $[\mathrm{Arg}+\mathrm{Li}]^{+}(\mathrm{B} 3 \mathrm{LYP} / 6-31+\mathrm{g}(\mathrm{d}, \mathrm{p}))$.

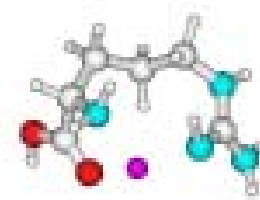

CS_001

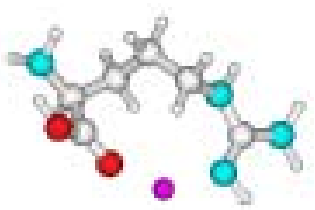

CS_C003

a.dog

CS_C012

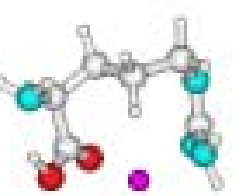

CS_C023

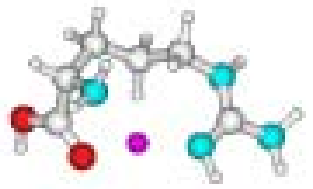

CS_C039

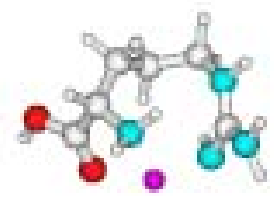

CS_003

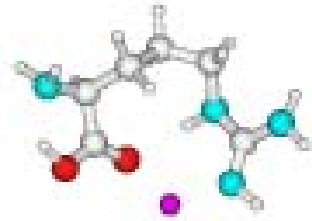

CS_C004

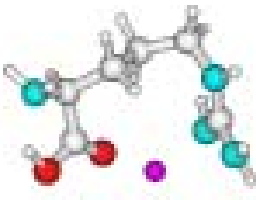

CS_C013

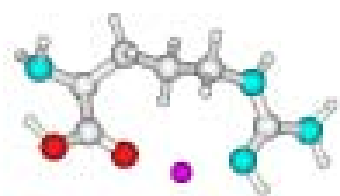

CS_C025

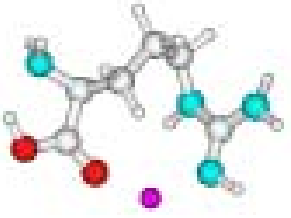

CS_C040

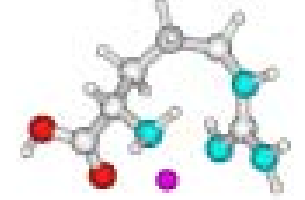

CS_004

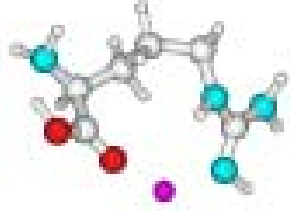

CS_C006

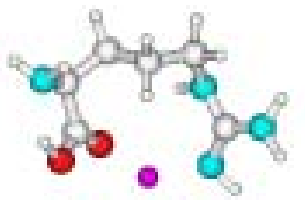

CS_C014

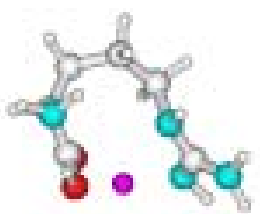

CS_C026

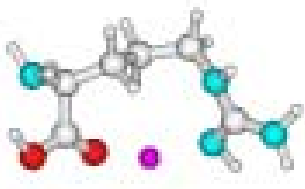

CS_C043

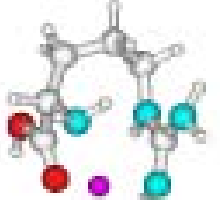

CS_016

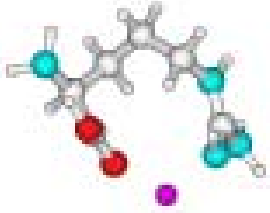

CS_C007

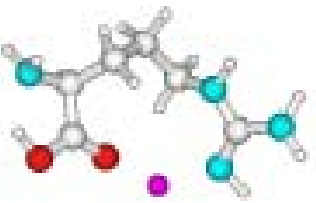

CS_C016

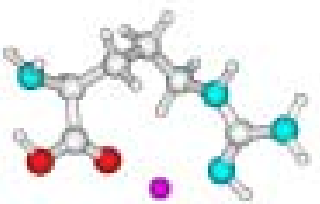

CS_C027

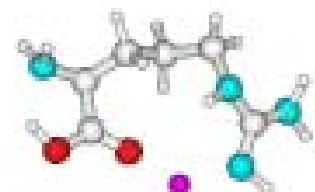

CS $\mathrm{CO44}$

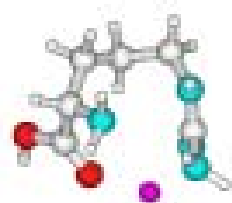

CS_022

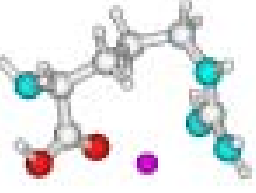

CS_C008

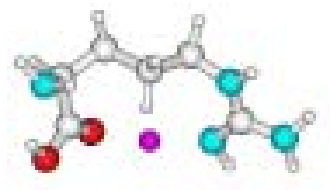

CS_C017

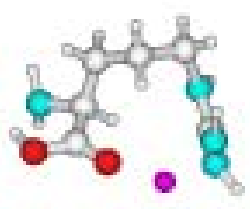

CS_C031

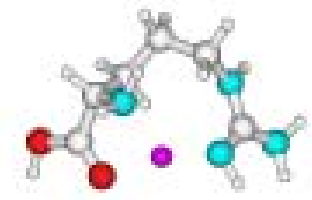

CS_Chs1

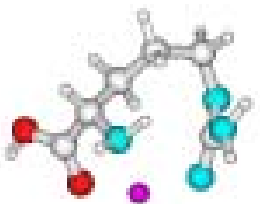

CS_043

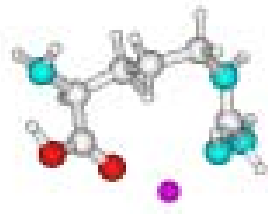

CS_C009

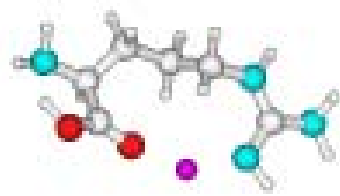

CS_C018

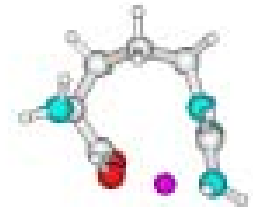

CS_C033

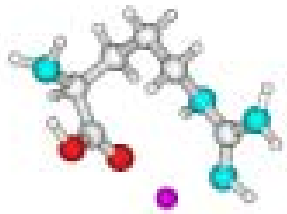

CS_Chs2

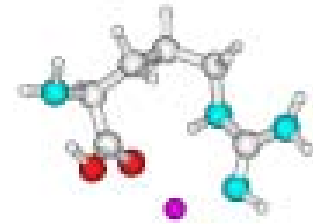

CS_C001

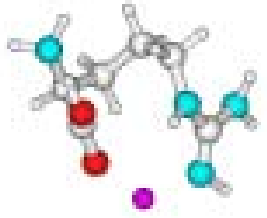

CS_C010

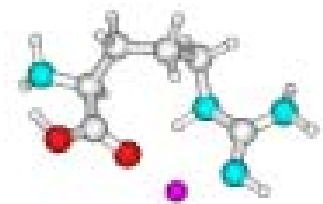

CS_C021

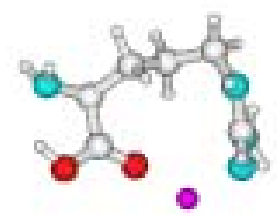

CS_C035

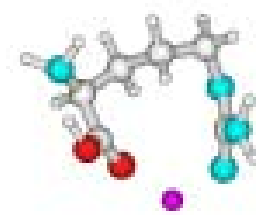

CS_CS1 
Scheme 3b. Geometry optimized conformers of $[\mathrm{Arg}+\mathrm{Li}]^{+}(\mathrm{B} 3 \mathrm{LYP} / 6-31+\mathrm{g}(\mathrm{d}, \mathrm{p}))$.

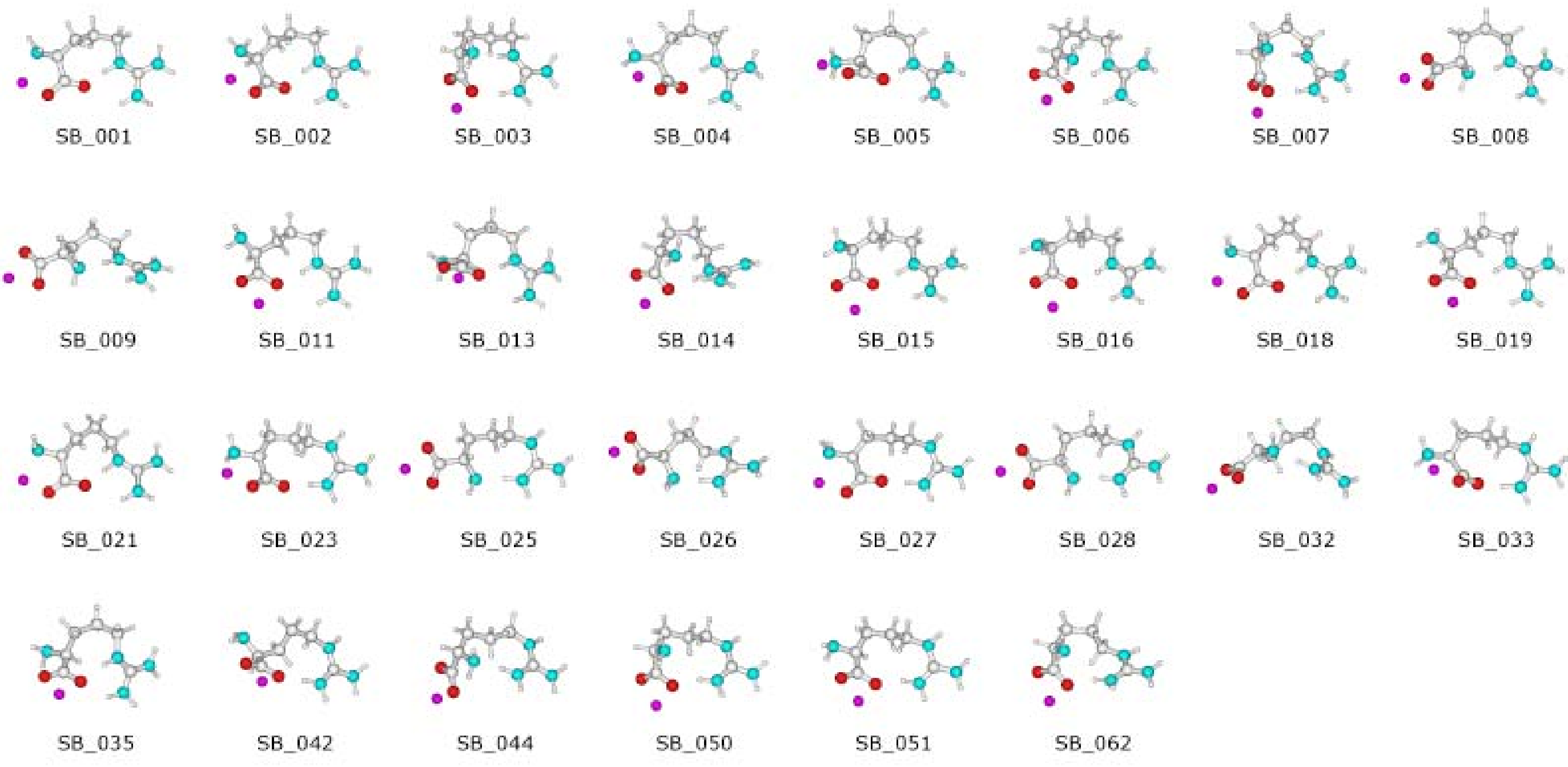


Figure 3. Vibrational spectra for $[A r g+L i]^{+}$conformers from $B 3 L Y P / 6-31+g(d, p)$ calculations. Spectra were constructed using a Gaussian convolution (FWHM=20 $\mathrm{cm}-1$ ) and a scaling factor of 0.98 .
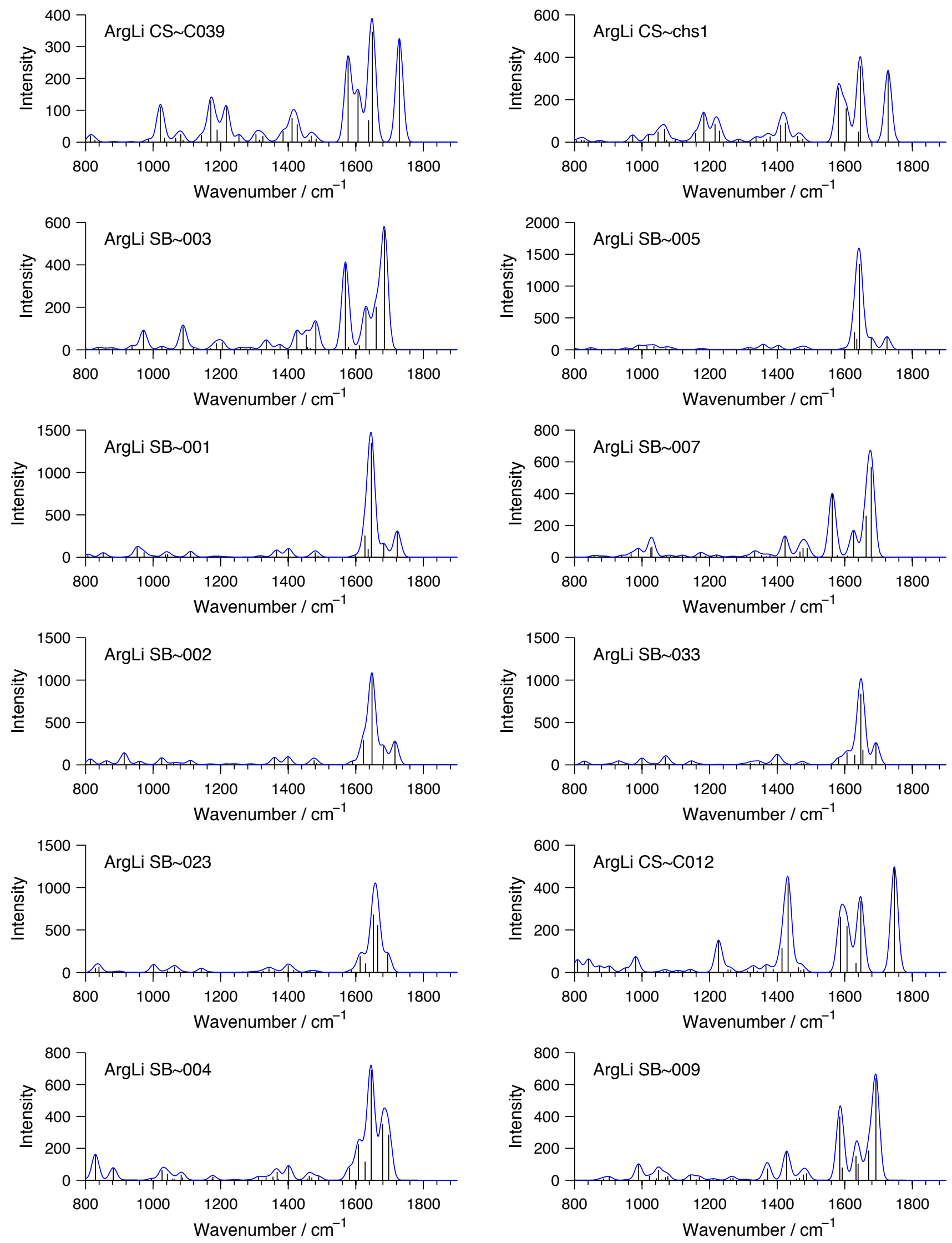
Figure 3 continued.
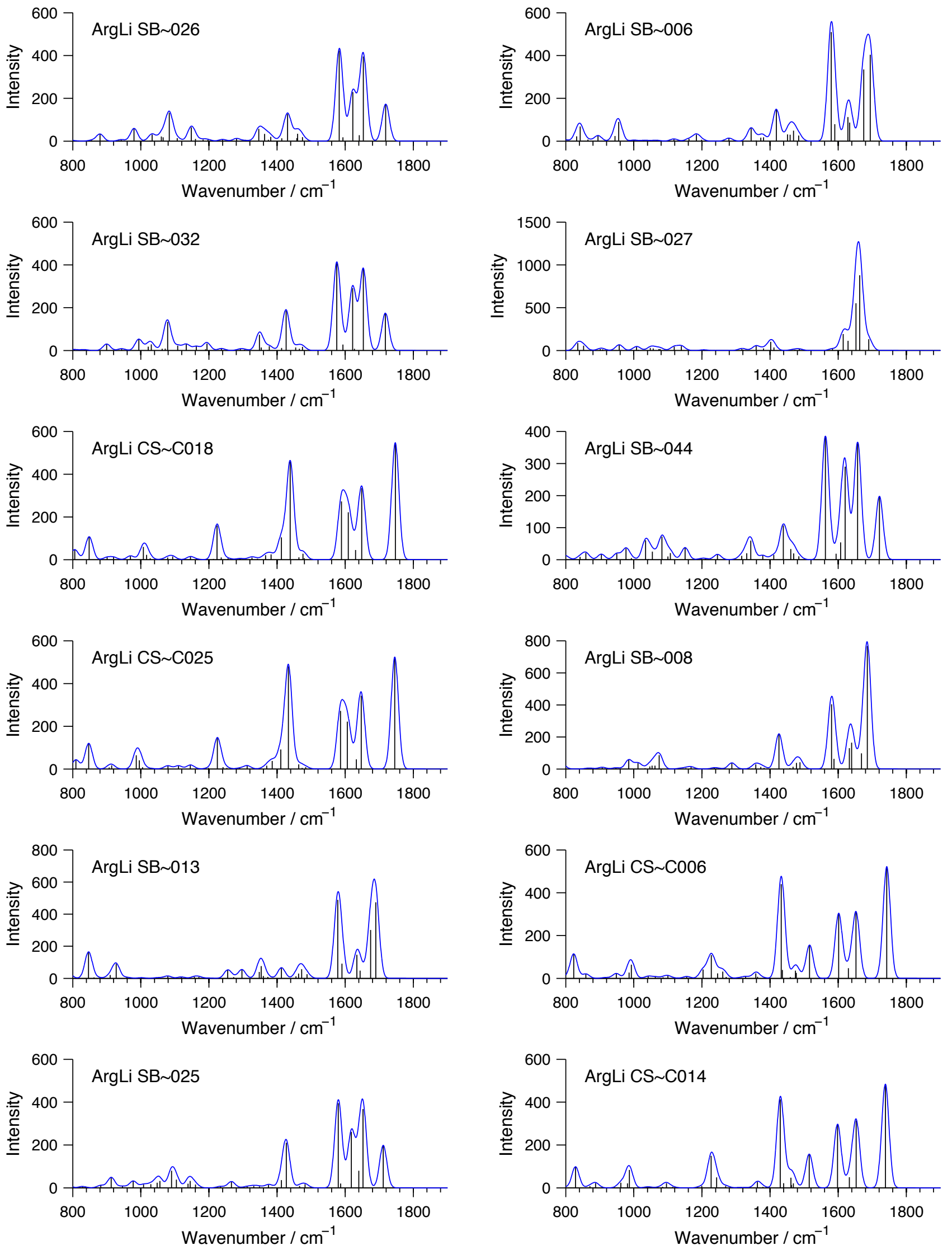
Figure 3 continued.
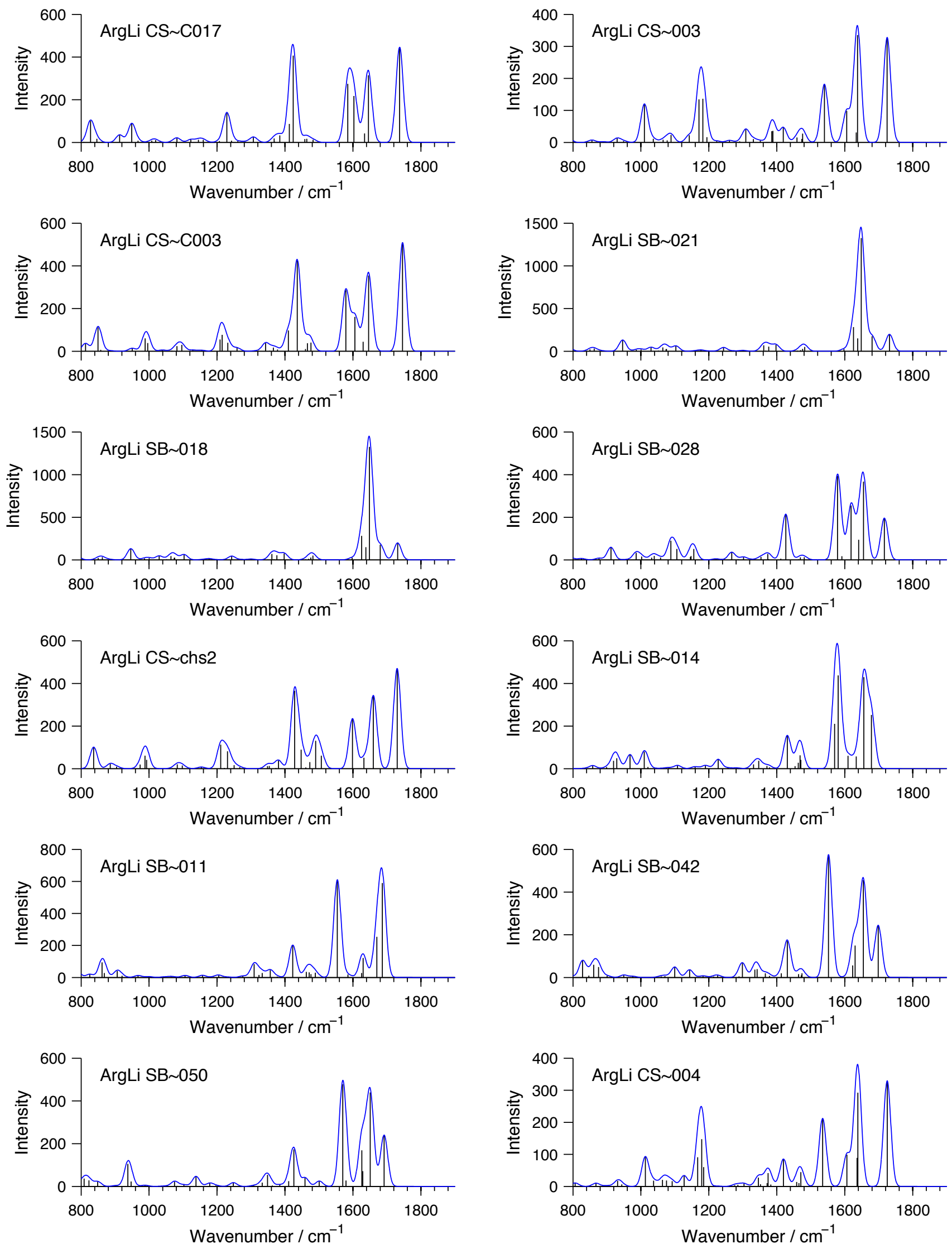
Figure 3 continued.
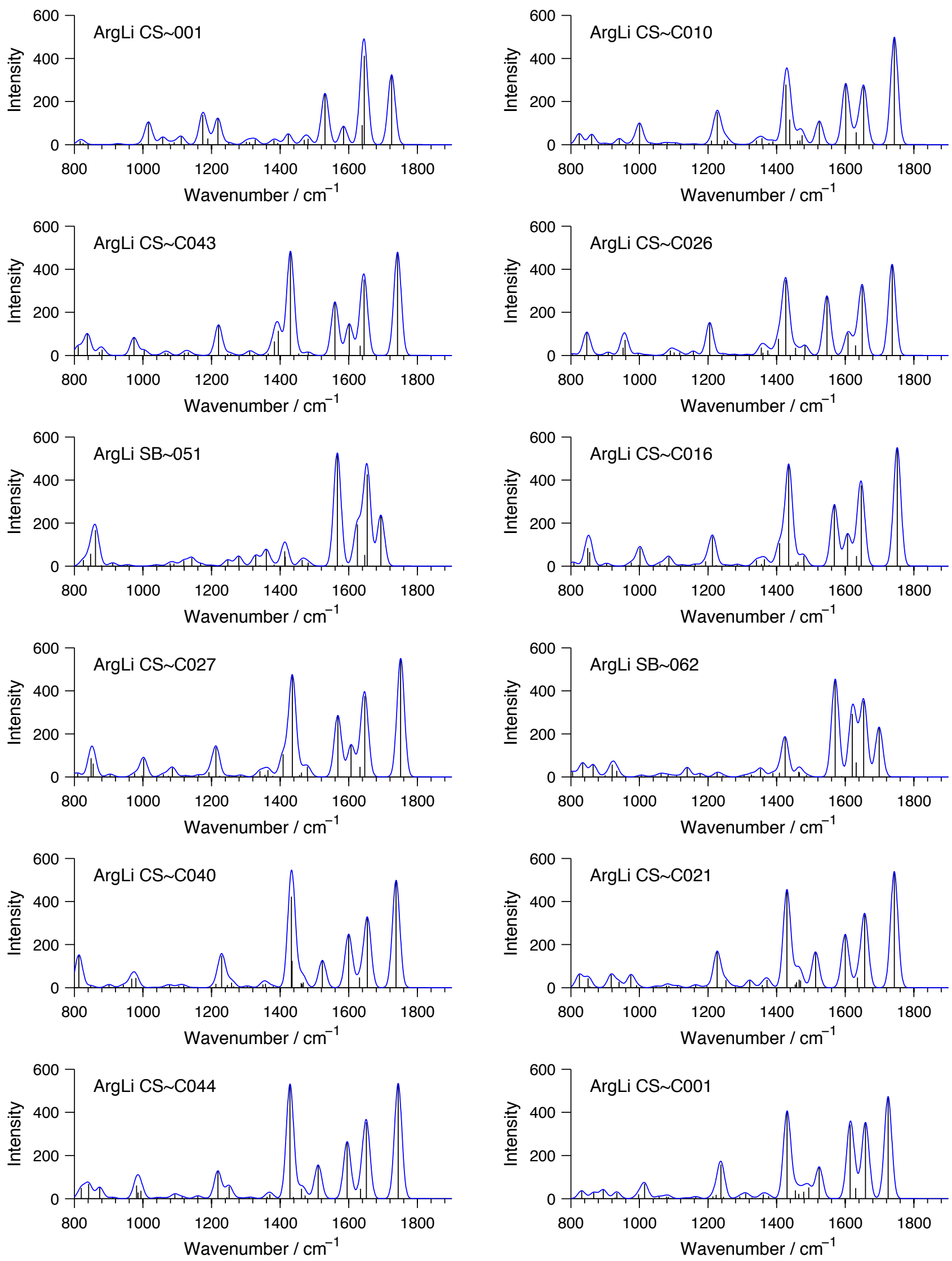
Figure 3 continued.
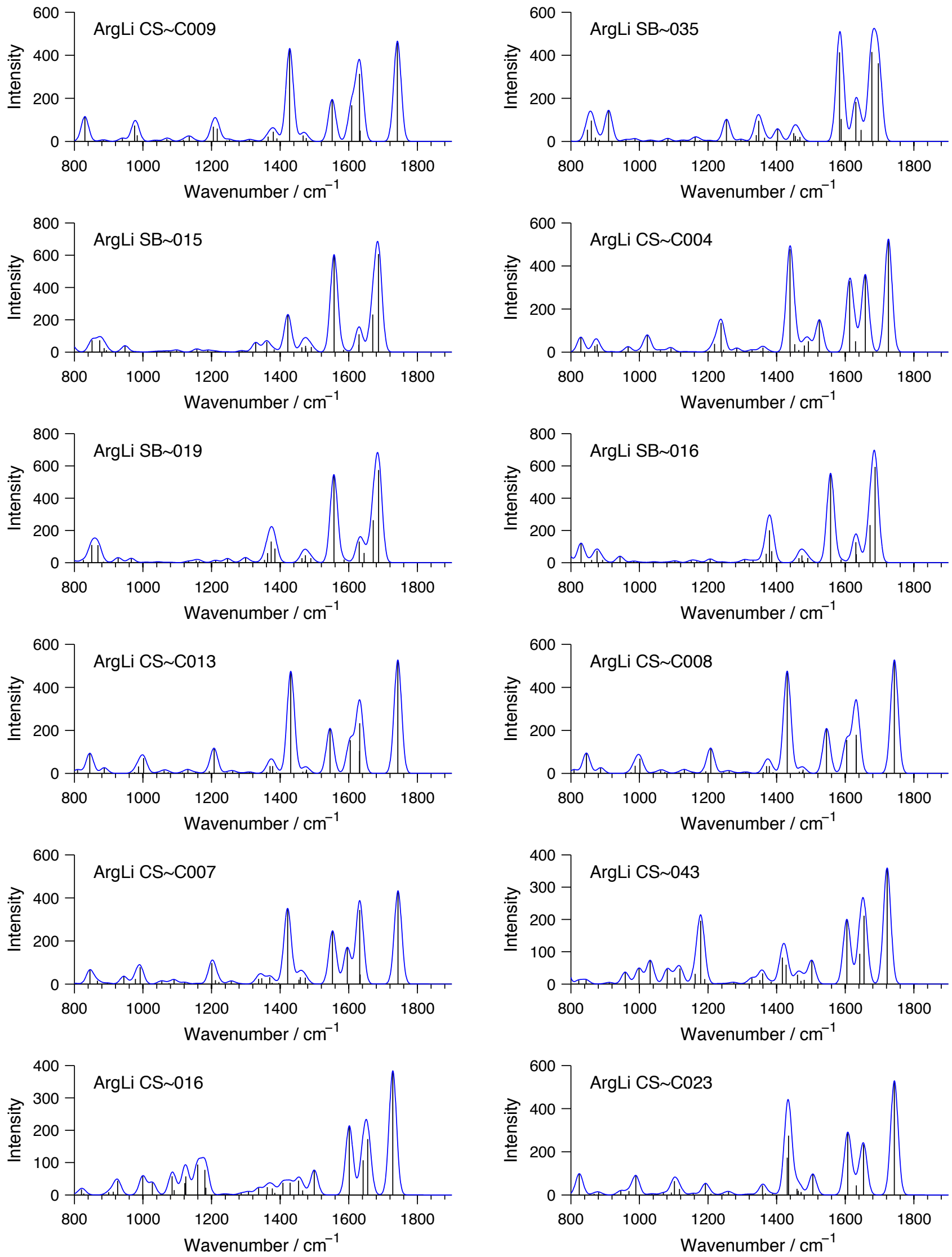
Figure 3 continued.
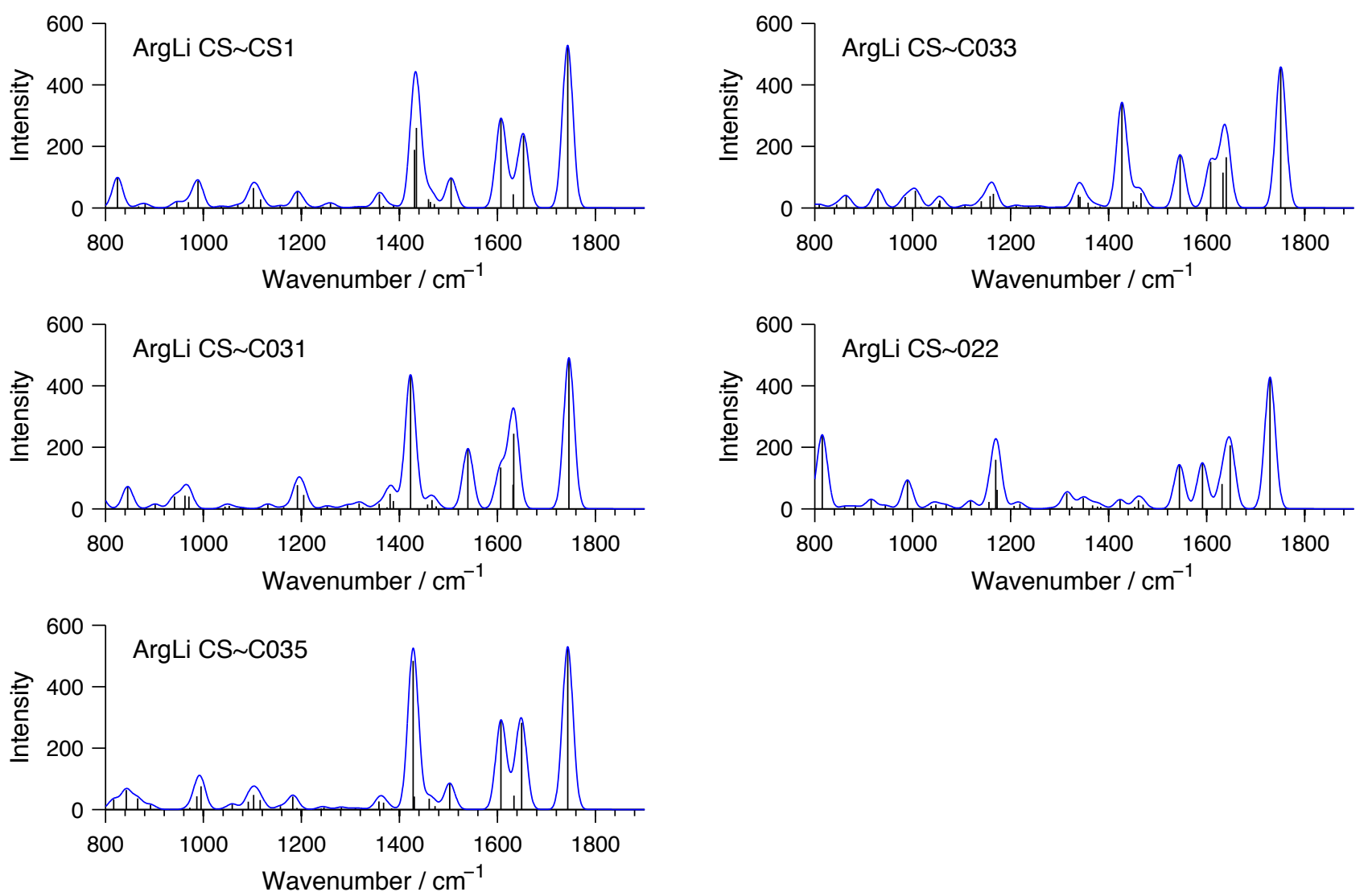
Table 5a. Summary of electronic and thermal energies calculated for MP2-optimized conformers of $[\mathrm{Arg}+\mathrm{Na}]^{+}$

\begin{tabular}{|c|c|c|c|c|c|c|c|c|}
\hline & & & MP2 Opt & & MP2 sp & & & \\
\hline ArgNa MP2 & Name & Converge & $\begin{array}{c}\Delta E_{\mathrm{o}} / \\
\text { kJ mol-1 }\end{array}$ & $\begin{array}{c}\Delta E_{\mathrm{o}} / \\
\text { kJ mol-1 }\end{array}$ & $\begin{array}{c}\Delta \mathrm{H}(298) / \\
\text { kJ mol-1 }\end{array}$ & $\begin{array}{c}\Delta G(298) / \\
\text { kJ mol-1 }\end{array}$ & $\begin{array}{c}\text { MP2 Opt } \\
/ / h\end{array}$ & $\begin{array}{c}\text { MP2 sp } \\
/ / h\end{array}$ \\
\hline SB_003 & SB_D & Min & 0.0 & 0.0 & 0.0 & 0.0 & -766.6066 & -766.9802 \\
\hline SB_007 & SB_D' & Min & 7.0 & 6.1 & 6.2 & 5.7 & -766.6040 & -766.9779 \\
\hline SB_006 & SB_J & Min & 14.5 & 12.8 & 15.3 & 7.7 & -766.6000 & -766.9742 \\
\hline SB_026 & SB_F' & Min & 18.9 & 16.0 & 16.5 & 13.0 & -766.5990 & -766.9737 \\
\hline SB_032 & SB_F' & Min & 20.1 & 17.8 & 18.3 & 14.6 & -766.5983 & -766.9727 \\
\hline CS_C039 & CS_A & Min & 19.0 & 17.9 & 18.4 & 17.2 & -766.5997 & -766.9737 \\
\hline SB_009 & SB_E & Min & 22.8 & 20.8 & 21.3 & 18.1 & -766.5981 & -766.9724 \\
\hline CS_chs1 & & Min & 29.1 & 28.3 & 28.7 & 27.6 & -766.5960 & -766.9699 \\
\hline CS_C012 & CS_B & Min & 37.0 & 29.9 & 31.1 & 25.8 & -766.5923 & -766.9685 \\
\hline CS_C006 & & Min & 37.6 & 32.1 & 33.0 & 27.7 & -766.5921 & -766.9678 \\
\hline CS_C009 & CS_L & Min & 34.8 & 32.4 & 32.8 & 29.6 & -766.5935 & -766.9680 \\
\hline
\end{tabular}

\begin{tabular}{|ll|}
\hline Geometry Optimization & MP2/6-31+g(d,p) \\
Thermal Corrections & B3LYP/6-31+g(d,p) \\
MP2 Single-point & $M P 2 / 6-311++g(2 d, 2 p) / / M P 2 / 6-31+g(d, p)$ \\
\hline
\end{tabular}


Table 5b. Summary of electronic and thermal energies calculated for [Arg+Na] $]^{+}$

\begin{tabular}{|c|c|c|c|c|c|c|c|c|}
\hline & & & B3LYP & & MP2 & & & \\
\hline ArgNa & Name & Converge & $\begin{array}{c}\Delta \mathrm{E}_{\mathrm{o}} / \\
\mathrm{kJ} \mathrm{mol}-1\end{array}$ & $\begin{array}{c}\Delta \mathrm{E}_{\mathrm{o}} / \\
\text { kJ mol-1 }\end{array}$ & $\begin{array}{c}\Delta H(298) / \\
\text { kJ mol-1 }\end{array}$ & $\begin{array}{c}\Delta G(298) / \\
\text { kJ mol-1 }\end{array}$ & $\begin{array}{c}\text { B3LYP E / } \\
h\end{array}$ & $\underset{h}{\text { MP2 E / }}$ \\
\hline SB_003 & SB_D & Min & 0.0 & 0.0 & 0.0 & 0.0 & -768.7874 & -766.9783 \\
\hline SB_007 & & Min & 9.8 & 8.2 & 8.3 & 8.3 & -768.7837 & -766.9752 \\
\hline SB_006 & SB_J & Min & 18.7 & 14.8 & 17.3 & 17.3 & -768.7791 & -766.9716 \\
\hline SB_026 & SB_F' & Min & 13.0 & 16.5 & 17.0 & 17.0 & -768.7820 & -766.9716 \\
\hline SB_009 & SB_E & Min & 8.5 & 17.3 & 19.2 & 19.2 & -768.7830 & -766.9706 \\
\hline SB_013 & SB_K & Min & 17.4 & 17.5 & 19.8 & 19.8 & -768.7795 & -766.9704 \\
\hline SB_005 & & TS & 14.9 & 17.6 & 17.6 & 17.6 & -768.7800 & -766.9699 \\
\hline SB_032 & SB_F & Min & 10.7 & 18.0 & 18.4 & 18.4 & -768.7826 & -766.9708 \\
\hline SB 048 & & Min & 21.2 & 20.5 & 21.2 & 21.2 & -768.7792 & -766.9704 \\
\hline SB 002 & SB G' & Min & 16.1 & 20.9 & 22.1 & 22.1 & -768.7804 & -766.9696 \\
\hline SB 012 & & Min & 16.3 & 21.0 & 22.2 & 22.2 & -768.7804 & -766.9696 \\
\hline SB 044 & & Min & 19.3 & 21.0 & 21.9 & 21.9 & -768.7796 & -766.9699 \\
\hline SB 001 & SB_G & Min & 13.7 & 21.4 & 22.8 & 22.8 & -768.7813 & -766.9693 \\
\hline SB 008 & & Min & 16.0 & 21.8 & 23.5 & 23.5 & -768.7805 & -766.9692 \\
\hline SB_033 & SB I' & Min & 21.7 & 22.8 & 23.1 & 23.1 & -768.7788 & -766.9694 \\
\hline SB_025 & & Min & 17.4 & 23.7 & 24.5 & 24.5 & -768.7805 & -766.9690 \\
\hline SB_023 & & Min & 22.7 & 24.5 & 25.4 & 25.4 & -768.7783 & -766.9686 \\
\hline SB_014 & & Min & 26.1 & 24.7 & 25.3 & 25.3 & -768.7775 & -766.9690 \\
\hline SB_028 & & Min & 18.3 & 25.2 & 26.8 & 26.8 & -768.7798 & -766.9681 \\
\hline SB_042 & & Min & 24.1 & 25.7 & 27.1 & 27.1 & -768.7772 & -766.9675 \\
\hline SB_022 & & Min & 21.9 & 26.7 & 28.1 & 28.1 & -768.7777 & -766.9668 \\
\hline SB_011 & SB_H & Min & 25.4 & 27.0 & 29.2 & 29.2 & -768.7763 & -766.9667 \\
\hline SB_004 & & Min & 20.8 & 27.1 & 27.8 & 27.8 & -768.7794 & -766.9679 \\
\hline SB_050 & & Min & 27.3 & 28.7 & 29.7 & 29.7 & -768.7767 & -766.9671 \\
\hline SB_035 & & Min & 28.0 & 29.2 & 31.2 & 31.2 & -768.7762 & -766.9667 \\
\hline SB_062 & & Min & 30.9 & 29.4 & 31.4 & 31.4 & -768.7748 & -766.9663 \\
\hline SB_051 & & Min & 27.5 & 30.8 & 32.2 & 32.2 & -768.7762 & -766.9659 \\
\hline SB_031 & SB_I & Min & 27.3 & 31.0 & 32.1 & 32.1 & -768.7765 & -766.9661 \\
\hline CS C012 & CS B & Min & 21.2 & 31.9 & 33.1 & 33.1 & -768.7790 & -766.9659 \\
\hline SB 015 & & Min & 29.3 & 32.9 & 35.4 & 35.4 & -768.7748 & -766.9644 \\
\hline SB 027 & & Min & 26.9 & 33.1 & 34.0 & 34.0 & -768.7768 & -766.9654 \\
\hline CS_C006 & & Min & 28.0 & 34.4 & 35.3 & 35.3 & -768.7765 & -766.9650 \\
\hline CS_C009 & CS_L & Min & 37.7 & 34.5 & 34.9 & 34.9 & -768.7731 & -766.9653 \\
\hline CS_C014 & & Min & 27.9 & 35.6 & 36.1 & 36.1 & -768.7769 & -766.9649 \\
\hline CS_C011 & & Min & 33.2 & 35.9 & 35.8 & 35.8 & -768.7753 & -766.9652 \\
\hline SB_021 & & Min & 28.4 & 36.4 & 37.9 & 37.9 & -768.7755 & -766.9634 \\
\hline SB_019 & & Min & 33.7 & 36.4 & 39.1 & 39.1 & -768.7731 & -766.9630 \\
\hline SB_016 & & Min & 32.8 & 36.8 & 39.4 & 39.4 & -768.7735 & -766.9629 \\
\hline CS_C025 & & Min & 24.2 & 37.2 & 38.5 & 38.5 & -768.7778 & -766.9638 \\
\hline CS_C018 & & Min & 25.7 & 38.9 & 40.6 & 40.6 & -768.7771 & -766.9630 \\
\hline CS_C008 & & Min & 37.6 & 39.2 & 40.1 & 40.1 & -768.7728 & -766.9631 \\
\hline CS_C003 & & Min & 30.6 & 39.3 & 40.8 & 40.8 & -768.7751 & -766.9627 \\
\hline CS_C010 & & Min & 41.3 & 39.7 & 40.2 & 40.2 & -768.7717 & -766.9632 \\
\hline $\mathrm{CS}=\mathrm{C} 021$ & & Min & 33.6 & 40.1 & 40.3 & 40.3 & -768.7751 & -766.9635 \\
\hline CS_C007 & & Min & 42.2 & 40.3 & 41.2 & 41.2 & -768.7710 & -766.9626 \\
\hline CS_C013 & & Min & 37.7 & 40.7 & 41.6 & 41.6 & -768.7728 & -766.9626 \\
\hline CS C040 & & Min & 38.2 & 41.3 & 41.8 & 41.8 & -768.7731 & -766.9628 \\
\hline $\mathrm{CS} \mathrm{C044}$ & & Min & 32.7 & 42.6 & 43.3 & 43.3 & -768.7750 & -766.9622 \\
\hline CS C023 & & Min & 45.1 & 42.8 & 42.9 & 42.9 & -768.7705 & -766.9623 \\
\hline CS CS1 & & Min & 45.1 & 42.8 & 43.0 & 43.0 & -768.7705 & -766.9623 \\
\hline CS_C004 & & Min & 31.5 & 43.1 & 44.1 & 44.1 & -768.7752 & -766.9617 \\
\hline $\mathrm{CS} \mathrm{C} 031$ & & Min & 46.9 & 43.3 & 43.9 & 43.9 & -768.7695 & -766.9618 \\
\hline CS_C026 & & Min & 41.9 & 43.4 & 44.3 & 44.3 & -768.7712 & -766.9616 \\
\hline CS_C016 & & Min & 33.8 & 44.2 & 45.6 & 45.6 & -768.7741 & -766.9610 \\
\hline CS_C043 & & Min & 34.1 & 45.3 & 46.2 & 46.2 & -768.7741 & -766.9608 \\
\hline CS_C027 & & Min & 33.3 & 47.3 & 48.7 & 48.7 & -768.7741 & -766.9597 \\
\hline CS_C017 & & Min & 31.5 & 47.7 & 49.2 & 49.2 & -768.7747 & -766.9595 \\
\hline CS_Chs2 & & Min & 33.2 & 50.0 & 51.4 & 51.4 & -768.7753 & -766.9652 \\
\hline CS_001 & & Min & 51.0 & 50.0 & 51.4 & 51.4 & -768.7679 & -766.9592 \\
\hline CS_C035 & & Min & 49.8 & 50.3 & 50.7 & 50.7 & -768.7686 & -766.9593 \\
\hline CS_C001 & & Min & 30.3 & 50.8 & 51.4 & 51.4 & -768.7756 & -766.9588 \\
\hline CS 036 & & Min & 51.7 & 53.2 & 54.4 & 54.4 & -768.7673 & -766.9577 \\
\hline
\end{tabular}




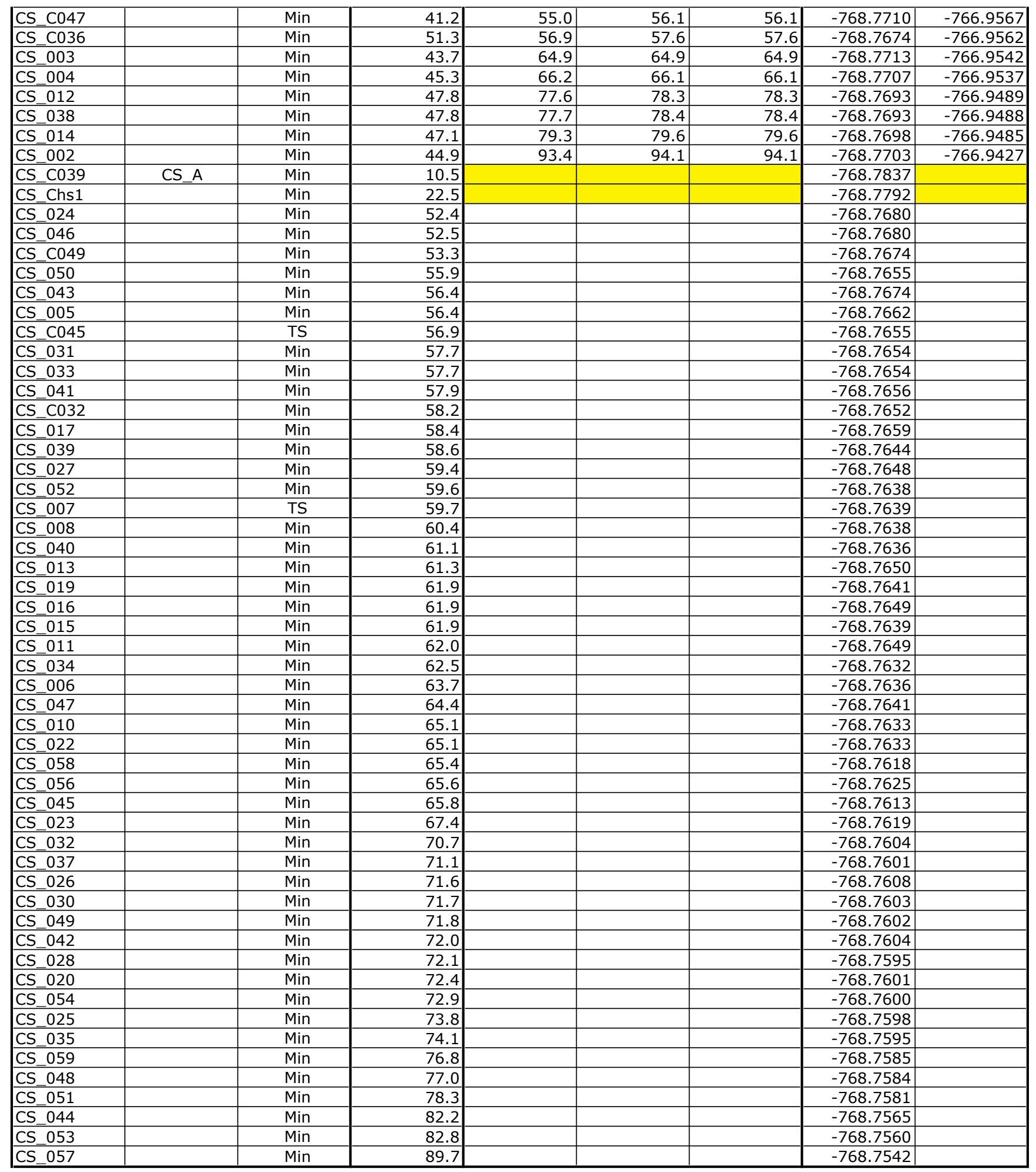

\footnotetext{
Geometry Optimization

Thermal Corrections

MP2 Single-point
}

$B 3 L Y P / 6-31+g(d, p)$

$B 3 L Y P / 6-31+g(d, p)$

$M P 2 / 6-311++g(2 d, 2 p) / / B 3 L Y P / 6-31+g(d, p)$

MP2 job failed due to excessive mixing of frozen core and valence orbitals 
Scheme 4a. Geometry optimized conformers of $[\mathrm{Arg}+\mathrm{Na}]^{+}(\mathrm{B} 3 \mathrm{LYP} / 6-31+\mathrm{g}(\mathrm{d}, \mathrm{p}))$.

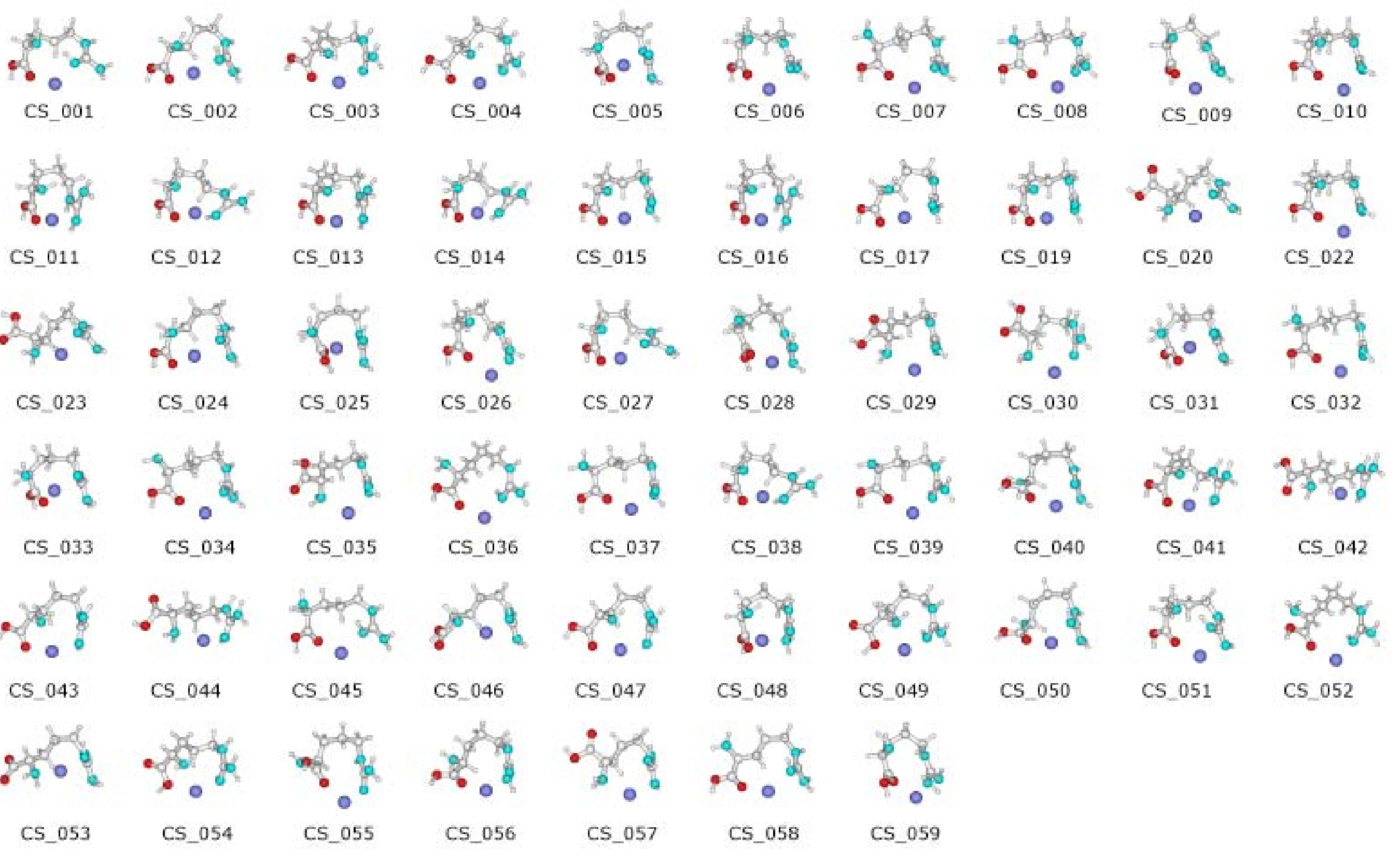


Scheme 4b. Geometry optimized conformers of $[\mathrm{Arg}+\mathrm{Na}]^{+}(\mathrm{B} 3 \mathrm{LYP} / 6-31+\mathrm{g}(\mathrm{d}, \mathrm{p}))$.

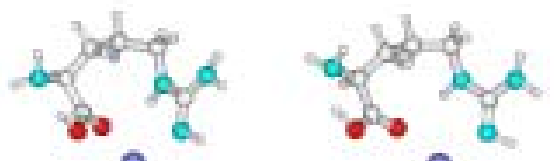

CS_C001

CS $\operatorname{Co10}$

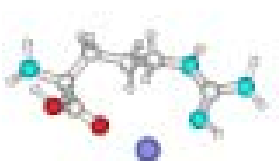

CS_C019
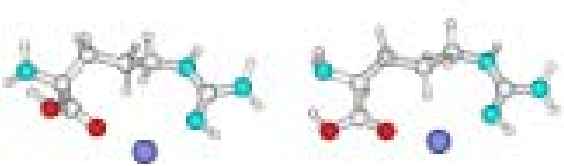

CS_C028

CS C037

- $\int_{0}^{x} e^{2}$

CS $\mathrm{C} 046$
CS_C002

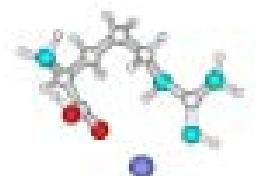

CS_C011

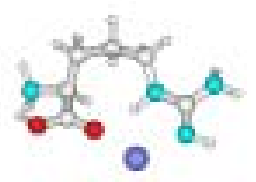

CS_C020

$$
\text { CS_C029 }
$$

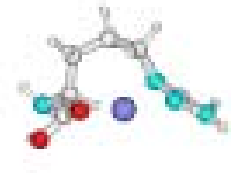

CS_C038

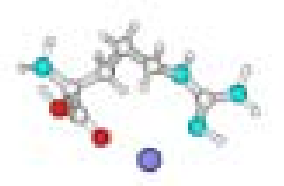

CS_C003

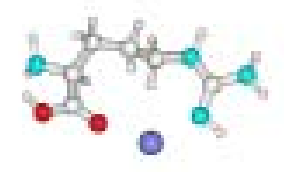

CS C012

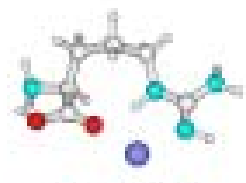

CS_C021

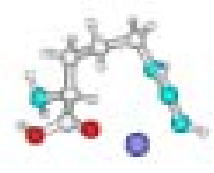

CS_C030

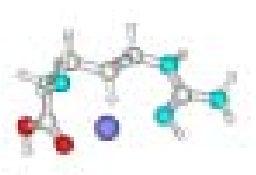

CS_C039

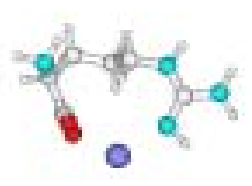

CS_C047

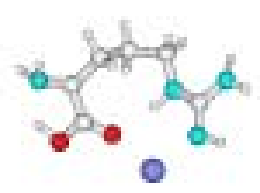

CS_C048
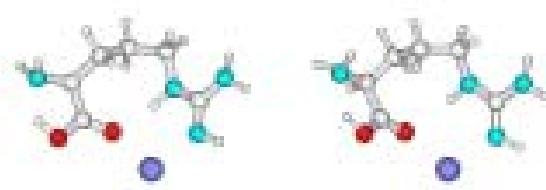

CS_C004

CS_C005

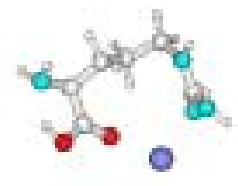

CS $\mathrm{C} 013$

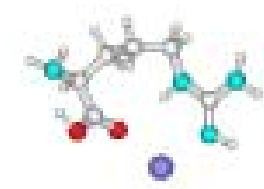

CS_C014

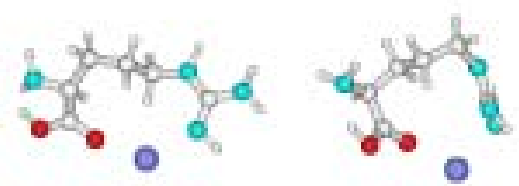

CS_C022

CS_C023

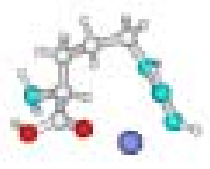

CS_C031

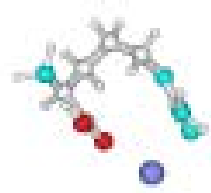

CS_C032
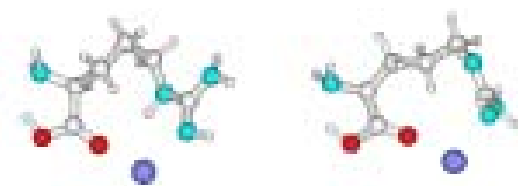

$$
\text { CS_C040 }
$$

CS_C041
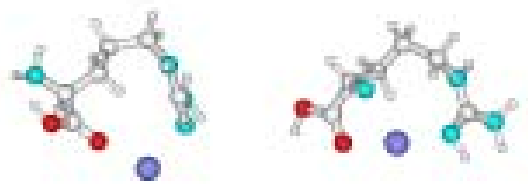

CS $\operatorname{Co49}$

CS_Chs1
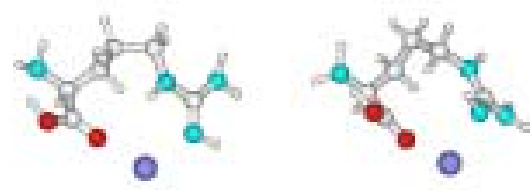

CS_C006

CS_C007
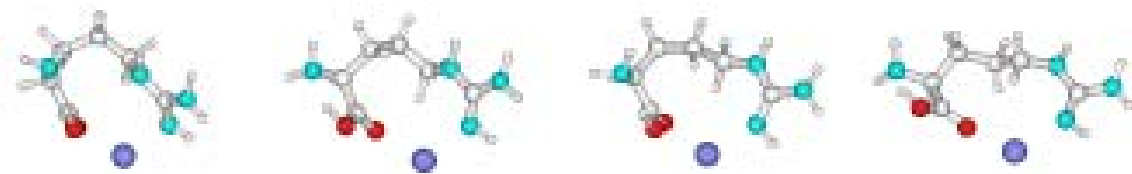

CS $\mathrm{C} 015$

CS C016

CS $\mathrm{C} 017$

CS $\mathrm{C} 018$

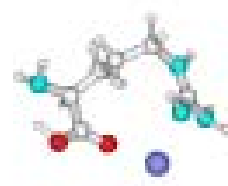

CS_C024
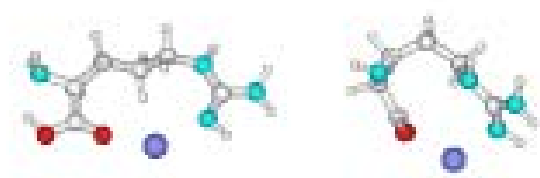

C5_C026

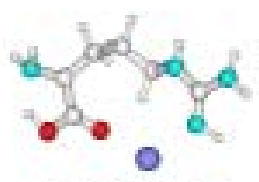

CS_C025

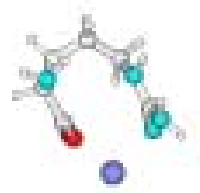

CS_C033

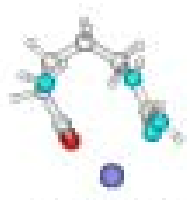

CS_C034
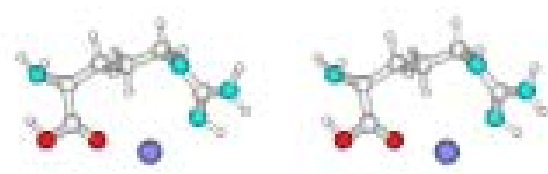

$$
\text { CS_C042 }
$$

CS_C043

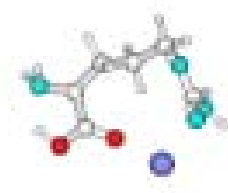

CS_C035

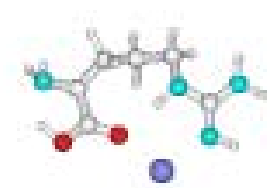

CS_C044
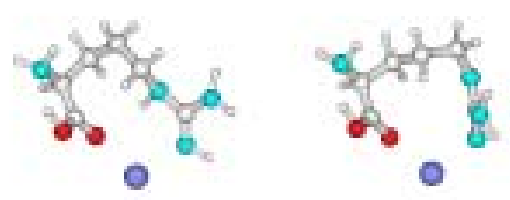

CS_Chs2

CS_CS1
CS_C027
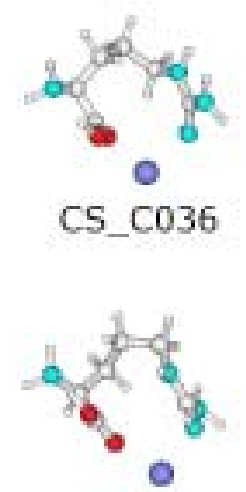

CS_C045 
Scheme 4c. Geometry optimized conformers of $[\mathrm{Arg}+\mathrm{Na}]^{+}(\mathrm{B} 3 \mathrm{LYP} / 6-31+\mathrm{g}(\mathrm{d}, \mathrm{p}))$.

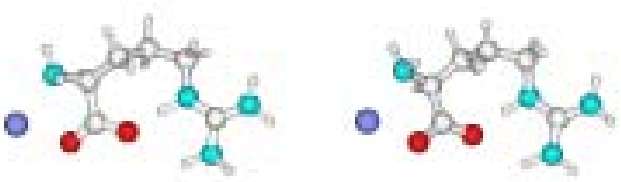

SB_001

SB_002

- ${ }^{0} p^{d} p^{2}$

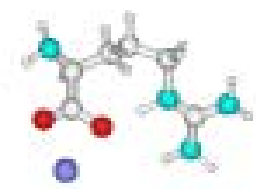

SB_009

SB_010

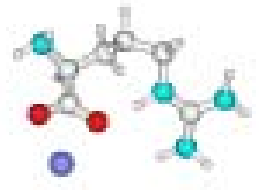

$-0_{0}^{2} \frac{2}{20}$

SB_019

-0 odge

SB_031
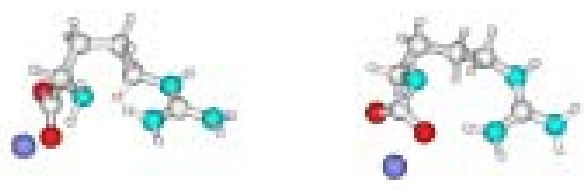

SB_050
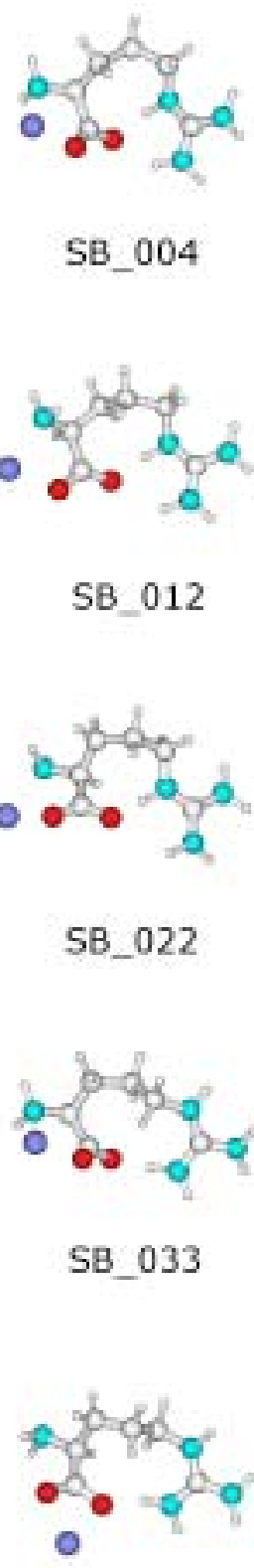

SB_004

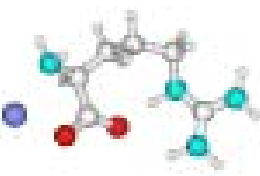

SB_012

- $0^{2}=a^{2}$

SB_022

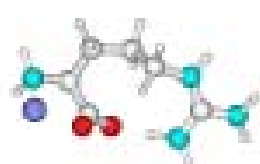

SB_033

SB_051

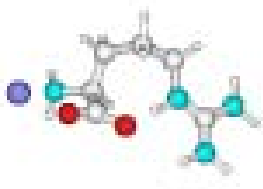

SB_005

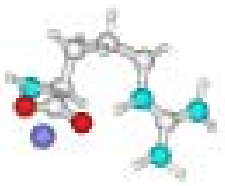

SB 013
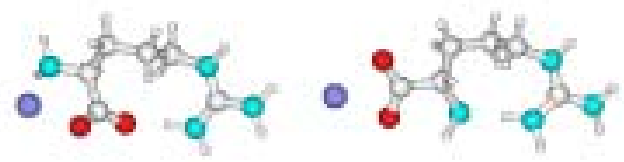

SB_023

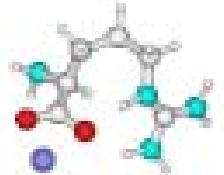

SB_035

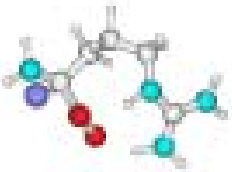

SB_053

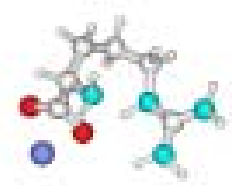

SB_006

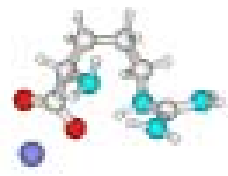

SB 014

SB_025

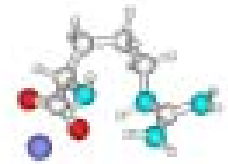

SB_038

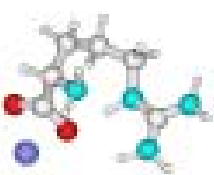

SB 055

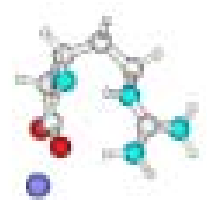

SB_007

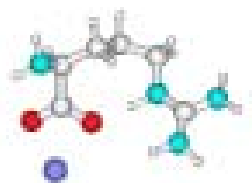

SB 015

- ore bép.

SB_026

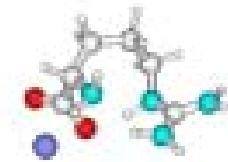

SB_039

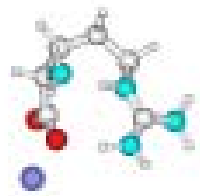

SB 056
- $28 e^{8}$

SB_008

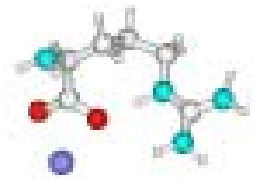

SB 016

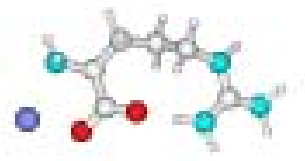

SB_027

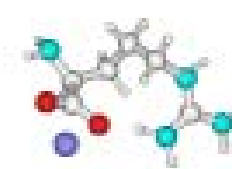

SB_042

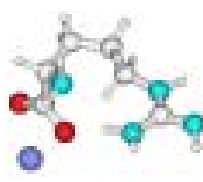

SB_062 
Figure 4. Vibrational spectra for $[\mathrm{Arg}+\mathrm{Na}]^{+}$conformers from B3LYP/6-31+g(d,p) calculations. Spectra were constructed using a Gaussian convolution (FWHM=20 $\mathrm{cm}-1)$ and a scaling factor of 0.98 .
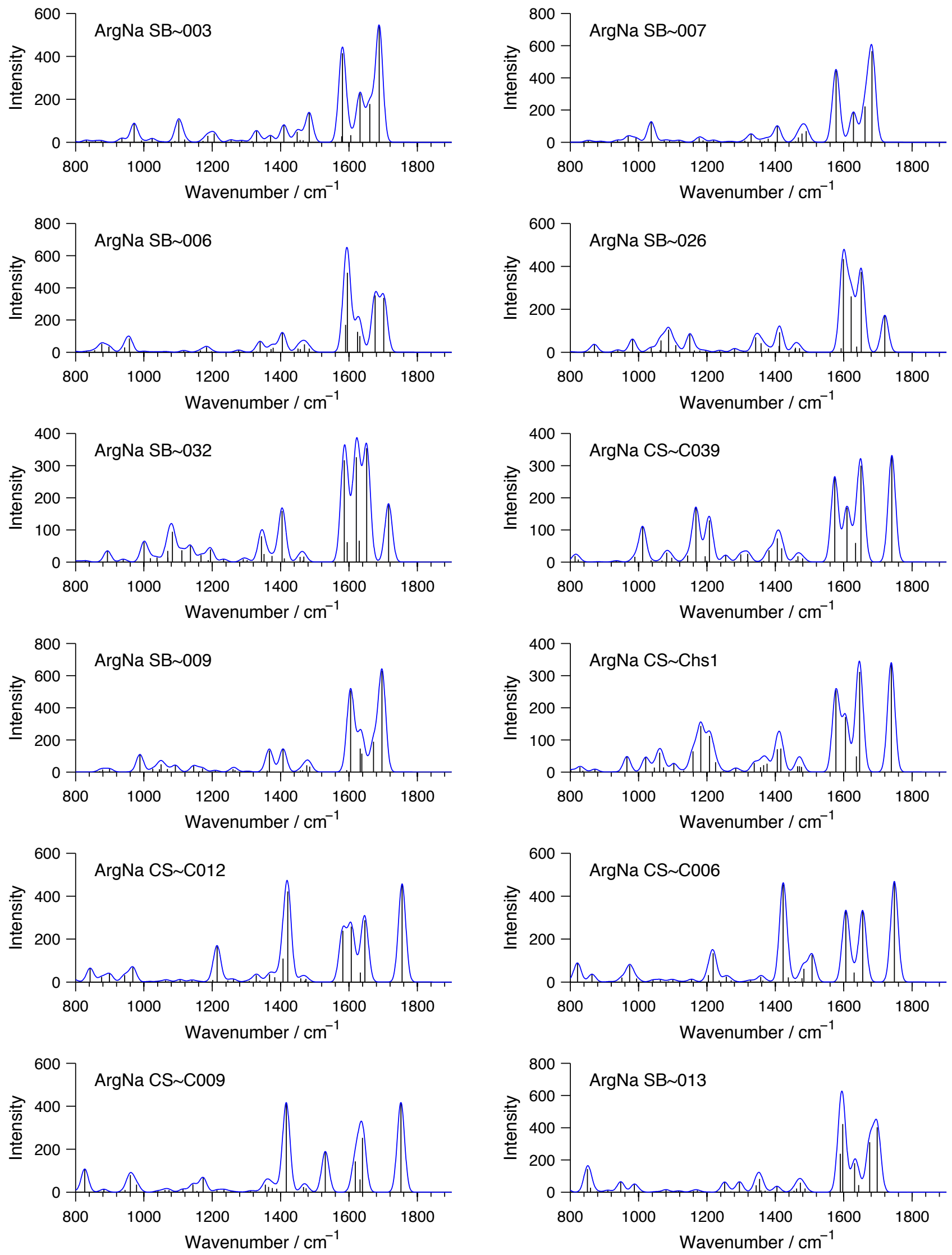
Figure 4 continued.
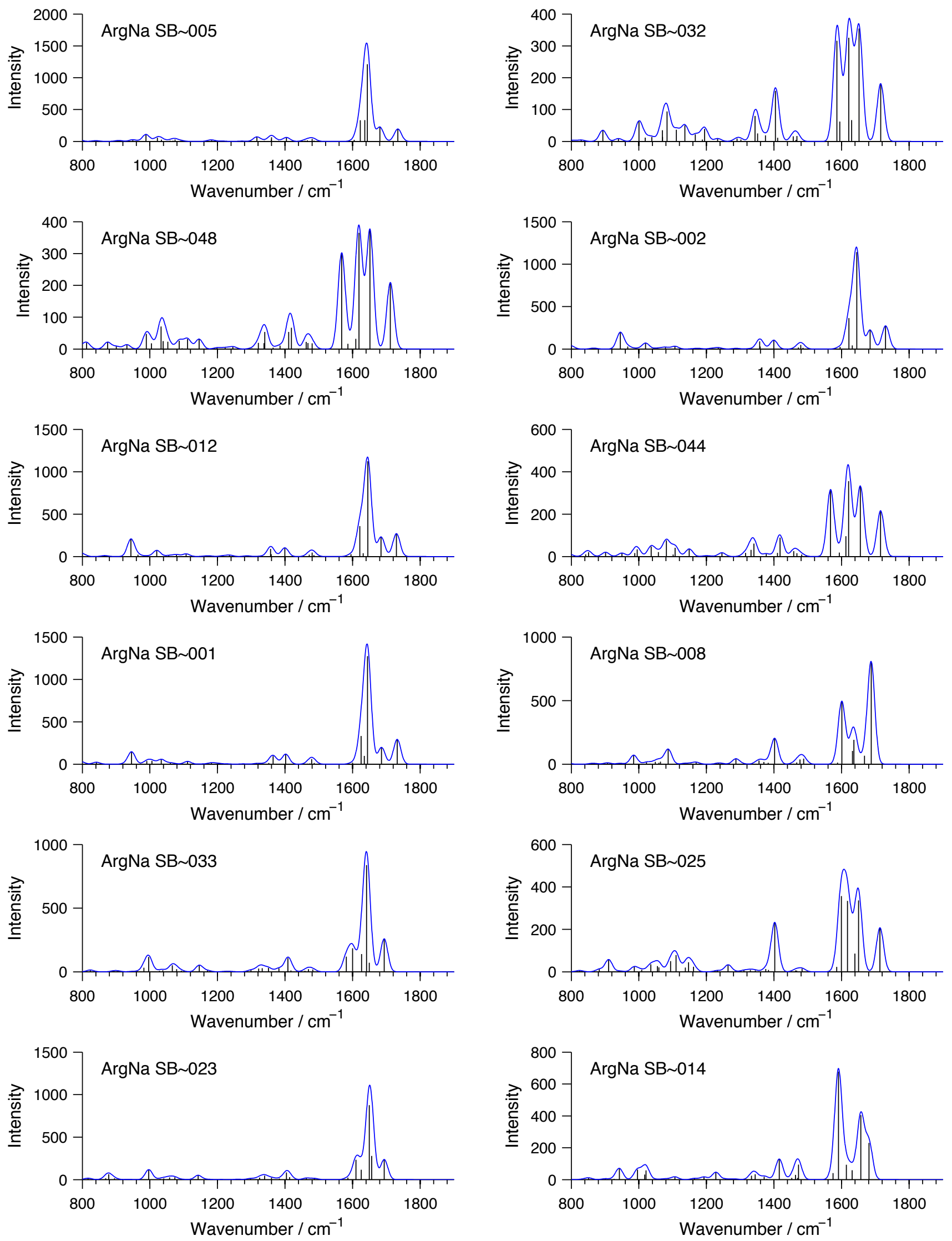
Figure 4 continued.
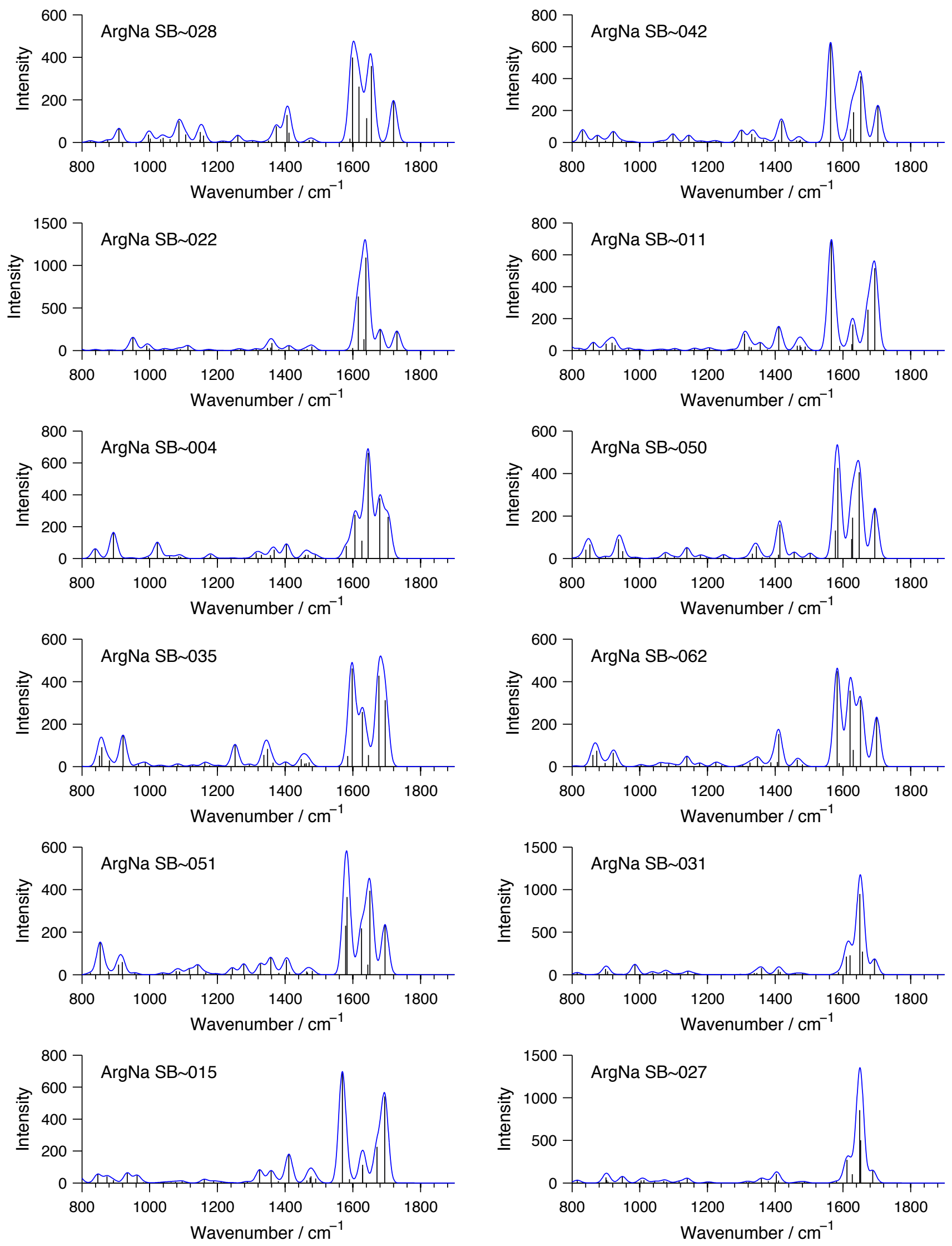
Figure 4 continued.
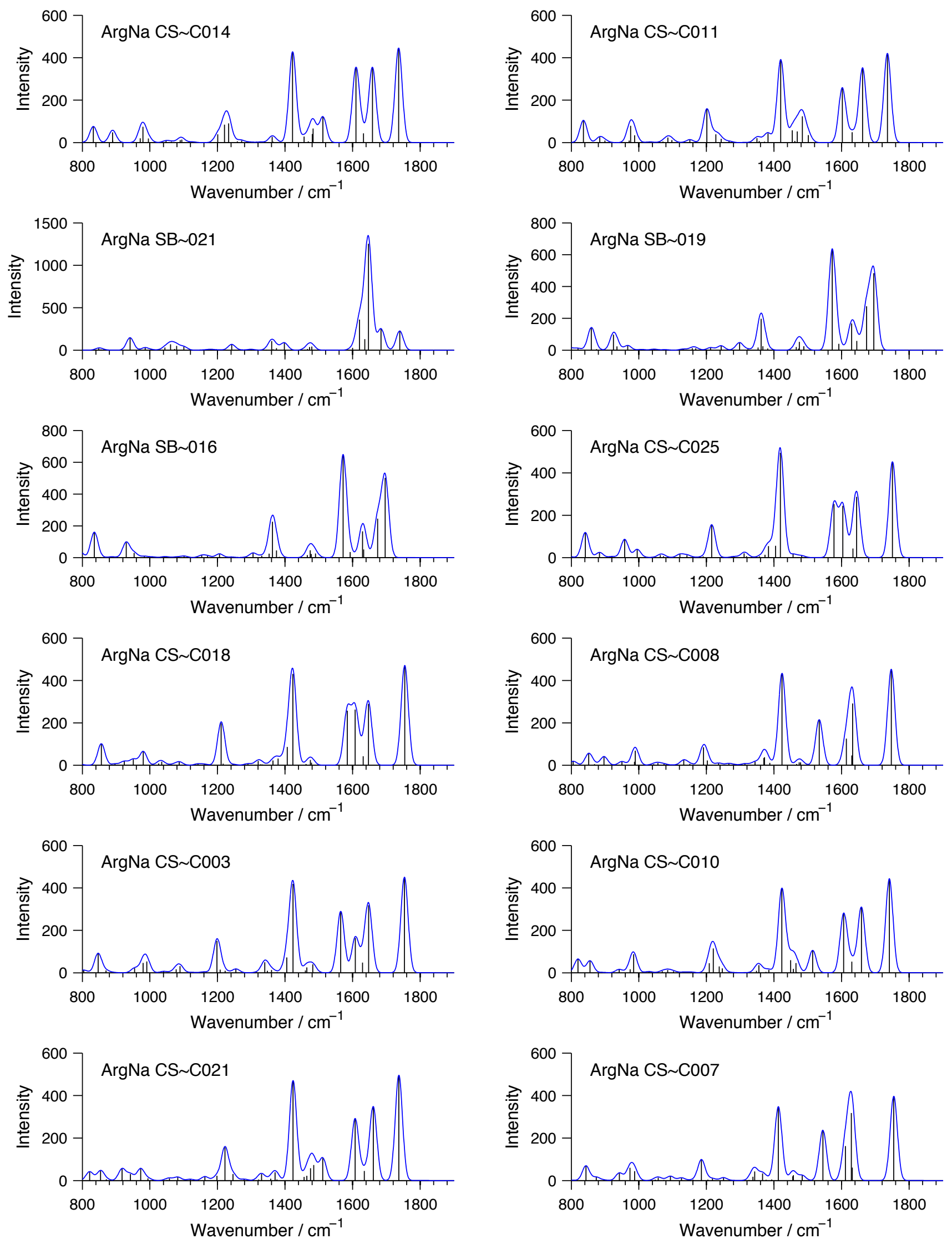
Figure 4 continued.
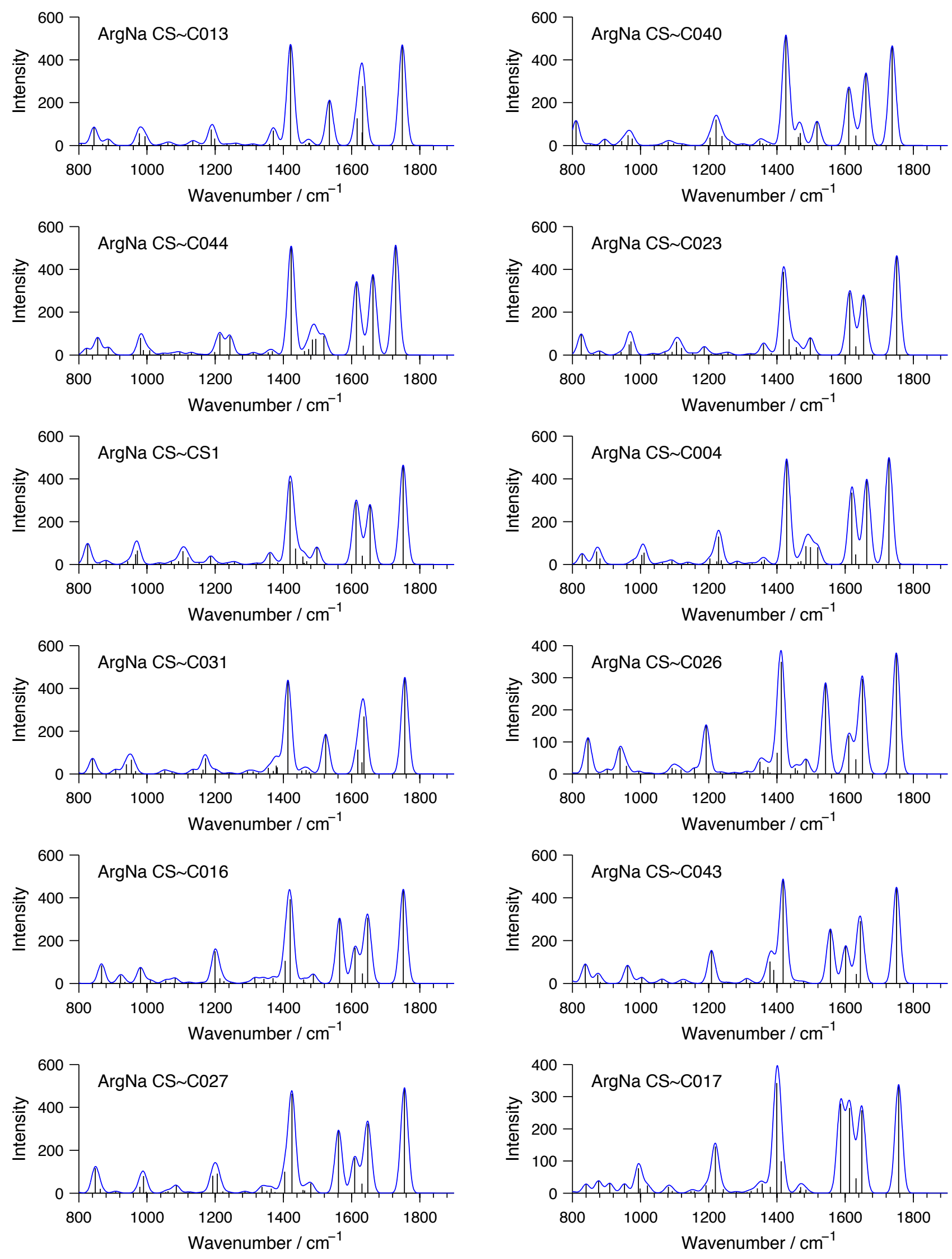
Figure 4 continued.
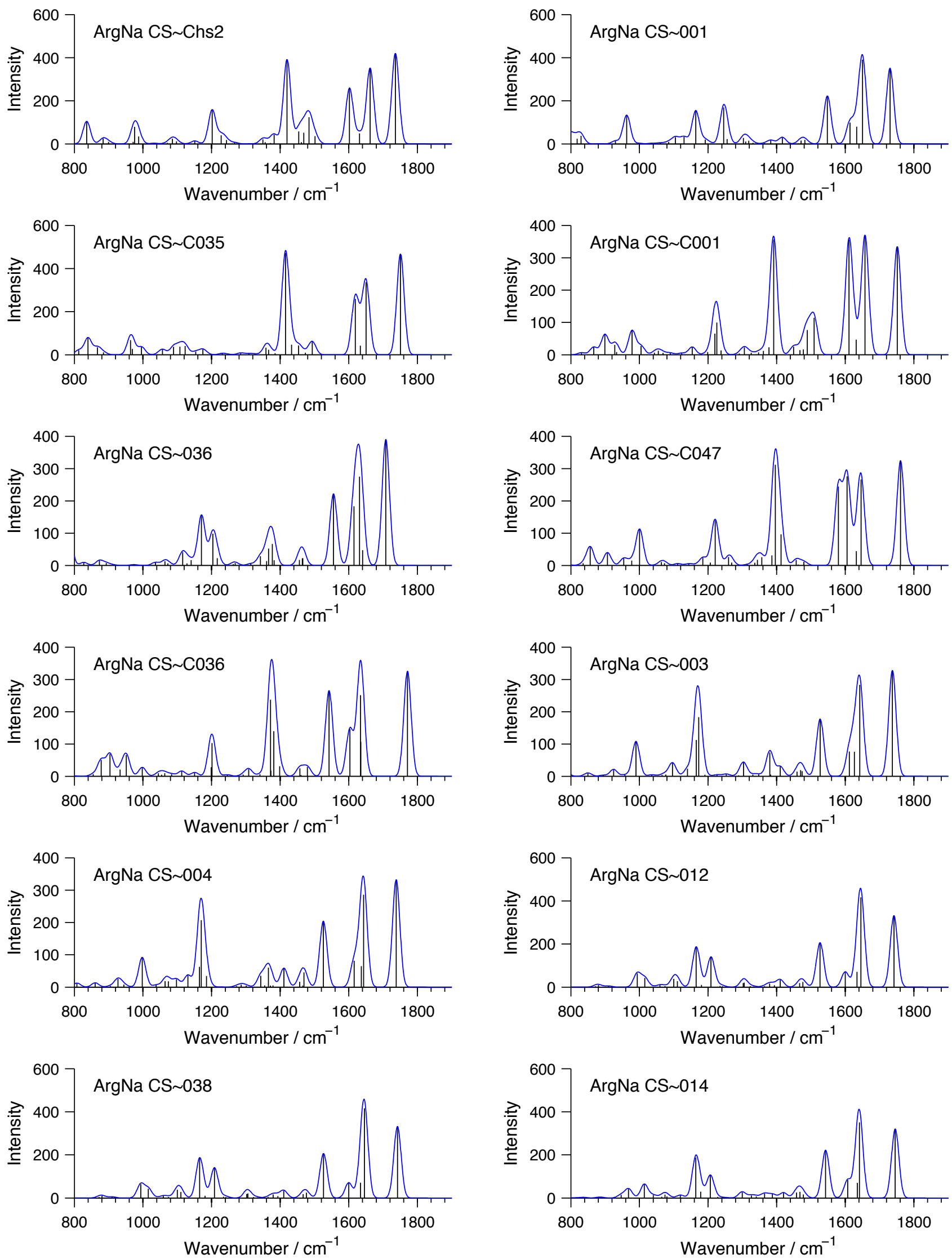
Figure 4 continued.
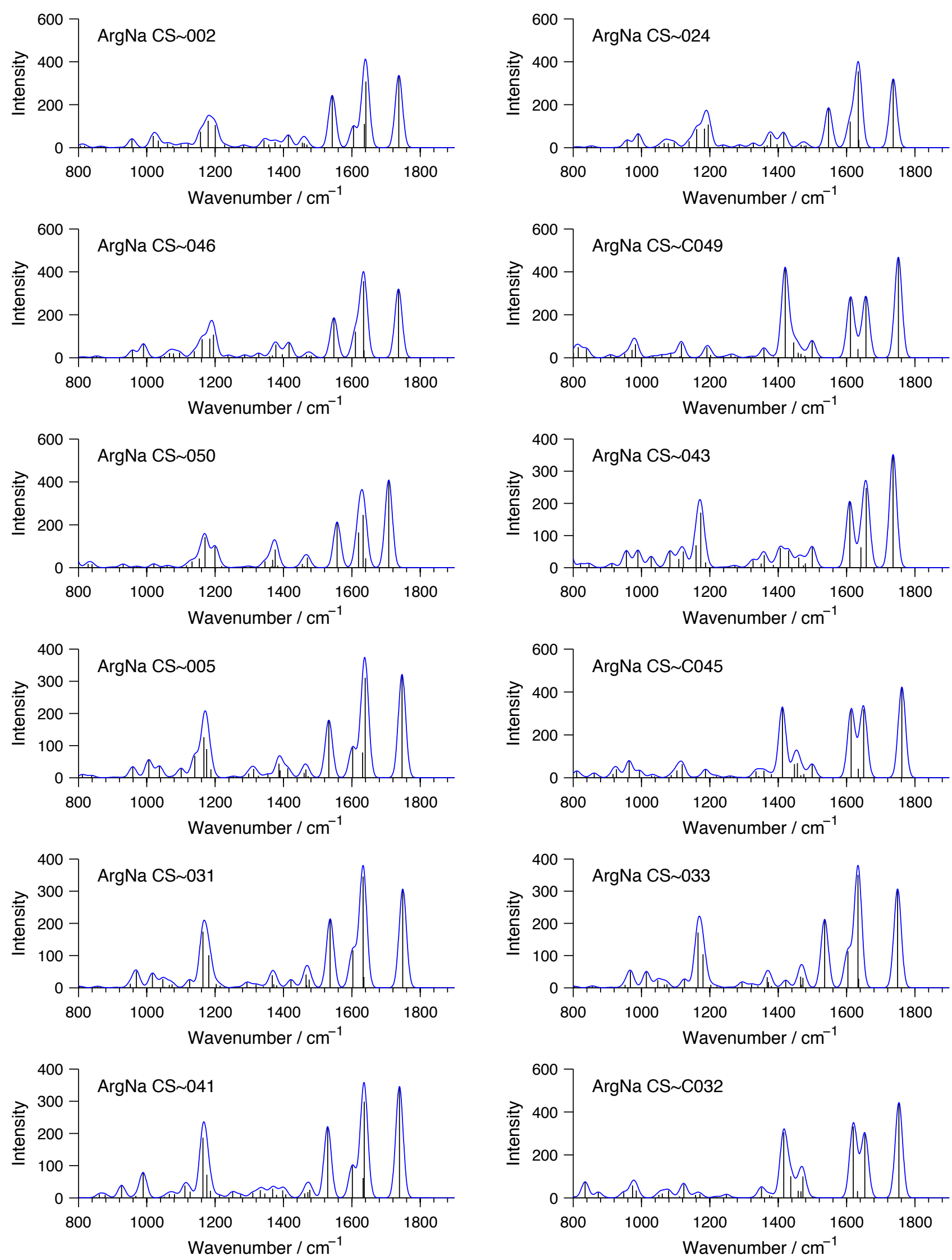
Figure 4 continued.
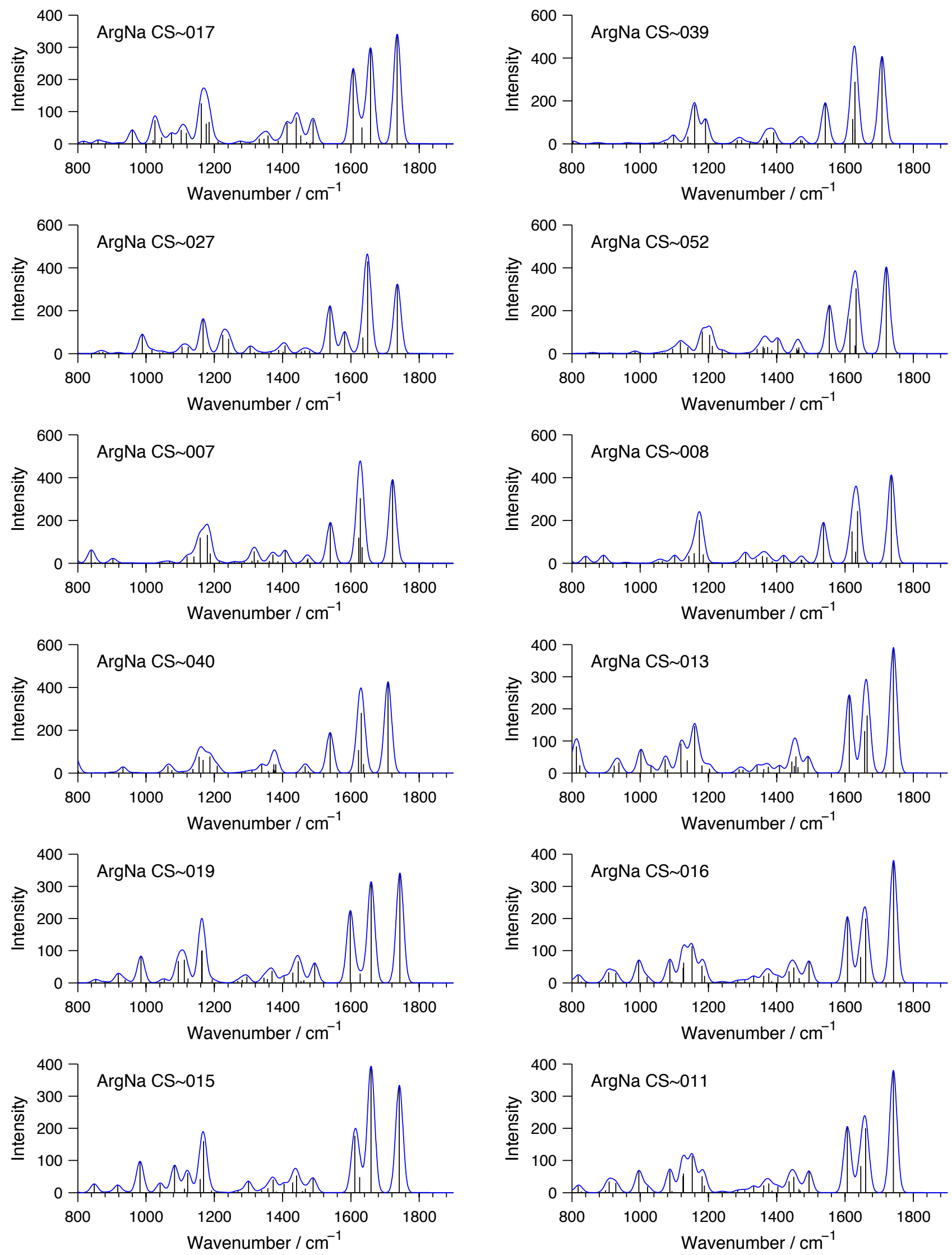
Figure 4 continued.
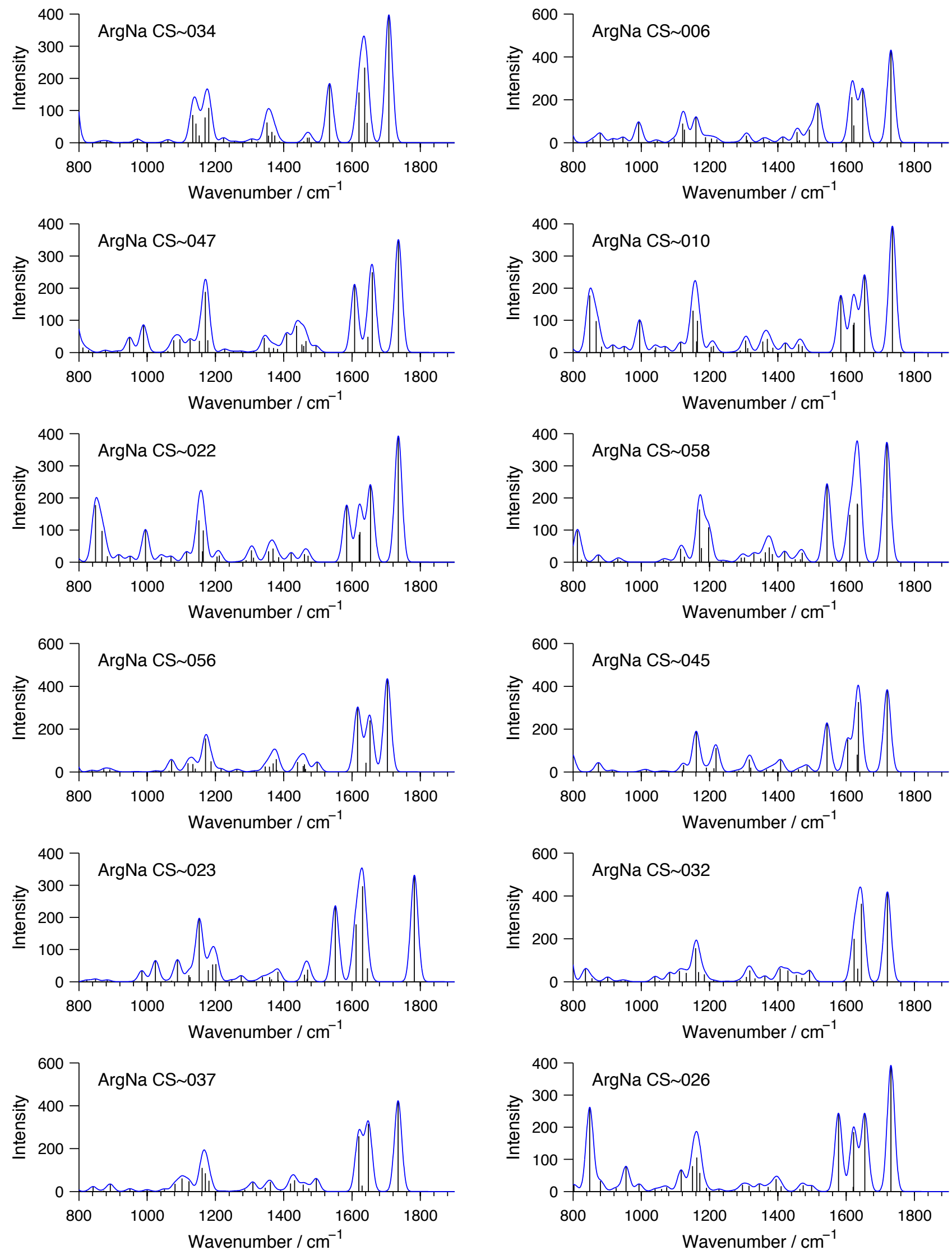
Figure 4 continued.
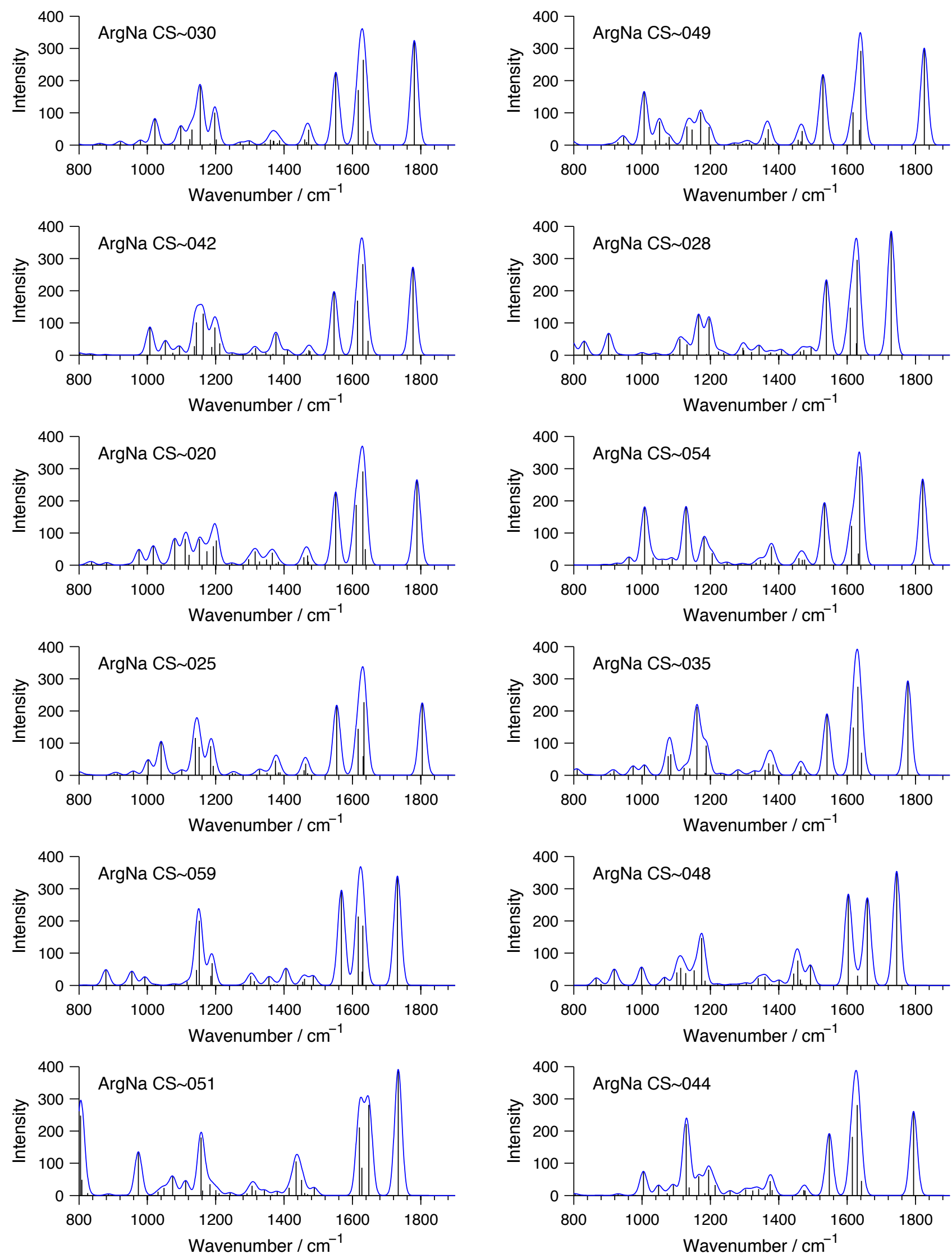
Figure 4 continued.
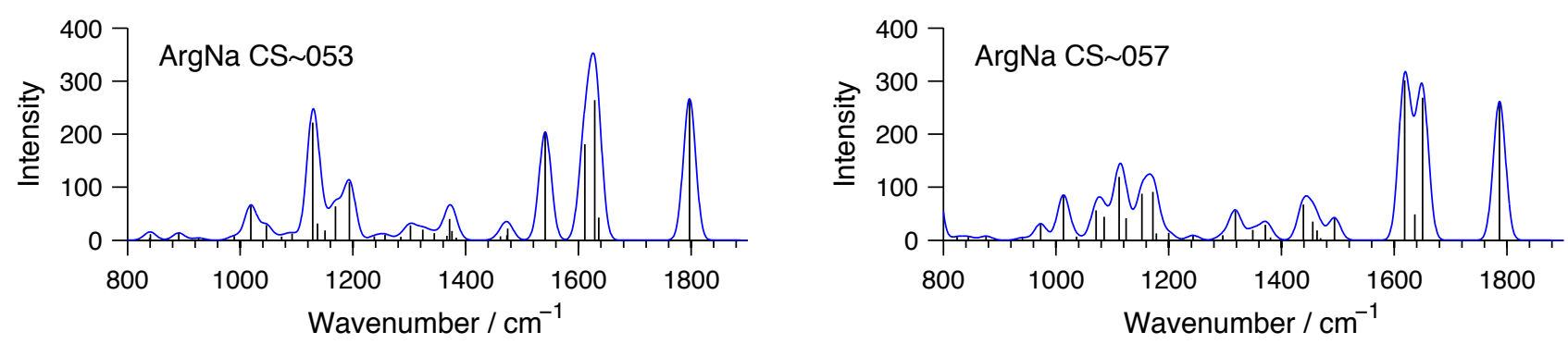
Table 6. Summary of electronic and thermal energies calculated for $[\mathrm{Arg}+\mathrm{K}]^{+}$.

\begin{tabular}{|c|c|c|c|c|c|c|c|c|}
\hline & & & B3LYP & & MP2 & & & \\
\hline ArgK & Name & Converge & $\begin{array}{c}\Delta \mathrm{E}_{\mathrm{o}} / \\
\mathrm{kJ} \mathrm{mol-1}\end{array}$ & $\begin{array}{c}\Delta E_{\mathrm{o}} / \\
\mathrm{kJ} \mathrm{mol-1}\end{array}$ & $\begin{array}{c}\Delta H(298) / \\
\text { kJ mol-1 }\end{array}$ & $\begin{array}{c}\Delta G(298) / \\
k J ~ m o l-1\end{array}$ & $\begin{array}{c}\text { B3LYP E / } \\
h\end{array}$ & $\begin{array}{c}\text { MP2 E / } \\
h\end{array}$ \\
\hline SB 003 & SB_D & Min & 0.0 & 0.0 & 0.0 & 0.0 & -1206.4048 & -1204.4741 \\
\hline SB 007 & & Min & 9.7 & 7.0 & 7.1 & 7.1 & -1206.4011 & -1204.4715 \\
\hline SB_006 & SB_J & Min & 19.3 & 14.7 & 17.1 & 17.1 & -1206.3963 & -1204.4674 \\
\hline SB_013 & SB_K & Min & 17.0 & 16.7 & 19.0 & 18.9 & -1206.3969 & -1204.4663 \\
\hline SB_026 & SB_F' & Min & 15.0 & 18.2 & 18.8 & 18.8 & -1206.3984 & -1204.4665 \\
\hline SB_009 & SB_E & Min & 11.6 & 19.8 & 21.7 & 21.7 & -1206.3990 & -1204.4652 \\
\hline SB_032 & SB_F & Min & 13.4 & 20.6 & 20.9 & 20.9 & -1206.3989 & -1204.4655 \\
\hline SB_005 & & Min & 20.7 & 21.1 & 23.0 & 23.0 & -1206.3950 & -1204.4642 \\
\hline SB_014 & & Min & 26.1 & 24.4 & 25.0 & 25.0 & -1206.3948 & -1204.4648 \\
\hline SB_001 & SB_G & Min & 19.8 & 24.7 & 26.4 & 18.8 & -1206.3961 & -1204.4636 \\
\hline SB_008 & & Min & 20.0 & 24.8 & 26.6 & 26.6 & -1206.3962 & -1204.4637 \\
\hline SB_012 & & Min & 23.9 & 26.2 & 27.1 & 27.1 & -1206.3953 & -1204.4638 \\
\hline SB_002 & SB_G' & Min & 23.9 & 26.2 & 27.1 & 22.0 & -1206.3953 & -1204.4638 \\
\hline SB_022 & & Min & 24.8 & 26.5 & 28.2 & 28.2 & -1206.3936 & -1204.4623 \\
\hline SB_025 & & Min & 21.4 & 27.4 & 28.3 & 28.3 & -1206.3961 & -1204.4632 \\
\hline SB_011 & SB_H' & Min & 26.1 & 27.6 & 29.7 & 29.7 & -1206.3934 & -1204.4622 \\
\hline CS_C039 & CS_A & Min & 33.7 & 28.1 & 29.5 & 25.3 & -1206.3918 & -1204.4632 \\
\hline SB_033 & SB_I' & Min & 29.9 & 28.8 & 29.5 & 29.5 & -1206.3927 & -1204.4625 \\
\hline SB_028 & & Min & 23.0 & 29.5 & 31.0 & 31.0 & -1206.3954 & -1204.4622 \\
\hline SB_023 & & Min & 30.8 & 30.6 & 31.7 & 31.7 & -1206.3924 & -1204.4618 \\
\hline CS C009 & CS_L & Min & 39.1 & 31.1 & 31.8 & 28.6 & -1206.3899 & -1204.4623 \\
\hline SB 004 & & Min & 26.1 & 31.9 & 33.2 & 33.2 & -1206.3942 & -1204.4613 \\
\hline CS C001 & & Min & 34.7 & 32.4 & 33.5 & 28.9 & -1206.3912 & -1204.4614 \\
\hline SB 015 & & Min & 29.8 & 33.1 & 35.6 & 35.6 & -1206.3918 & -1204.4599 \\
\hline SB_031 & SB_I & Min & 32.3 & 33.1 & 34.4 & 34.4 & -1206.3919 & -1204.4609 \\
\hline CS_C007 & & Min & 44.0 & 33.6 & 34.6 & 28.5 & -1206.3877 & -1204.4610 \\
\hline CS_C006 & & Min & 34.4 & 34.4 & 35.5 & 30.8 & -1206.3914 & -1204.4608 \\
\hline CS_C017 & & Min & 37.7 & 34.4 & 36.4 & 28.5 & -1206.3896 & -1204.4602 \\
\hline CS_C033 & & Min & 51.1 & 34.4 & 34.7 & 31.9 & -1206.3854 & -1204.4611 \\
\hline CS_C008 & & Min & 40.6 & 34.7 & 35.7 & 29.9 & -1206.3892 & -1204.4608 \\
\hline CS_C014 & & Min & 34.1 & 34.7 & 35.6 & 30.8 & -1206.3917 & -1204.4608 \\
\hline CS_Chs2 & & Min & 38.3 & 36.0 & 36.2 & 33.9 & -1206.3906 & -1204.4608 \\
\hline CS_C013 & & Min & 40.5 & 36.0 & 37.1 & 31.4 & -1206.3891 & -1204.4602 \\
\hline CS_C012 & CS_B & Min & 33.4 & 36.2 & 37.9 & 30.2 & -1206.3915 & -1204.4598 \\
\hline CS_C016 & & Min & 40.0 & 36.6 & 38.3 & 32.2 & -1206.3888 & -1204.4594 \\
\hline CS_C027 & & Min & 40.0 & 36.6 & 38.4 & 32.2 & -1206.3888 & -1204.4594 \\
\hline CS_Chs1 & & Min & 42.9 & 36.9 & 38.3 & 33.7 & -1206.3882 & -1204.4598 \\
\hline SB_019 & & Min & 34.7 & 37.1 & 39.8 & 39.8 & -1206.3900 & -1204.4584 \\
\hline CS_C021 & & Min & 37.4 & 37.4 & 37.9 & 35.2 & -1206.3908 & -1204.4602 \\
\hline SB_016 & & Min & 33.8 & 37.8 & 40.3 & 40.3 & -1206.3903 & -1204.4582 \\
\hline SB_027 & & Min & 34.3 & 37.9 & 39.0 & 39.0 & -1206.3911 & -1204.4591 \\
\hline SB 018 & & Min & 33.9 & 38.9 & 40.3 & 40.3 & -1206.3906 & -1204.4580 \\
\hline CS_C031 & & Min & 48.9 & 39.0 & 39.4 & 37.2 & -1206.3863 & -1204.4594 \\
\hline CS_003 & & Min & 56.0 & 39.0 & 39.8 & 37.2 & -1206.3836 & -1204.4594 \\
\hline CS_CS1 & & Min & 46.3 & 39.9 & 40.6 & 35.6 & -1206.3872 & -1204.4590 \\
\hline CS_C023 & & Min & 46.3 & 39.9 & 40.6 & 35.5 & -1206.3872 & -1204.4589 \\
\hline CS_C010 & & Min & 45.8 & 40.2 & 40.6 & 38.9 & -1206.3876 & -1204.4591 \\
\hline CS C044 & & Min & 36.8 & 40.5 & 41.5 & 36.7 & -1206.3906 & -1204.4586 \\
\hline CS_C025 & & Min & 34.8 & 40.8 & 42.4 & 35.6 & -1206.3910 & -1204.4580 \\
\hline CS_C003 & & Min & 40.2 & 41.0 & 43.1 & 31.5 & -1206.3886 & -1204.4576 \\
\hline CS_C040 & & Min & 42.8 & 41.1 & 41.9 & 36.3 & -1206.3885 & -1204.4585 \\
\hline CS_C004 & & Min & 36.6 & 41.1 & 42.4 & 36.2 & -1206.3905 & -1204.4581 \\
\hline CS_C018 & & Min & 37.3 & 42.0 & 44.1 & 34.8 & -1206.3898 & -1204.4573 \\
\hline CS C026 & & Min & 50.2 & 43.7 & 45.1 & 39.5 & -1206.3852 & -1204.4570 \\
\hline CS_001 & & Min & 55.6 & 44.4 & 45.5 & 41.7 & -1206.3837 & -1204.4573 \\
\hline CS_C043 & & Min & 42.3 & 45.7 & 47.3 & 39.5 & -1206.3881 & -1204.4562 \\
\hline CS C035 & & Min & 50.6 & 45.9 & 46.5 & 42.4 & -1206.3856 & -1204.4567 \\
\hline
\end{tabular}

Geometry Optimization
Thermal Corrections

MP2 Single-point
B3LYP/6-31+g(d,p)

B3LYP/6-31+g(d,p)

MP2/6-311++g(2d,2p)//B3LYP/6-31+g(d,p) 
Scheme 5a. Geometry optimized conformers of $[\mathrm{Arg}+\mathrm{K}]^{+}(\mathrm{B} 3 \mathrm{LYP} / 6-31+\mathrm{g}(\mathrm{d}, \mathrm{p}))$.

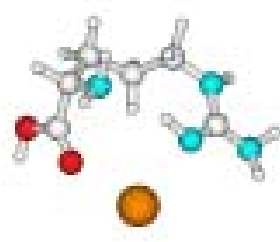

CS_001

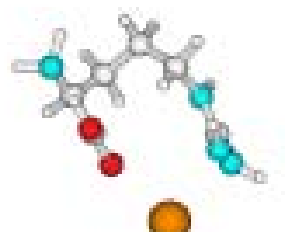

CS_C007

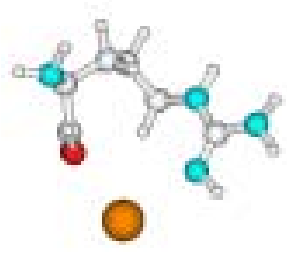

CS_C016

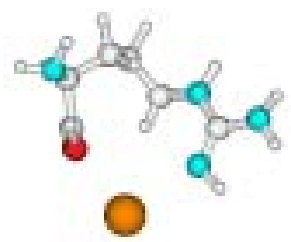

CS_C027

a. og. 0

CS_C044

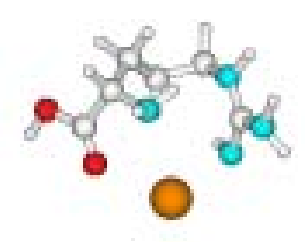

CS_003

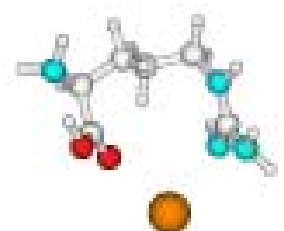

CS_C008

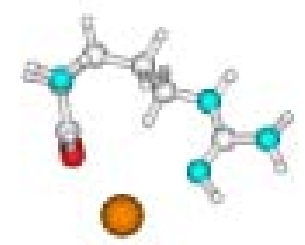

CS_C017

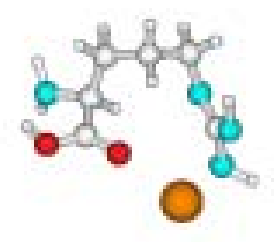

CS C031

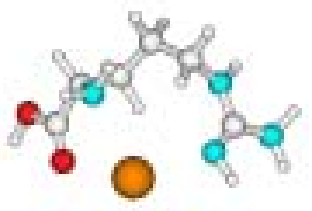

CS_Chs1

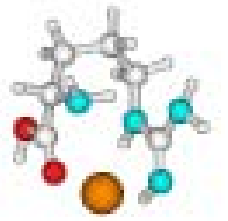

CS_016

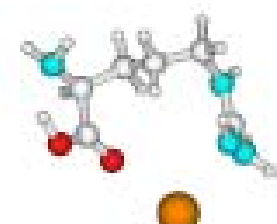

CS_C009

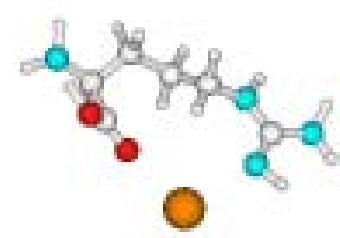

$$
\text { CS_C018 }
$$

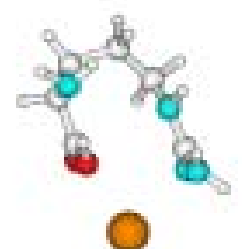

CS_C033

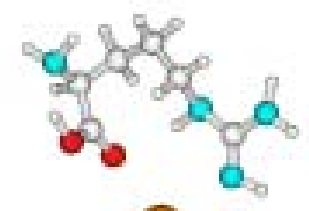

cs Chs2

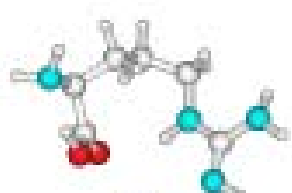

0

$$
\text { CS_C001 }
$$

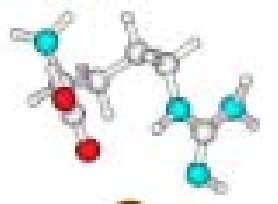

O

CS_C010

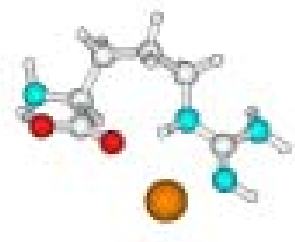

CS_C021

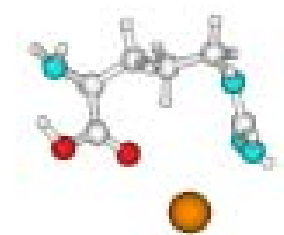

CS_C035

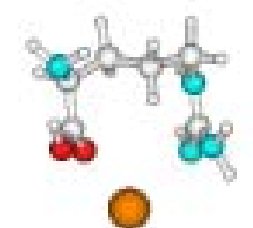

${ }^{C S}-44^{1}$

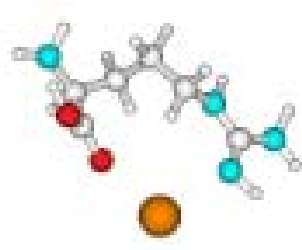

$$
\text { CS_C003 }
$$

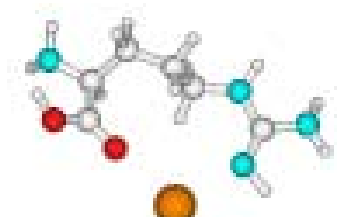

CS_C012

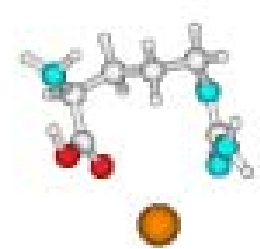

$$
\text { CS_C023 }
$$

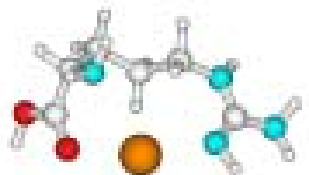

CS C039

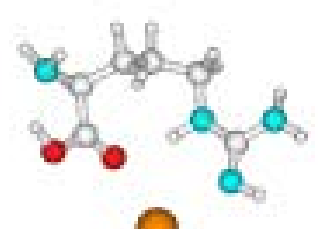

○

$$
\text { CS_CO04 }
$$

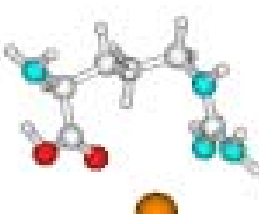

0

$$
\text { CS_C013 }
$$

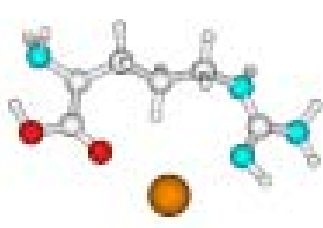

CS_C025

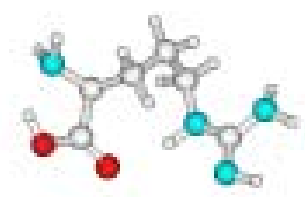

0

$$
\text { CS_C040 }
$$

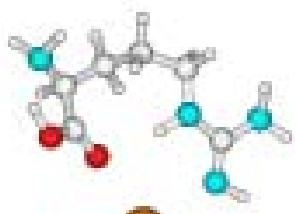

0

CS_C006

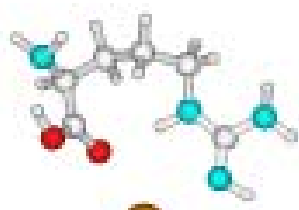

O

$$
\text { CS_C014 }
$$

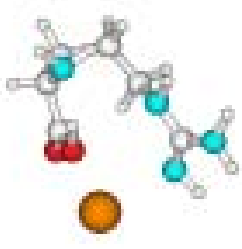

$$
\text { CS_C026 }
$$

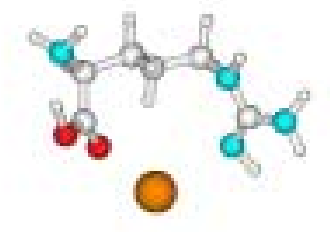

CS_C043 
Scheme $5 b$. Geometry optimized conformers of $[A r g+K]^{+}(B 3 L Y P / 6-31+g(d, p))$.

-0.0 a

SB_001

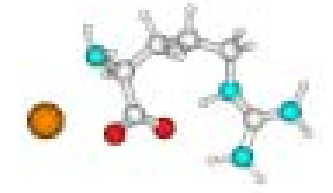

SB_002

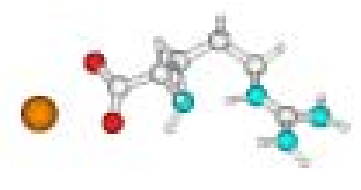

SB_009

SB_018

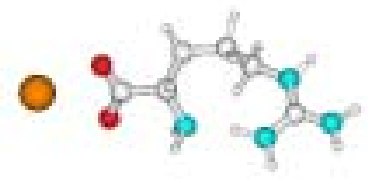

SB_028

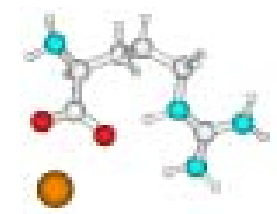

SB_011

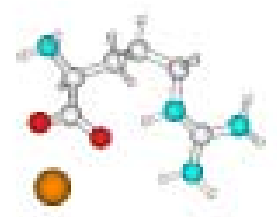

SB_019

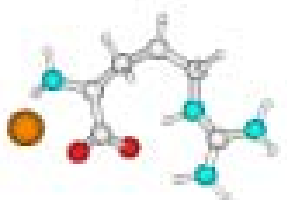

SB_004

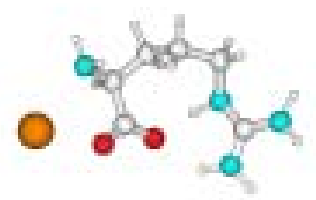

SB_012

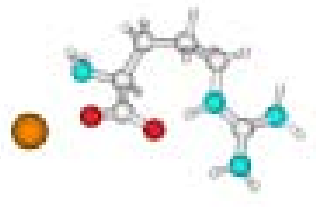

SB_022

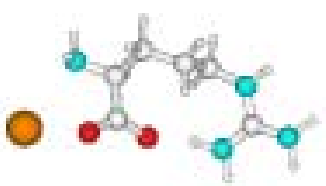

SB_031

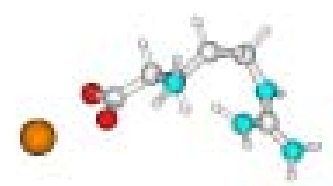

SB_032

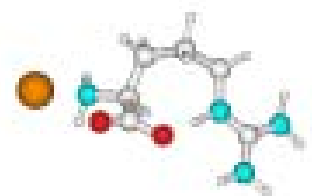

SB_005

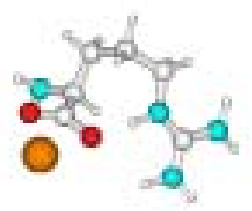

SB_013

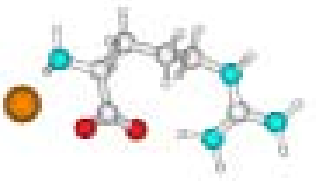

SB_023

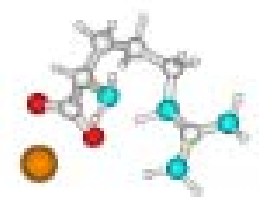

SB_006

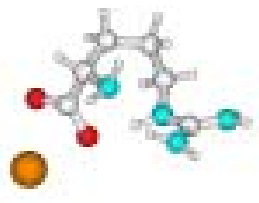

SB_014

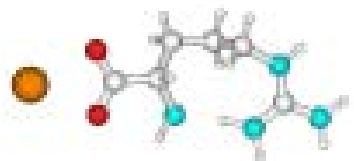

SB_025

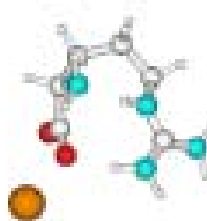

SB_007

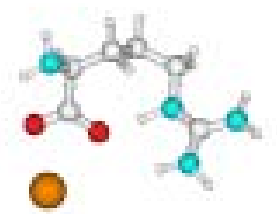

SB_015

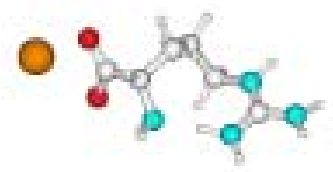

SB_026 
Figure 5. Vibrational spectra for $[A r g+K]^{+}$conformers from B3LYP/6-31+g(d,p) calculations. Spectra were constructed using a Gaussian convolution (FWHM=20 $\mathrm{cm}-1)$ and a scaling factor of 0.98 .
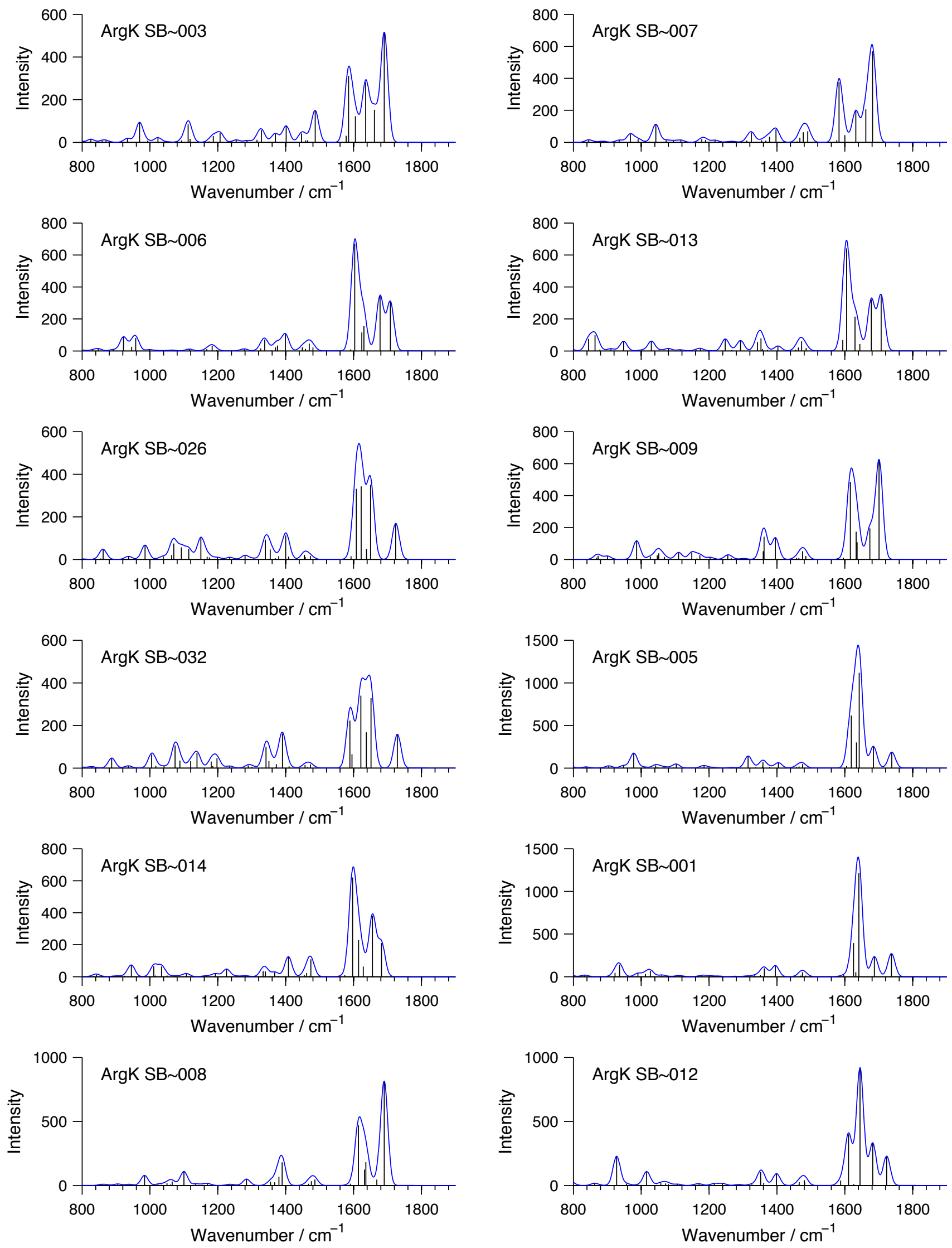


\section{Figure 5 continued.}
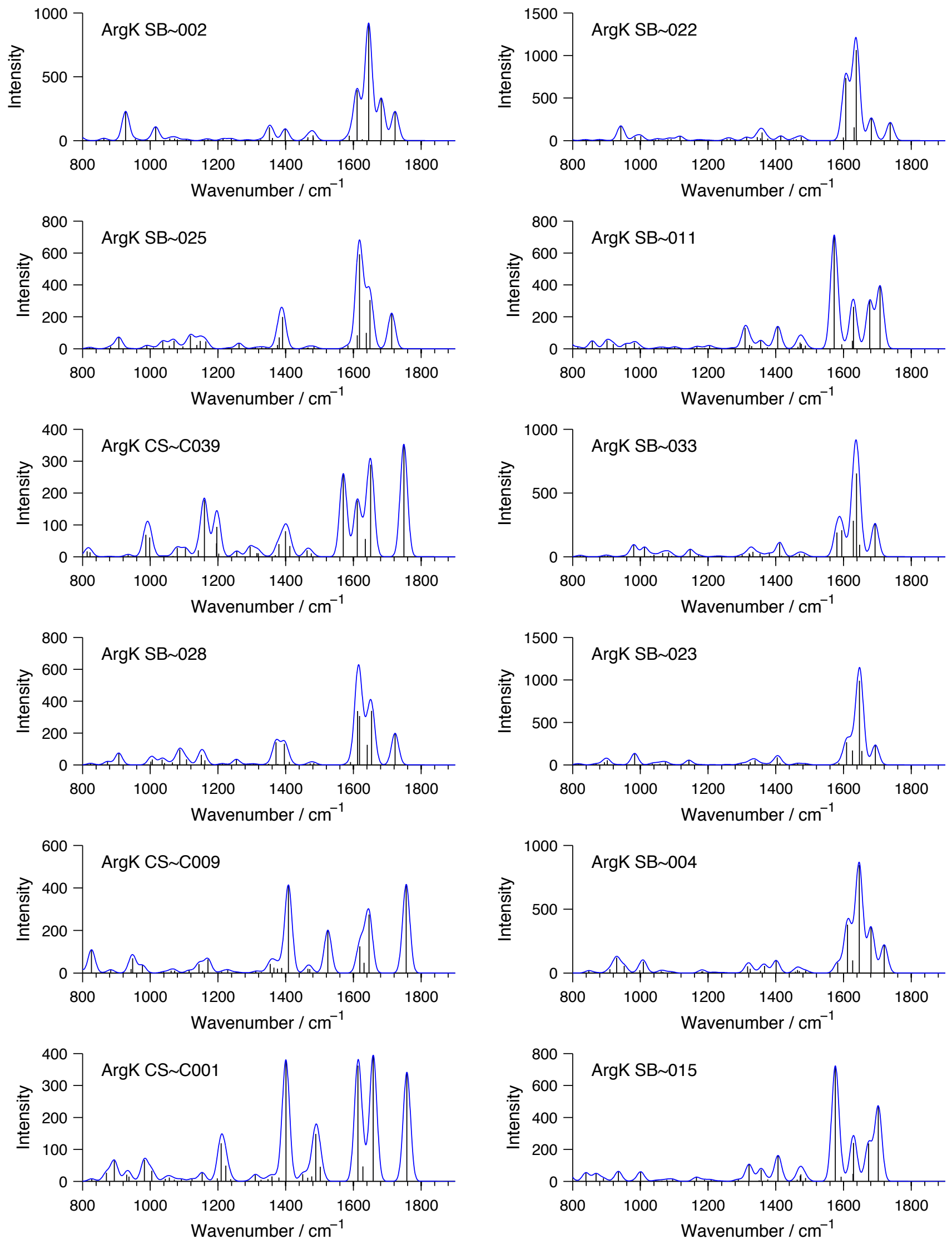
Figure 5 continued.
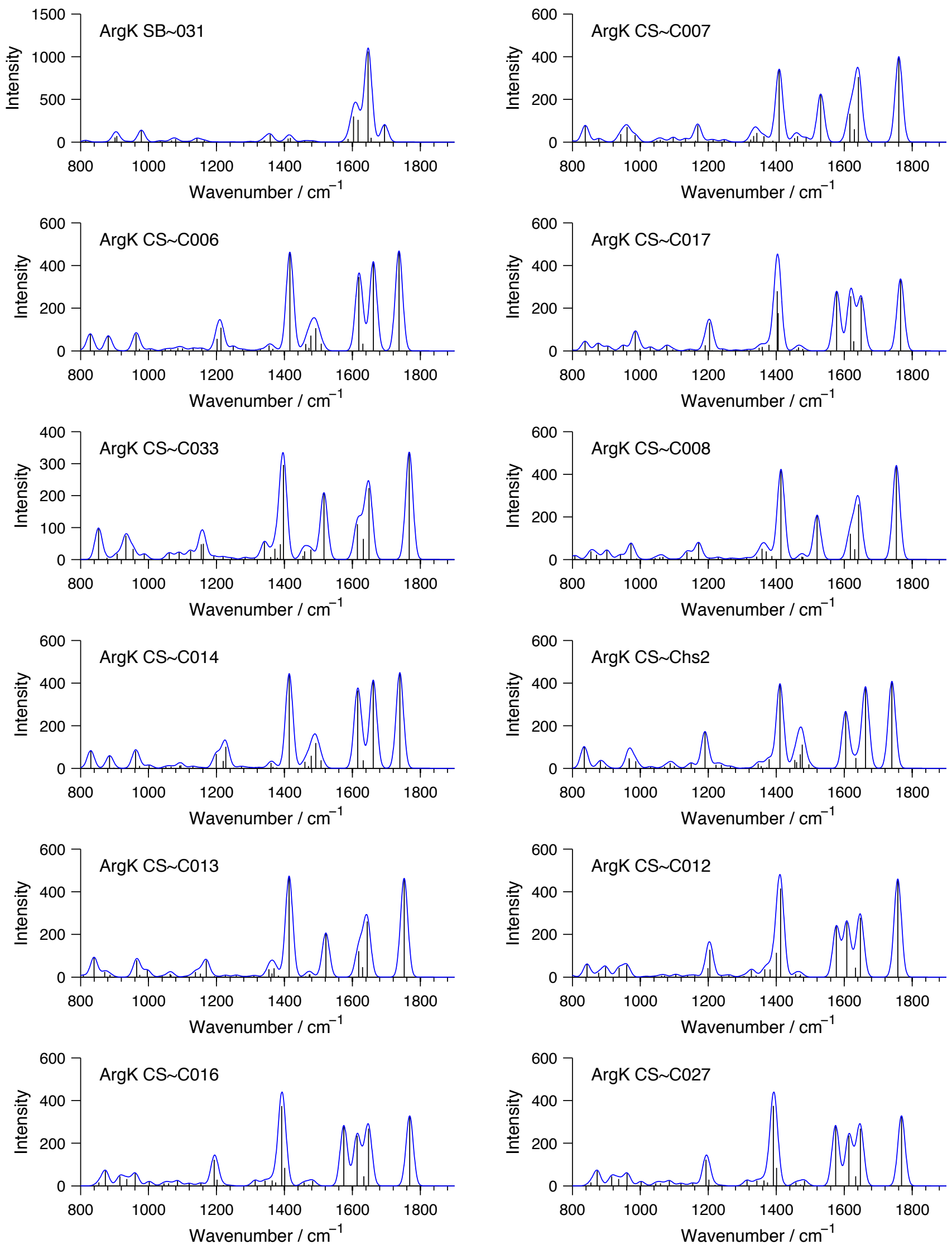
Figure 5 continued.
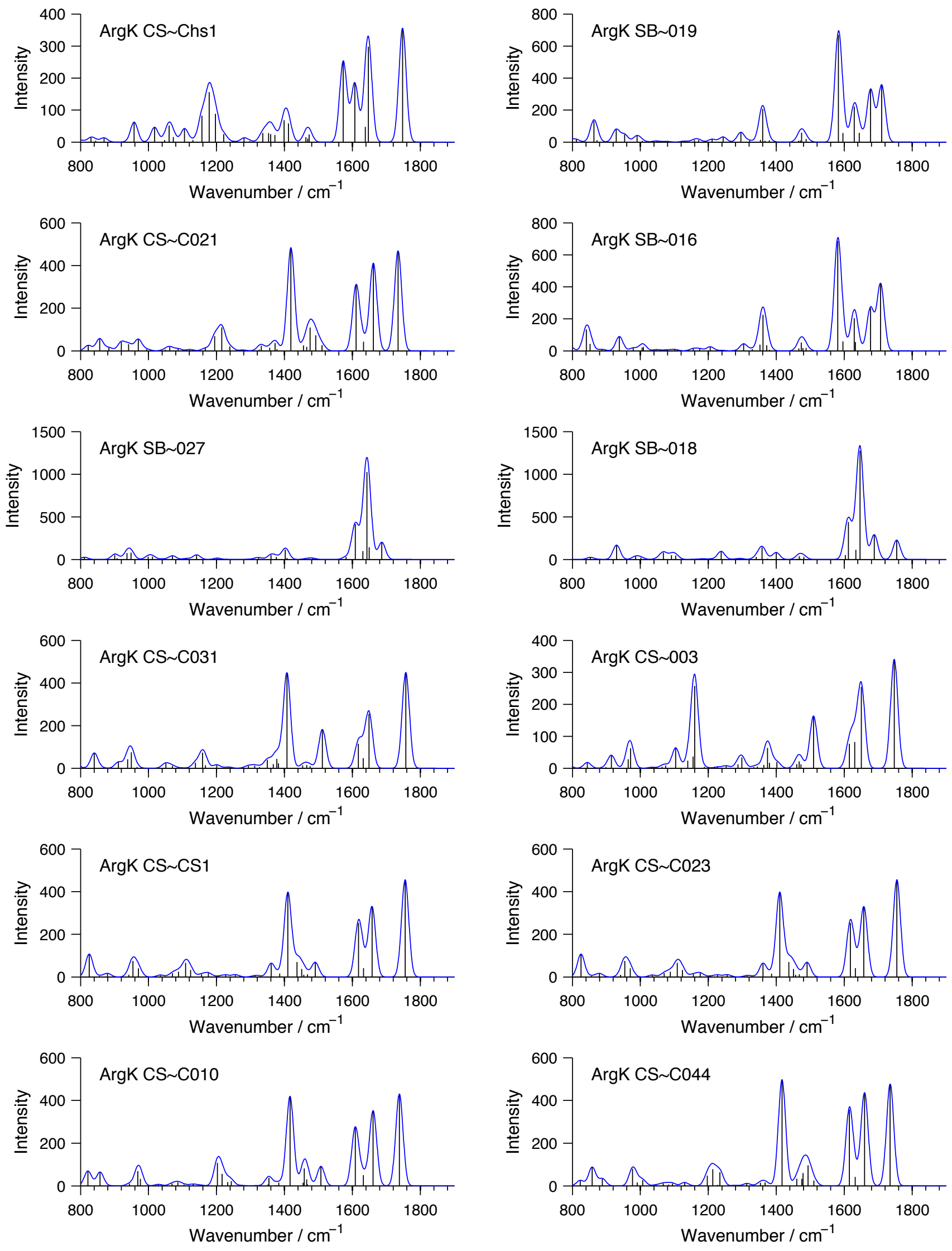
Figure 5 continued.
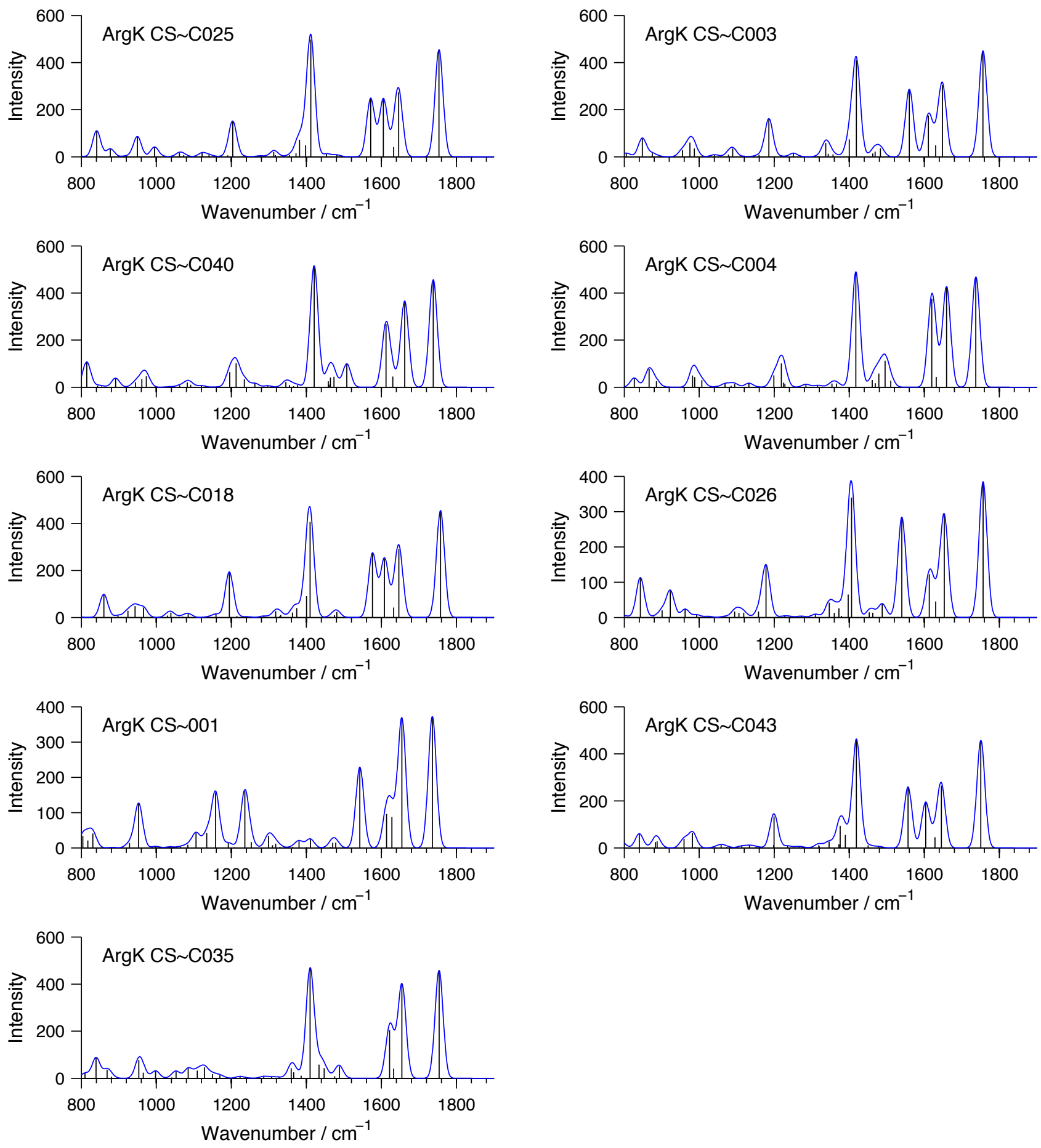
Table 7. Summary of electronic and thermal energies calculated for [Arg $+\mathrm{Rb}]^{+}$.

\begin{tabular}{|c|c|c|c|c|c|c|c|c|}
\hline & & & B3LYP & & MP2 & & & \\
\hline ArgRb & Name & Converge & $\begin{array}{c}\Delta E_{o} / \\
k J \text { mol-1 }\end{array}$ & $\begin{array}{c}\Delta E_{o} / \\
k J \quad \text { mol-1 }\end{array}$ & $\begin{array}{c}\Delta H(298) / \\
\text { kJ mol-1 }\end{array}$ & $\begin{array}{c}\Delta G(298) / \\
\text { kJ mol-1 }\end{array}$ & $\begin{array}{c}\text { B3LYP E } \\
/ \mathbf{h}\end{array}$ & $\begin{array}{c}\text { MP2 E } \\
/ / \mathbf{h}\end{array}$ \\
\hline SB_003 & SB_D & Min & 0.0 & 0.0 & 0.0 & 0.0 & -630.5970 & -628.9616 \\
\hline SB_007 & & Min & 9.3 & 7.2 & 7.3 & 7.1 & -630.5934 & -628.9589 \\
\hline SB_006 & SB_J & Min & 19.1 & 14.7 & 16.9 & 10.1 & -630.5887 & -628.9551 \\
\hline SB_013 & SB_K & Min & 15.9 & 15.8 & 17.9 & 11.9 & -630.5896 & -628.9543 \\
\hline SB_005 & & Min & 20.7 & 20.9 & 22.7 & 17.1 & -630.5871 & -628.9517 \\
\hline SB_026 & SB_F' & Min & 16.8 & 21.2 & 21.7 & 18.4 & -630.5899 & -628.9529 \\
\hline SB_009 & SB_E & Min & 13.9 & 23.3 & 25.0 & 17.5 & -630.5904 & -628.9515 \\
\hline SB_032 & SB_F & Min & 15.0 & 23.6 & 24.0 & 21.2 & -630.5904 & -628.9518 \\
\hline SB_014 & & Min & 25.6 & 24.1 & 25.1 & 22.0 & -630.5870 & -628.9522 \\
\hline SB_022 & & Min & 23.8 & 24.5 & 26.1 & 20.5 & -630.5863 & -628.9506 \\
\hline SB_001 & SB_G & Min & 20.8 & 25.4 & 27.0 & 19.6 & -630.5882 & -628.9511 \\
\hline SB_002 & SB_G' & Min & 24.9 & 26.0 & 26.8 & 22.4 & -630.5873 & -628.9516 \\
\hline SB_011 & SB_H' & Min & 25.5 & 27.3 & 29.3 & 21.8 & -630.5860 & -628.9499 \\
\hline SB_008 & & Min & 22.8 & 29.0 & 30.7 & 24.5 & -630.5874 & -628.9497 \\
\hline CS_C009 & CS_L & Min & 38.0 & 29.8 & 30.9 & 26.4 & -630.5825 & -628.9502 \\
\hline SB_033 & & Min & 30.7 & 30.2 & 31.1 & 28.6 & -630.5844 & -628.9492 \\
\hline SB_004 & & Min & 24.4 & 30.3 & 32.4 & 22.0 & -630.5863 & -628.9487 \\
\hline CS_C033 & & Min & 49.2 & 31.2 & 31.8 & 28.4 & -630.5783 & -628.9498 \\
\hline CS_C001 & & Min & 34.1 & 31.4 & 32.7 & 27.5 & -630.5837 & -628.9494 \\
\hline SB_031 & & Min & 32.0 & 31.6 & 32.9 & 27.4 & -630.5841 & -628.9489 \\
\hline SB_025 & & Min & 24.1 & 31.6 & 32.4 & 28.9 & -630.5873 & -628.9491 \\
\hline CS_C006 & & Min & 33.9 & 31.9 & 33.0 & 28.7 & -630.5840 & -628.9494 \\
\hline CS_C014 & & Min & 33.3 & 32.0 & 33.2 & 28.0 & -630.5842 & -628.9493 \\
\hline CS_C008 & & Min & 38.6 & 32.5 & 33.9 & 26.8 & -630.5820 & -628.9490 \\
\hline SB_015 & & Min & 29.3 & 32.7 & 35.0 & 26.2 & -630.5843 & -628.9477 \\
\hline CS_C007 & & Min & 43.2 & 32.8 & 34.0 & 28.0 & -630.5803 & -628.9489 \\
\hline CS_Chs2 & & Min & 37.4 & 33.1 & 33.3 & 31.4 & -630.5833 & -628.9495 \\
\hline SB_023 & & Min & 32.5 & 33.1 & 34.1 & 30.3 & -630.5840 & -628.9485 \\
\hline CS_C021 & & Min & 35.8 & 33.3 & 34.0 & 30.9 & -630.5836 & -628.9492 \\
\hline SB_028 & & Min & 25.8 & 33.7 & 35.2 & 28.9 & -630.5865 & -628.9482 \\
\hline CS_C039 & CS_A & Min & 38.1 & 33.9 & 35.7 & 30.1 & -630.5821 & -628.9484 \\
\hline CS_C017 & & Min & 37.9 & 34.0 & 36.0 & 29.1 & -630.5819 & -628.9480 \\
\hline CS_C013 & & Min & 38.6 & 34.9 & 36.4 & 29.2 & -630.5820 & -628.9481 \\
\hline CS_C027 & & Min & 39.3 & 35.2 & 37.3 & 29.4 & -630.5811 & -628.9474 \\
\hline CS_C016 & & Min & 39.3 & 35.2 & 37.4 & 29.5 & -630.5811 & -628.9474 \\
\hline CS_C012 & CS_B & Min & 34.5 & 35.9 & 37.8 & 29.3 & -630.5833 & -628.9474 \\
\hline CS_C010 & & Min & 44.1 & 35.9 & 36.5 & 33.8 & -630.5804 & -628.9482 \\
\hline SB_019 & & Min & 34.2 & 37.1 & 39.6 & 31.1 & -630.5825 & -628.9461 \\
\hline CS_C023 & & Min & 44.0 & 37.1 & 38.1 & 32.5 & -630.5802 & -628.9475 \\
\hline CS_CS1 & & Min & 44.0 & 37.2 & 38.1 & 32.5 & -630.5802 & -628.9475 \\
\hline SB_018 & & Min & 33.7 & 37.4 & 38.8 & 32.5 & -630.5828 & -628.9460 \\
\hline CS_C044 & & Min & 35.7 & 37.5 & 38.6 & 33.9 & -630.5834 & -628.9474 \\
\hline CS_C031 & & Min & 47.9 & 37.7 & 38.5 & 34.5 & -630.5788 & -628.9473 \\
\hline CS_C004 & & Min & 35.3 & 37.9 & 39.3 & 32.6 & -630.5833 & -628.9470 \\
\hline SB_016 & & Min & 33.3 & 37.9 & 40.2 & 31.7 & -630.5829 & -628.9458 \\
\hline CS_C040 & & Min & 41.9 & 38.9 & 39.5 & 35.4 & -630.5814 & -628.9472 \\
\hline SB_027 & & Min & 35.4 & 39.2 & 40.2 & 36.5 & -630.5829 & -628.9461 \\
\hline CS_C025 & & Min & 35.0 & 39.5 & 41.3 & 34.5 & -630.5832 & -628.9461 \\
\hline CS_C003 & & Min & 41.1 & 40.2 & 42.2 & 33.5 & -630.5807 & -628.9457 \\
\hline CS_003 & & Min & 57.0 & 40.6 & 41.8 & 38.5 & -630.5753 & -628.9462 \\
\hline CS_C018 & & Min & 38.2 & 41.0 & 43.5 & 32.9 & -630.5816 & -628.9451 \\
\hline CS_C043 & & Min & 41.3 & 41.7 & 43.4 & 36.6 & -630.5808 & -628.9453 \\
\hline CS_Chs1 & & Min & 46.4 & 41.7 & 43.5 & 38.0 & -630.5790 & -628.9454 \\
\hline CS_C026 & & Min & 50.1 & 41.9 & 43.5 & 37.2 & -630.5774 & -628.9452 \\
\hline CS_001 & & Min & 54.7 & 42.7 & 43.8 & 40.4 & -630.5765 & -628.9457 \\
\hline CS_C035 & & Min & 48.9 & 44.1 & 45.1 & 39.6 & -630.5784 & -628.9449 \\
\hline CS_016 & & Min & 69.8 & 46.3 & 46.1 & 49.1 & -630.5712 & -628.9448 \\
\hline
\end{tabular}

\begin{tabular}{|ll|}
\hline Geometry Optimization & B3LYP/6-31+g(d,p) \\
Thermal Corrections & B3LYP/6-31+g(d,p) \\
MP2 Single-point & MP2/6-311++g(2d,2p)//B3LYP/6-31+g(d,p) \\
\hline
\end{tabular}


Scheme 6a. Geometry optimized conformers of $[A r g+R b]^{+}(B 3 L Y P / 6-31+g(d, p))$.

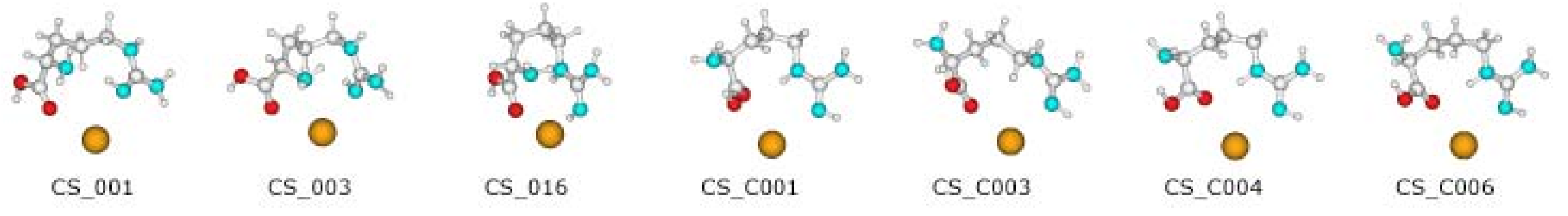

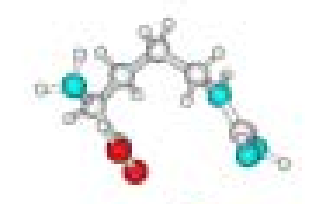

O

CS_C007

$8 b_{0}^{28} 0=0$

O

CS_C016

$8 e_{0}^{20}=0$

○

CS_C027

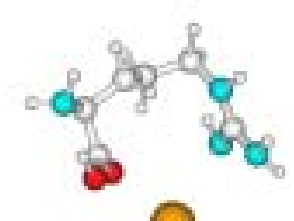

0

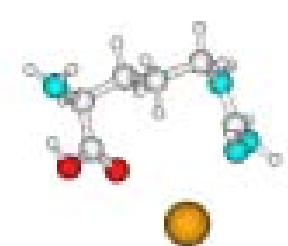

CS_C009

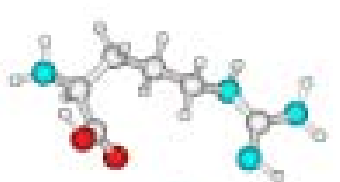

0

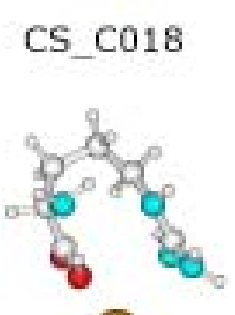

○

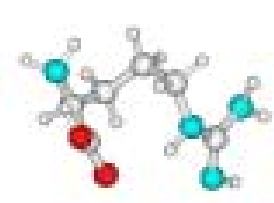

0

CS_C010

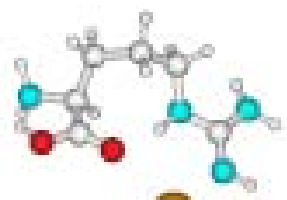

0

$$
\text { CS_C021 }
$$

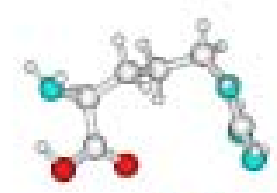

0

CS_C035

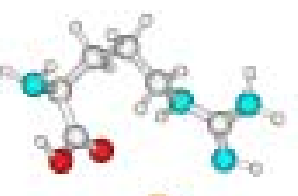

0

CS_Chs2

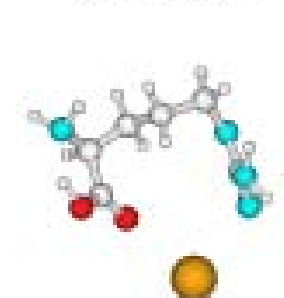

CS_CS1

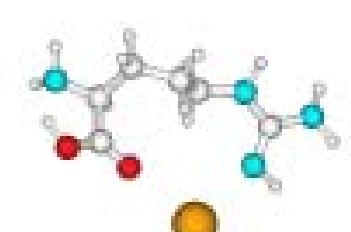

0

CS_C012

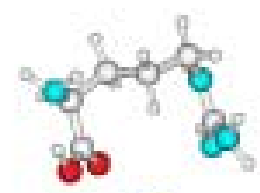

O

$$
\text { CS_C023 }
$$

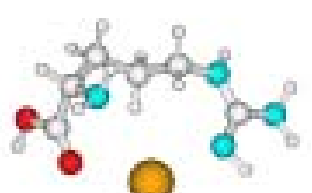

CS_C039

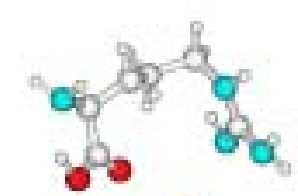

0

CS_C013

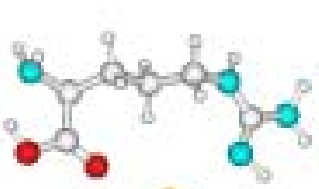

0

CS_C025

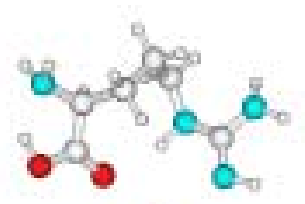

0

CS_C040

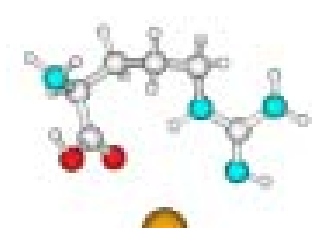

CS_C014

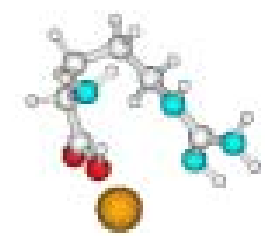

CS_C026

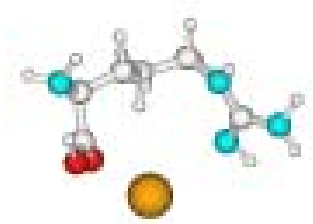

CS_C043
-0. 88.0

0

CS_C044
CS_Chs1

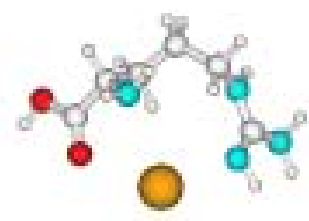$$
\text { Cs_ }
$$ 
Scheme $6 b$. Geometry optimized conformers of $[A r g+R b]^{+}(B 3 L Y P / 6-31+g(d, p))$.

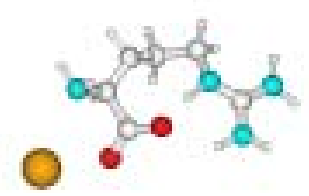

SB_001

0 - 30 ep

SB_008

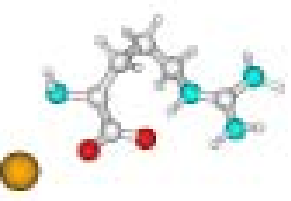

SB_018

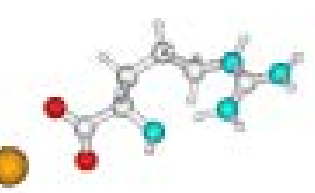

SB 028

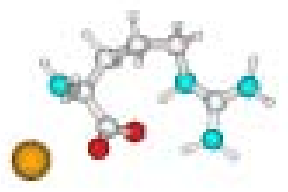

SB_002

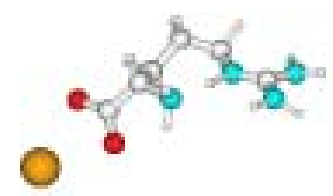

SB_009

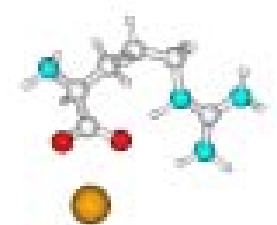

SB_019

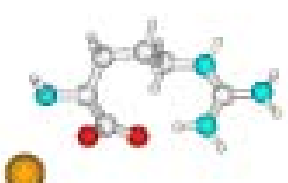

SB_031

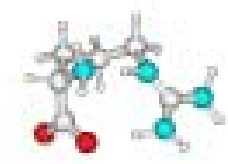

0

SB_003

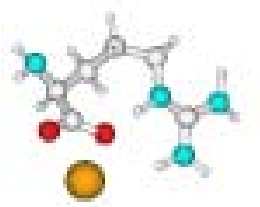

SB_011

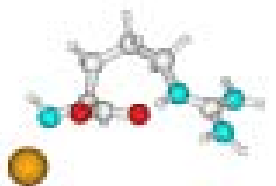

SB_022

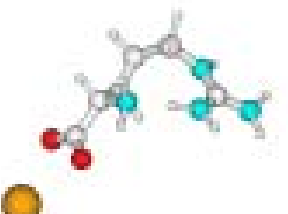

SB 032

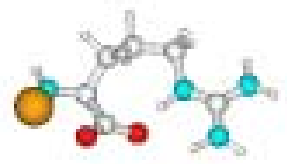

SB_004

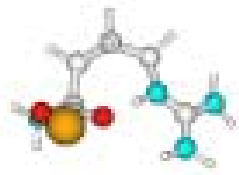

SB_013

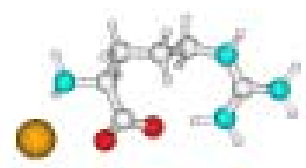

SB_023

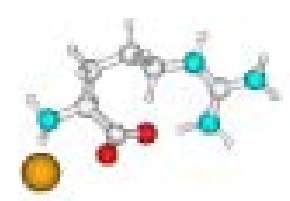

SB 033

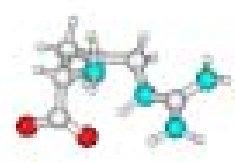

0

SB_005

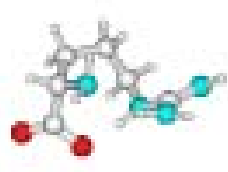

0

SB_014

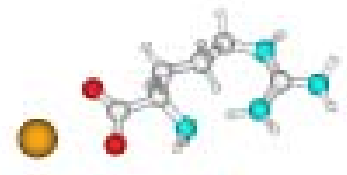

SB 025

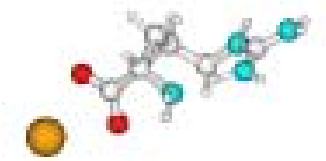

SB_026

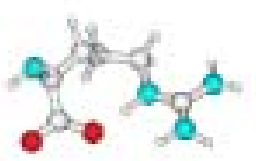

0

SB_016

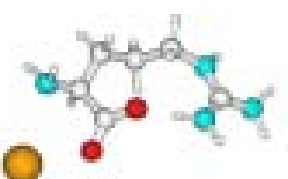

SB_027 
Figure 6. Vibrational spectra for $[A r g+R b]^{+}$conformers from $B 3 L Y P / 6-31+g(d, p)$ calculations. Spectra were constructed using a Gaussian convolution (FWHM=20 $\mathrm{cm}-1)$ and a scaling factor of 0.98 .
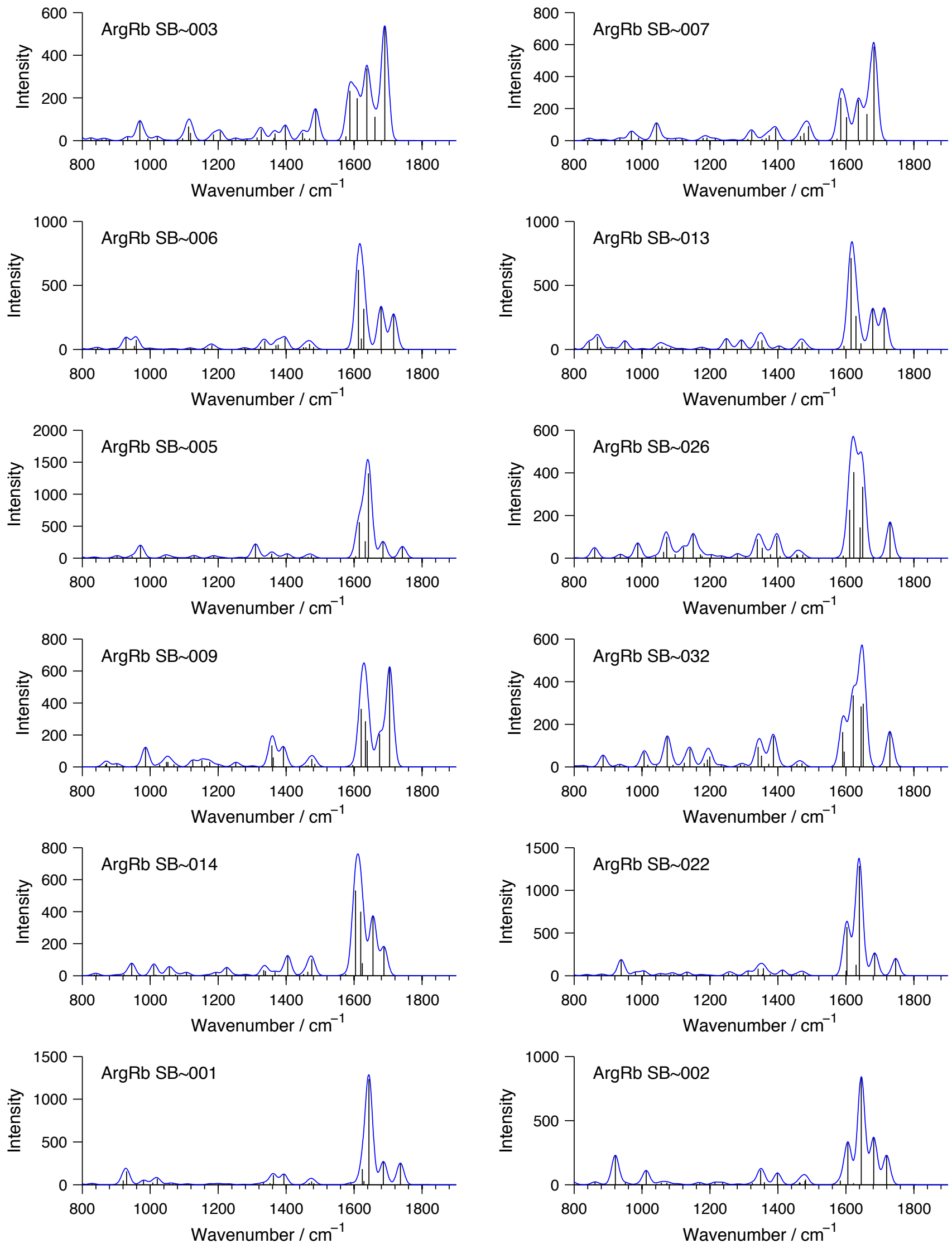
Figure 6 continued.
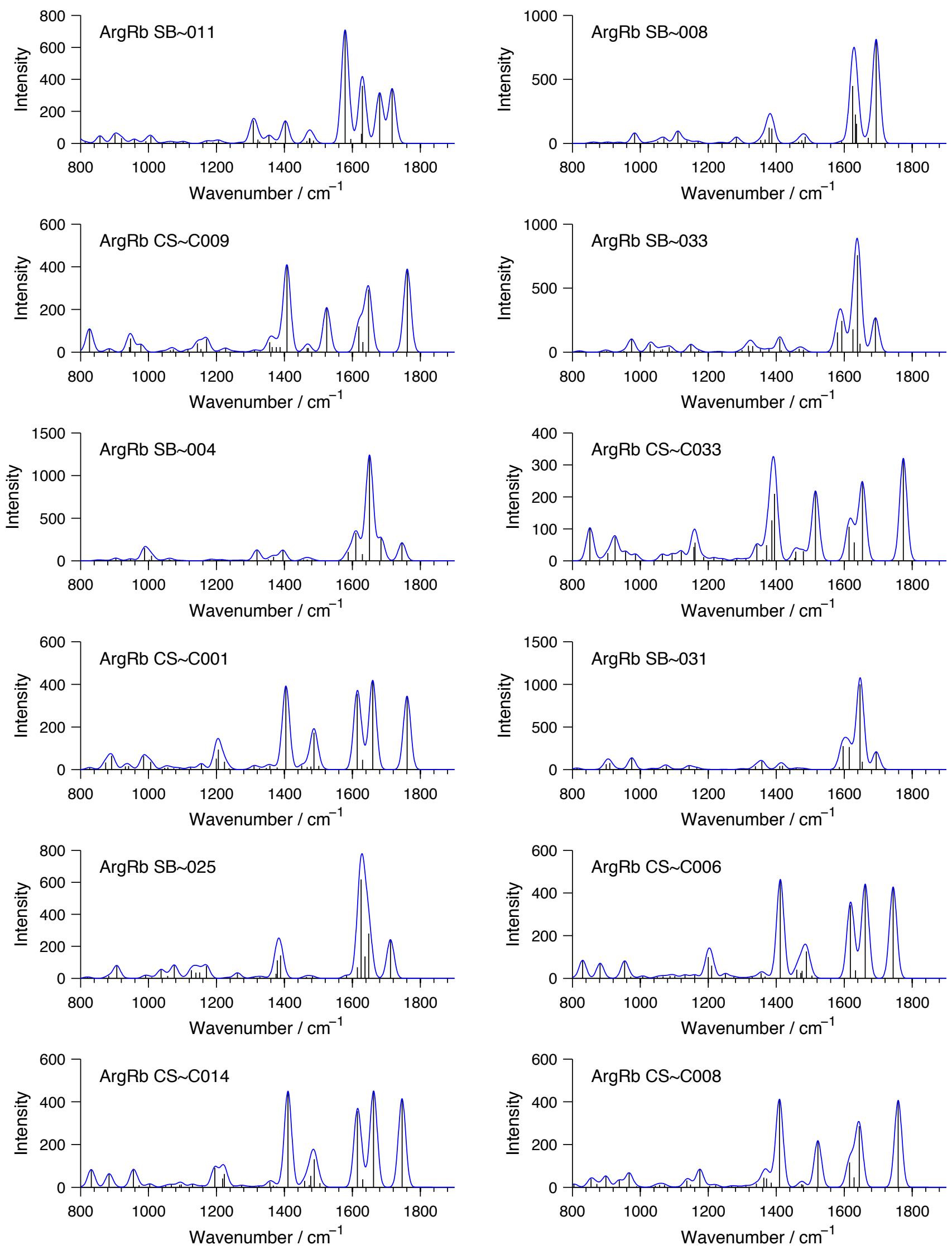
Figure 6 continued.
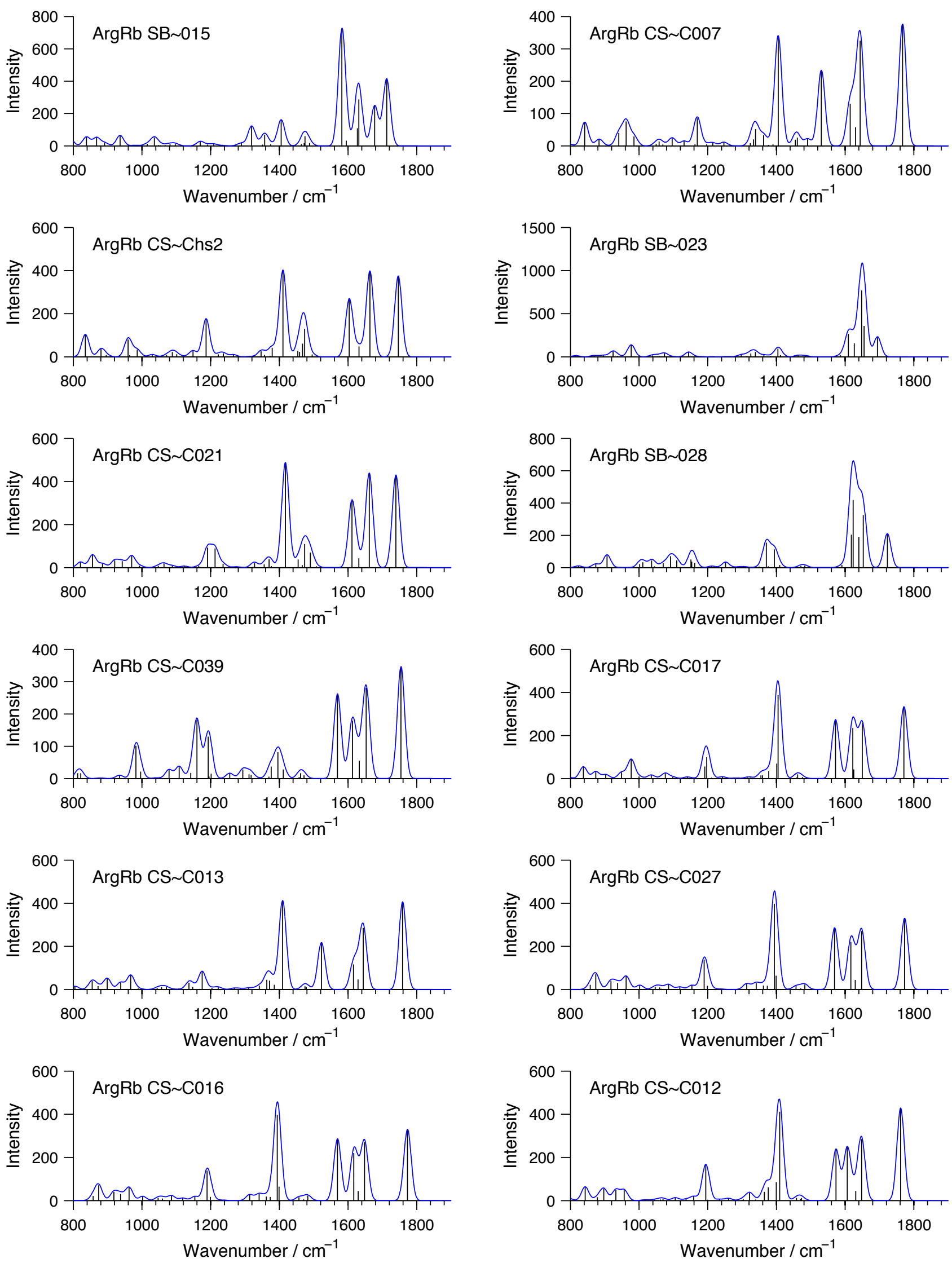
Figure 6 continued.
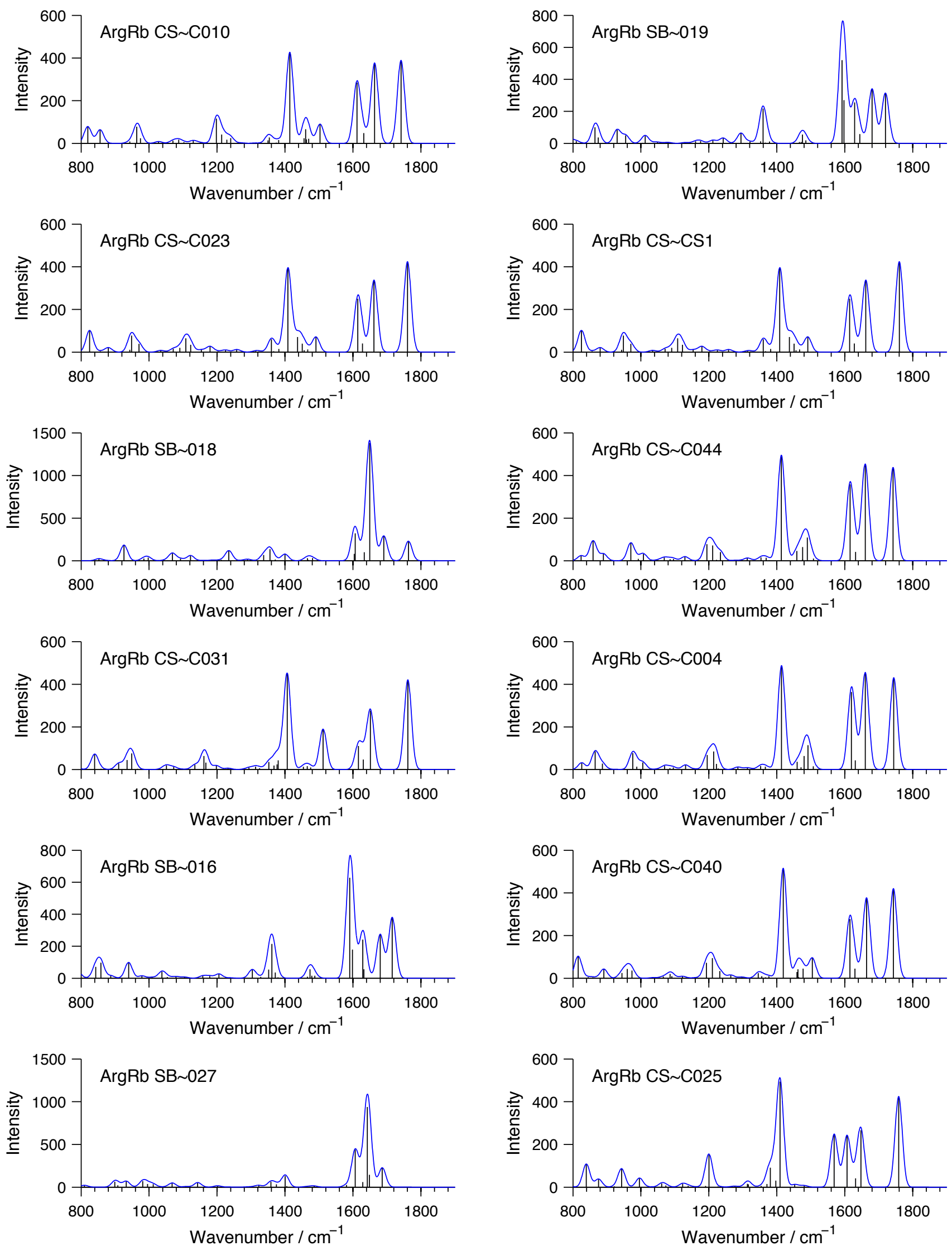
Figure 6 continued.
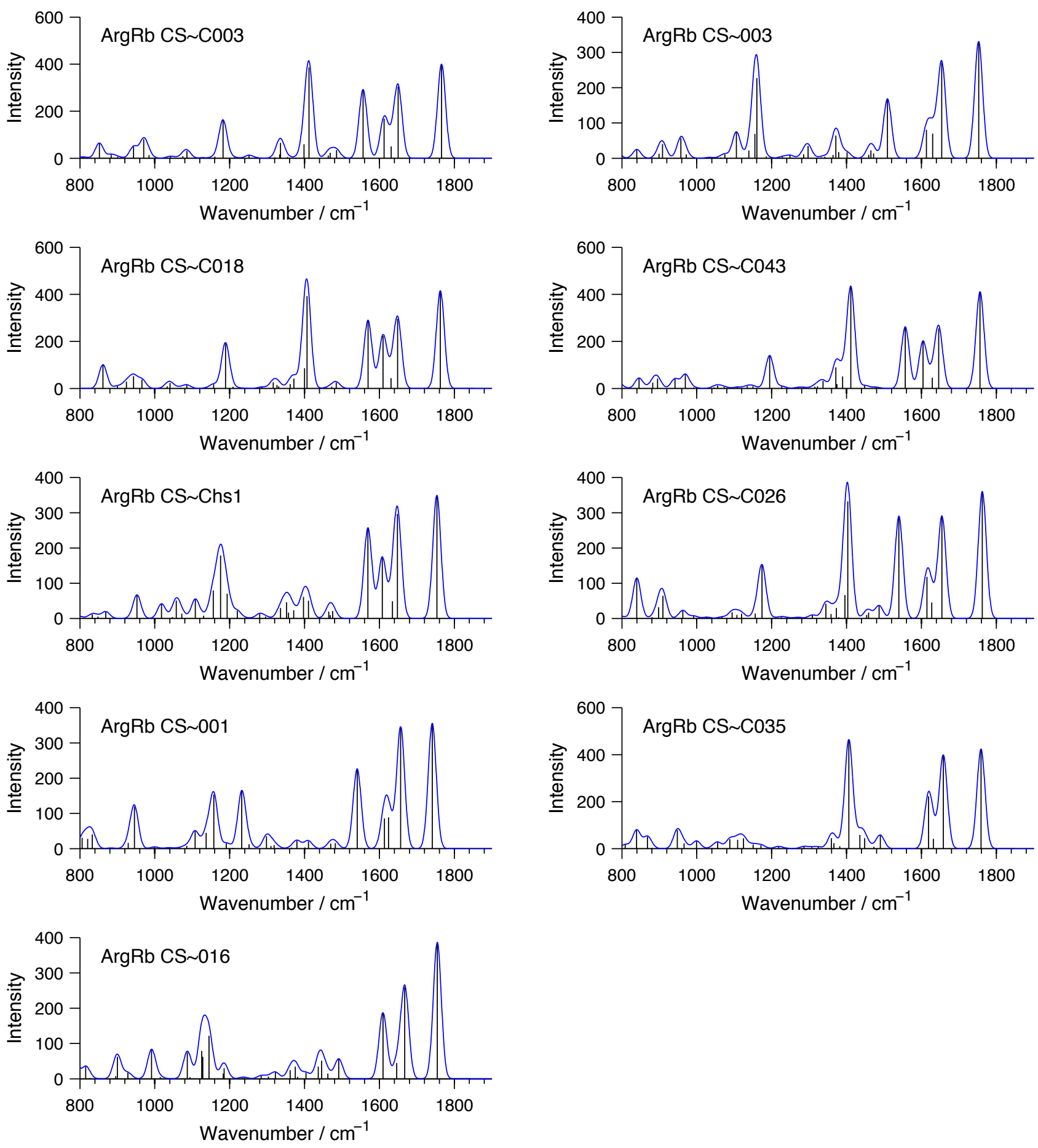
Table 8. Summary of electronic and thermal energies calculated for $[\mathrm{Arg}+\mathrm{Cs}]^{+}$.

\begin{tabular}{|c|c|c|c|c|c|c|c|c|}
\hline & & & B3LYP & & MP2 & & & \\
\hline ArgCs & Name & Converge & $\begin{array}{c}\Delta E_{o} / \\
k J \text { mol-1 }\end{array}$ & $\begin{array}{c}\Delta E_{o} / \\
k J \quad \text { mol-1 }\end{array}$ & $\begin{array}{c}\Delta H(298) / \\
\text { kJ mol-1 }\end{array}$ & $\begin{array}{c}\Delta G(298) / \\
\text { kJ mol-1 }\end{array}$ & $\begin{array}{c}\text { B3LYP E } \\
/ \mathbf{h}\end{array}$ & $\begin{array}{c}\text { MP2 E } \\
/ / \mathbf{h}\end{array}$ \\
\hline SB_003 & SB_D & Min & 0.0 & 0.0 & 0.0 & 0.0 & -626.6516 & -625.0129 \\
\hline SB_007 & & Min & 8.9 & 6.7 & 6.8 & 6.3 & -626.6482 & -625.0103 \\
\hline SB_006 & SB_J & Min & 19.6 & 14.8 & 16.9 & 10.4 & -626.6434 & -625.0065 \\
\hline SB_013 & SB_K & Min & 15.3 & 15.0 & 17.2 & 10.7 & -626.6443 & -625.0057 \\
\hline SB_005 & & Min & 20.7 & 20.0 & 21.7 & 16.2 & -626.6417 & -625.0032 \\
\hline SB_022 & & Min & 22.7 & 23.1 & 24.4 & 19.9 & -626.6414 & -625.0025 \\
\hline SB_026 & SB_F' & Min & 18.4 & 23.2 & 23.7 & 20.6 & -626.6440 & -625.0034 \\
\hline SB_014 & & Min & 25.8 & 24.5 & 25.4 & 22.7 & -626.6415 & -625.0033 \\
\hline SB_002 & SB_G' & Min & 24.8 & 25.1 & 26.0 & 21.4 & -626.6419 & -625.0031 \\
\hline SB_001 & SB_G & Min & 21.4 & 25.5 & 26.9 & 21.2 & -626.6429 & -625.0026 \\
\hline SB_032 & SB_F & Min & 16.4 & 25.8 & 26.2 & 23.5 & -626.6444 & -625.0021 \\
\hline SB_009 & SB_E & Min & 15.8 & 26.1 & 27.7 & 21.3 & -626.6444 & -625.0017 \\
\hline SB_011 & SB_H' & Min & 24.9 & 28.0 & 29.8 & 21.8 & -626.6410 & -625.0010 \\
\hline SB_004 & & Min & 23.8 & 28.9 & 31.0 & 22.6 & -626.6411 & -625.0004 \\
\hline CS_C009 & CS_L & Min & 38.5 & 29.2 & 30.5 & 25.5 & -626.6369 & -625.0016 \\
\hline CS_C033 & & Min & 49.4 & 29.3 & 29.9 & 28.0 & -626.6330 & -625.0019 \\
\hline SB_031 & SB_I & Min & 31.2 & 30.1 & 31.4 & 26.6 & -626.6390 & -625.0007 \\
\hline SB_033 & SB_I' & Min & 33.2 & 30.8 & 31.6 & 29.5 & -626.6385 & -625.0003 \\
\hline CS_C008 & & Min & 39.0 & 31.6 & 32.9 & 26.9 & -626.6366 & -625.0007 \\
\hline CS_C001 & & Min & 35.9 & 31.7 & 33.1 & 27.8 & -626.6377 & -625.0005 \\
\hline SB_008 & & Min & 24.9 & 31.8 & 33.4 & 27.8 & -626.6413 & -624.9999 \\
\hline CS_C014 & & Min & 34.3 & 32.3 & 33.7 & 27.8 & -626.6383 & -625.0004 \\
\hline SB_015 & & Min & 28.3 & 32.4 & 34.5 & 25.4 & -626.6394 & -624.9991 \\
\hline CS_C006 & & Min & 35.2 & 32.5 & 33.8 & 28.8 & -626.6382 & -625.0005 \\
\hline CS_C021 & & Min & 36.2 & 32.9 & 33.7 & 30.8 & -626.6381 & -625.0006 \\
\hline CS_C007 & & Min & 44.1 & 33.0 & 34.0 & 29.5 & -626.6349 & -625.0003 \\
\hline SB_023 & & Min & 33.5 & 33.3 & 34.4 & 30.5 & -626.6382 & -624.9995 \\
\hline CS_C013 & & TS & 39.3 & 33.8 & 32.9 & 34.1 & -626.6363 & -624.9997 \\
\hline CS_C017 & & Min & 39.5 & 33.9 & 36.1 & 27.4 & -626.6359 & -624.9993 \\
\hline CS_C016 & & Min & 40.7 & 34.2 & 36.4 & 28.9 & -626.6354 & -624.9991 \\
\hline CS_C027 & & Min & 40.7 & 34.2 & 36.5 & 28.9 & -626.6354 & -624.9991 \\
\hline SB_025 & & Min & 26.1 & 34.4 & 35.1 & 32.2 & -626.6411 & -624.9992 \\
\hline CS_C010 & & Min & 44.3 & 35.3 & 35.9 & 32.9 & -626.6351 & -624.9998 \\
\hline SB_028 & & Min & 28.1 & 36.5 & 37.9 & 32.5 & -626.6403 & -624.9983 \\
\hline CS_C039 & CS_A & Min & 44.2 & 36.7 & 38.6 & 32.3 & -626.6343 & -624.9984 \\
\hline CS_C031 & & Min & 48.4 & 36.7 & 37.7 & 33.7 & -626.6332 & -624.9989 \\
\hline CS_C023 & & Min & 44.3 & 37.0 & 37.9 & 32.9 & -626.6349 & -624.9990 \\
\hline CS_C012 & CS_B & Min & 37.1 & 37.1 & 39.1 & 30.3 & -626.6369 & -624.9982 \\
\hline SB_019 & & Min & 33.4 & 37.4 & 39.8 & 29.9 & -626.6375 & -624.9972 \\
\hline CS_C004 & & Min & 36.5 & 38.0 & 39.5 & 32.8 & -626.6375 & -624.9982 \\
\hline SB_016 & & TS & 31.8 & 38.4 & 38.4 & 36.1 & -626.6379 & -624.9966 \\
\hline SB_027 & & Min & 36.0 & 39.4 & 40.4 & 37.2 & -626.6373 & -624.9972 \\
\hline CS_C003 & & Min & 42.7 & 39.5 & 41.8 & 32.5 & -626.6346 & -624.9971 \\
\hline CS_003 & & Min & 60.1 & 40.0 & 41.4 & 36.8 & -626.6287 & -624.9976 \\
\hline CS_C025 & & Min & 36.6 & 40.1 & 42.0 & 34.5 & -626.6371 & -624.9970 \\
\hline CS_C018 & & Min & 40.5 & 41.4 & 44.0 & 33.0 & -626.6354 & -624.9963 \\
\hline CS_C026 & & Min & 52.0 & 41.7 & 43.3 & 37.3 & -626.6314 & -624.9966 \\
\hline CS_001 & & Min & 55.0 & 42.3 & 43.5 & 39.3 & -626.6309 & -624.9970 \\
\hline CS_C035 & & Min & 49.0 & 43.9 & 44.9 & 39.3 & -626.6330 & -624.9962 \\
\hline CS_016 & & Min & 71.3 & 44.7 & 44.6 & 47.4 & -626.6253 & -624.9967 \\
\hline SB_018 & & Min & 32.2 & & & & -626.6376 & \\
\hline
\end{tabular}

Geometry Optimization

Thermal Corrections

MP2 Single-point
$B 3 L Y P / 6-31+g(d, p)$

$B 3 L Y P / 6-31+g(d, p)$

MP2/6-311++g(2d,2p)//B3LYP/6-31+g(d,p) 
Scheme 7a. Geometry optimized conformers of $[\mathrm{Arg}+\mathrm{Cs}]^{+}(\mathrm{B} 3 \mathrm{LYP} / 6-31+\mathrm{g}(\mathrm{d}, \mathrm{p}))$.

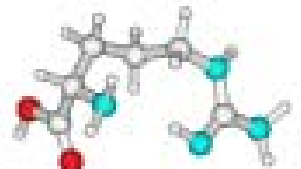

0

CS_001

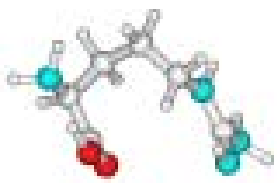

0

CS_C007

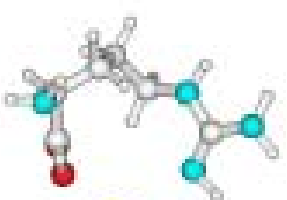

0

CS_C016

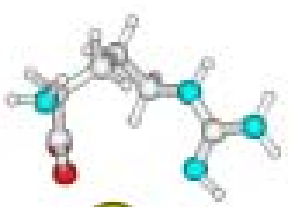

0

CS_C027

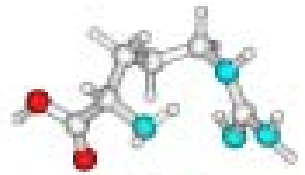

0

CS_003

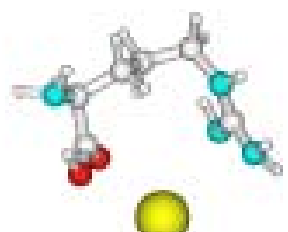

CS_COOB

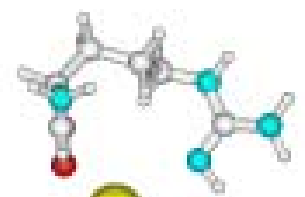

0

CS_C017

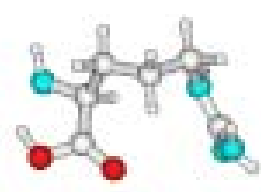

0

CS_C031

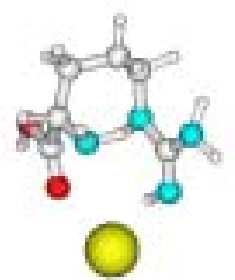

CS_016

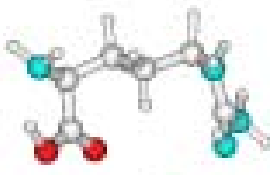

O

CS_C009

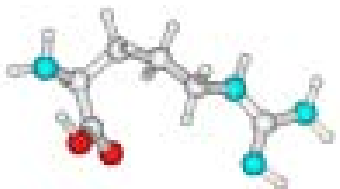

0

CS C018

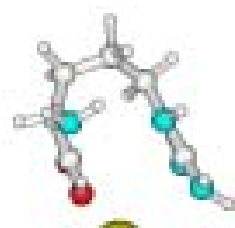

CS_C033

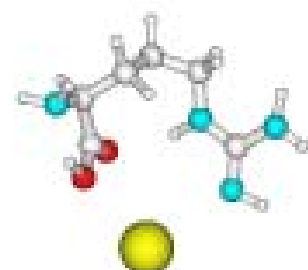

CS_C001

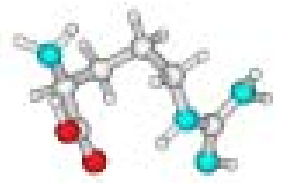

O

CS_C010

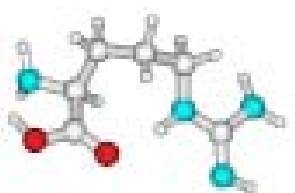

0

CS_C021

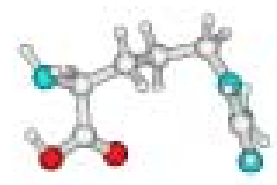

0

CS_C035

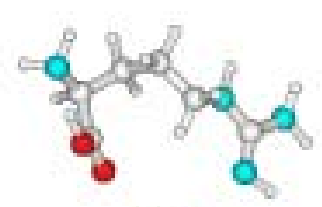

0

CS_C003

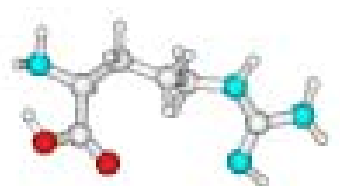

0

$$
\text { CS_C012 }
$$

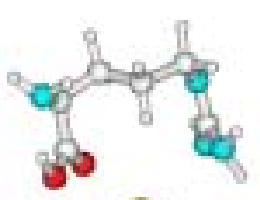

0

CS_C023

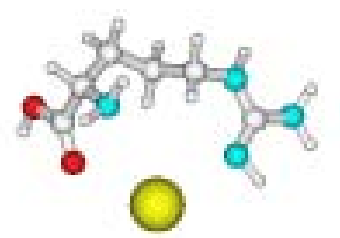

CS_C039

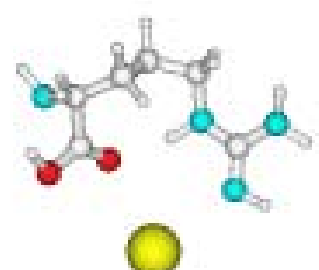

CS_C004

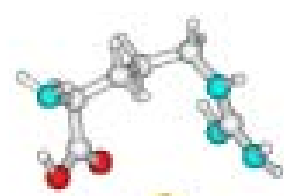

0

$$
\text { CS_C013 }
$$

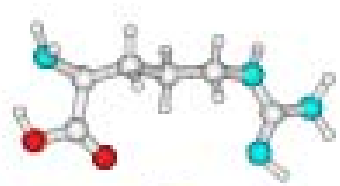

0

CS C025

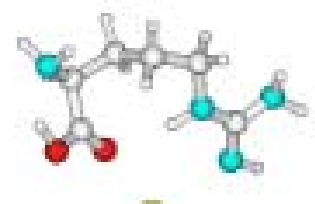

0

CS_C006

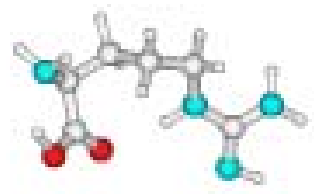

0

CS_C014

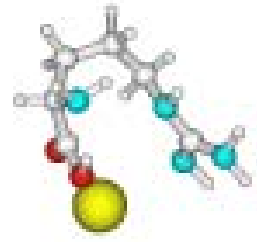

CS_C026 
Scheme 7b. Geometry optimized conformers of $[\mathrm{Arg}+\mathrm{Cs}]^{+}(\mathrm{B} 3 \mathrm{LYP} / 6-31+\mathrm{g}(\mathrm{d}, \mathrm{p}))$.

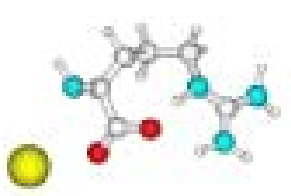

SB_001

0 - क्षे

SB_008

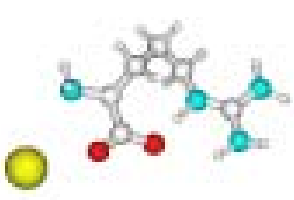

SB_018

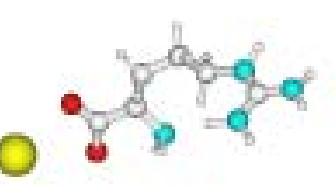

SB_028

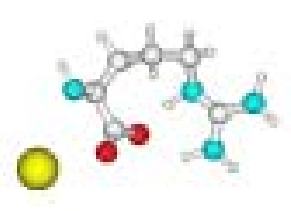

SB_002

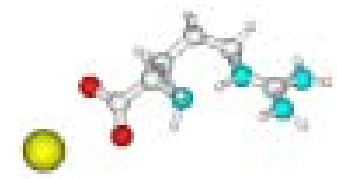

SB_009

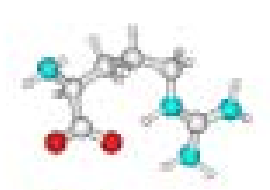

0

SB_019

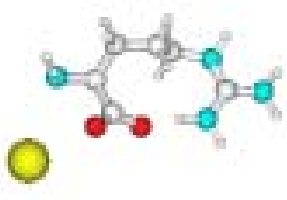

SB_031

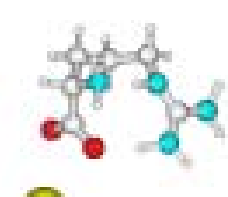

0

SB_003

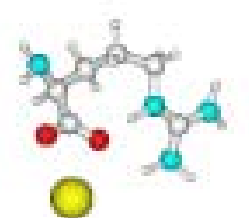

SB_011

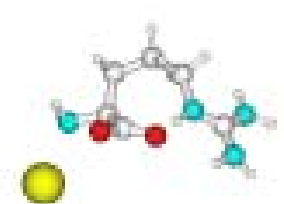

SB_022

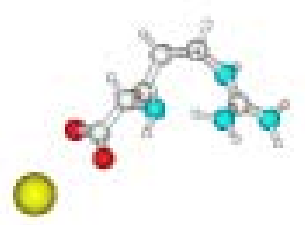

SB_032

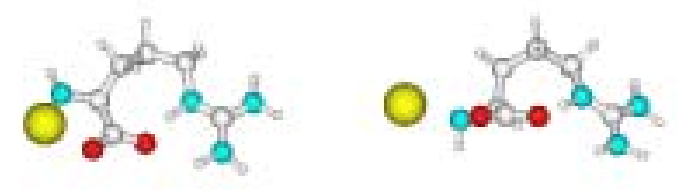

SB_004

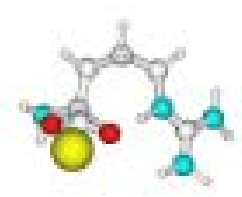

SB_013

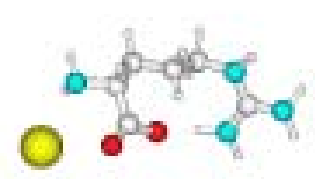

SB_023

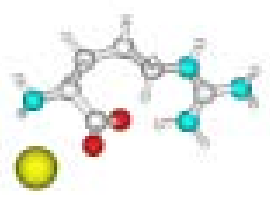

SB_033

$$
\text { SB_014 }
$$

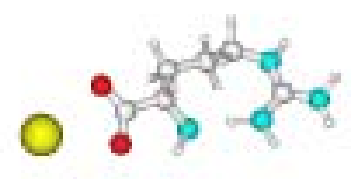

SB_025

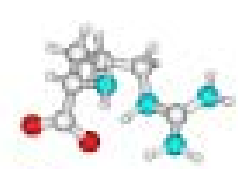

O

SB_006

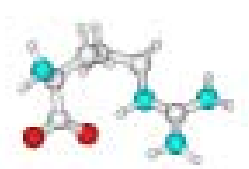

0

SB_015

0 - of

SB_026

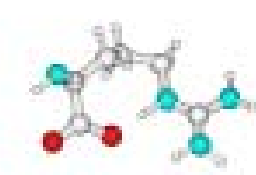

0

SB_016

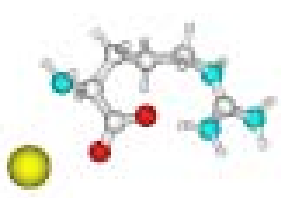

SB_027 
Figure 7. Vibrational spectra for $[\mathrm{Arg}+\mathrm{Cs}]^{+}$conformers from $B 3 \mathrm{LYP} / 6-31+g(d, p)$ calculations. Spectra were constructed using a Gaussian convolution (FWHM=20 $\mathrm{cm}-1$ ) and a scaling factor of 0.98 .
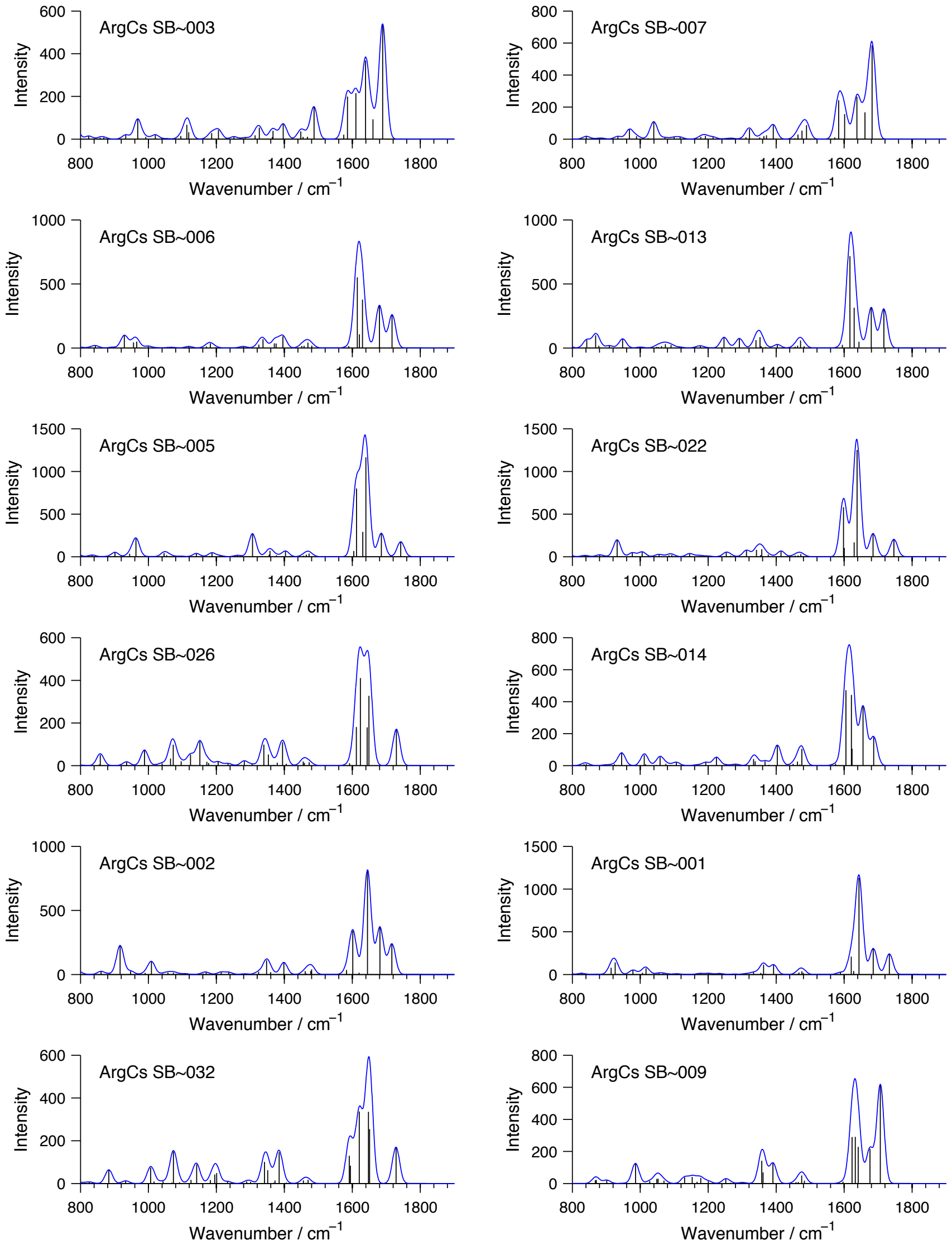
Figure 7 continued.
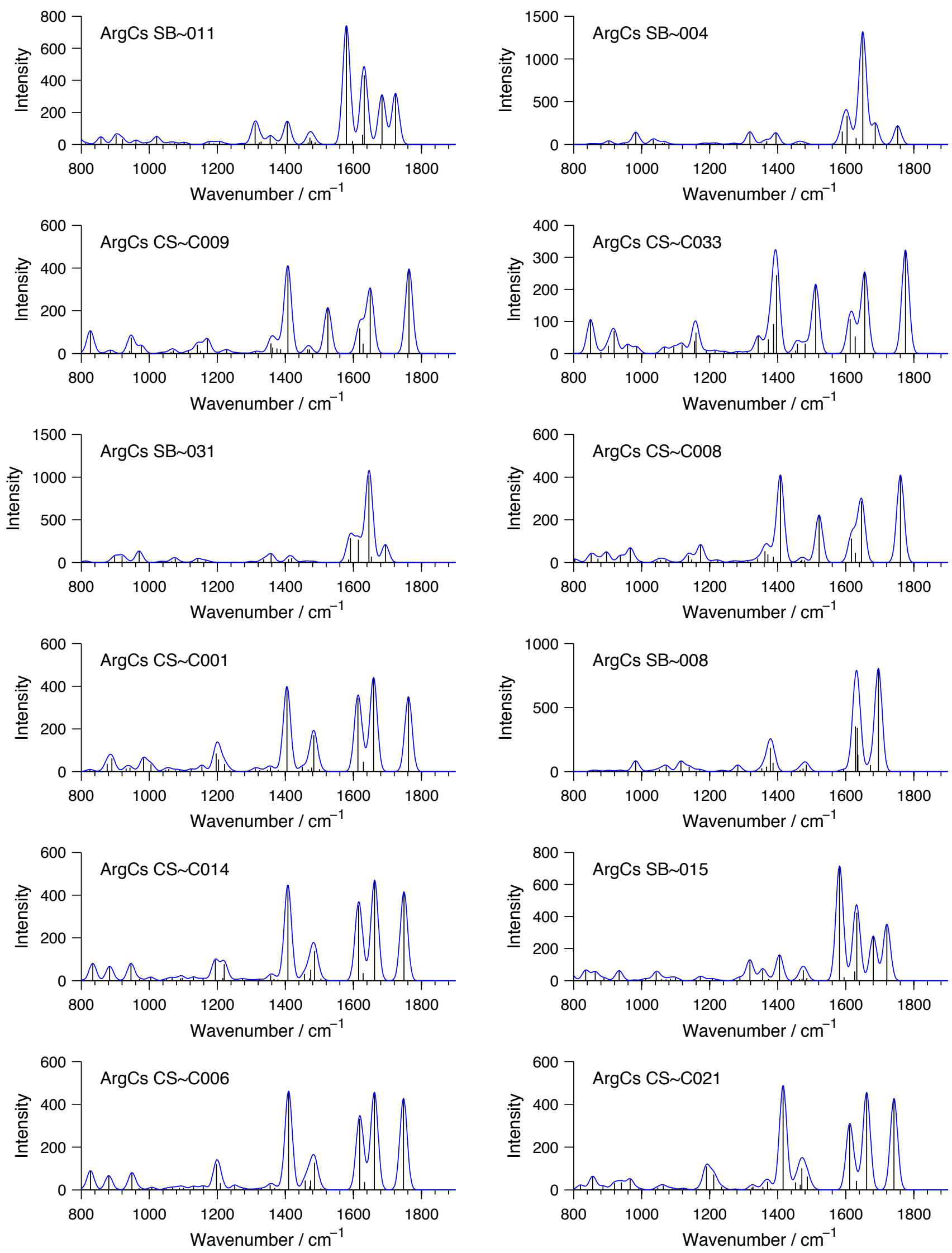
Figure 7 continued.
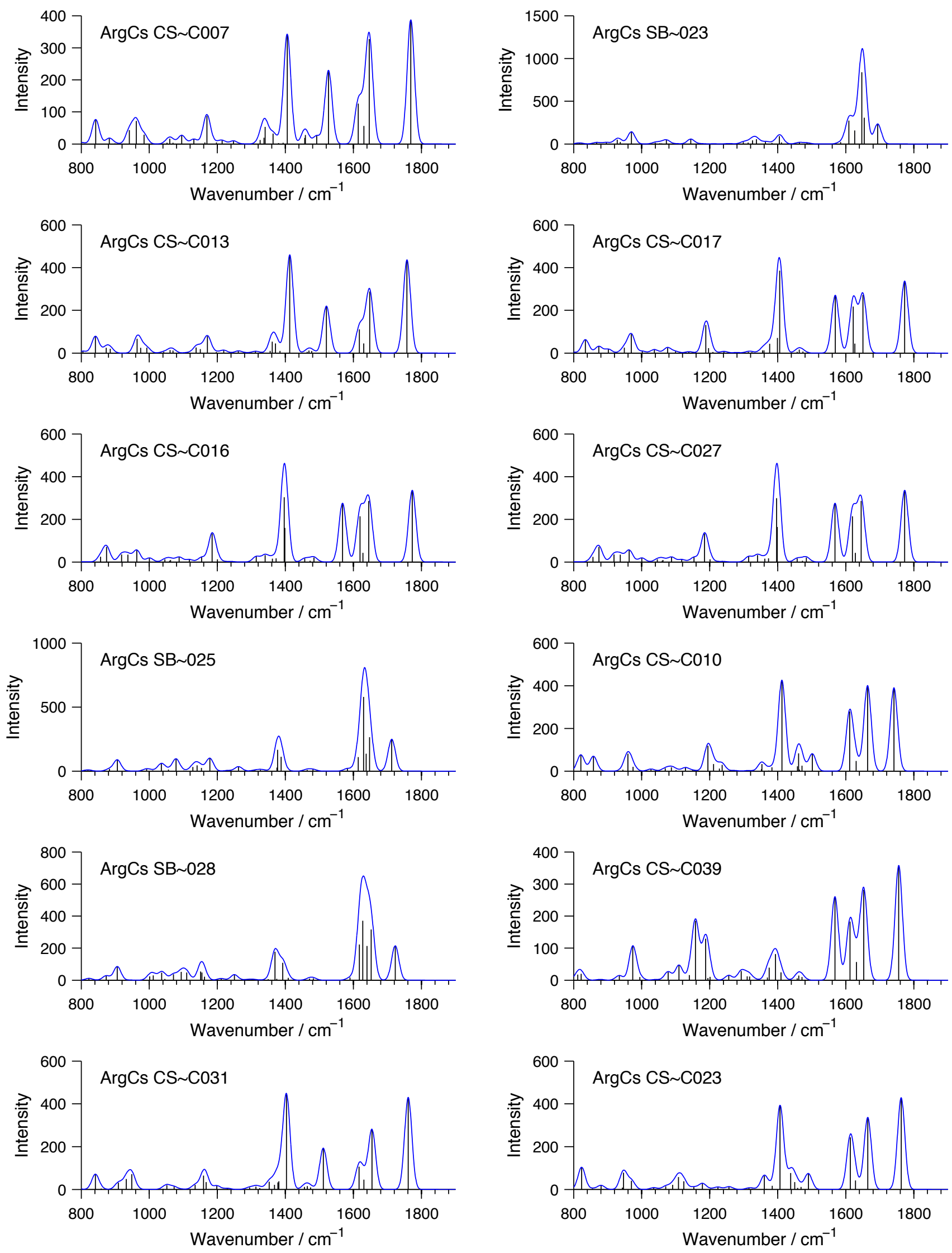
Figure 7 continued.
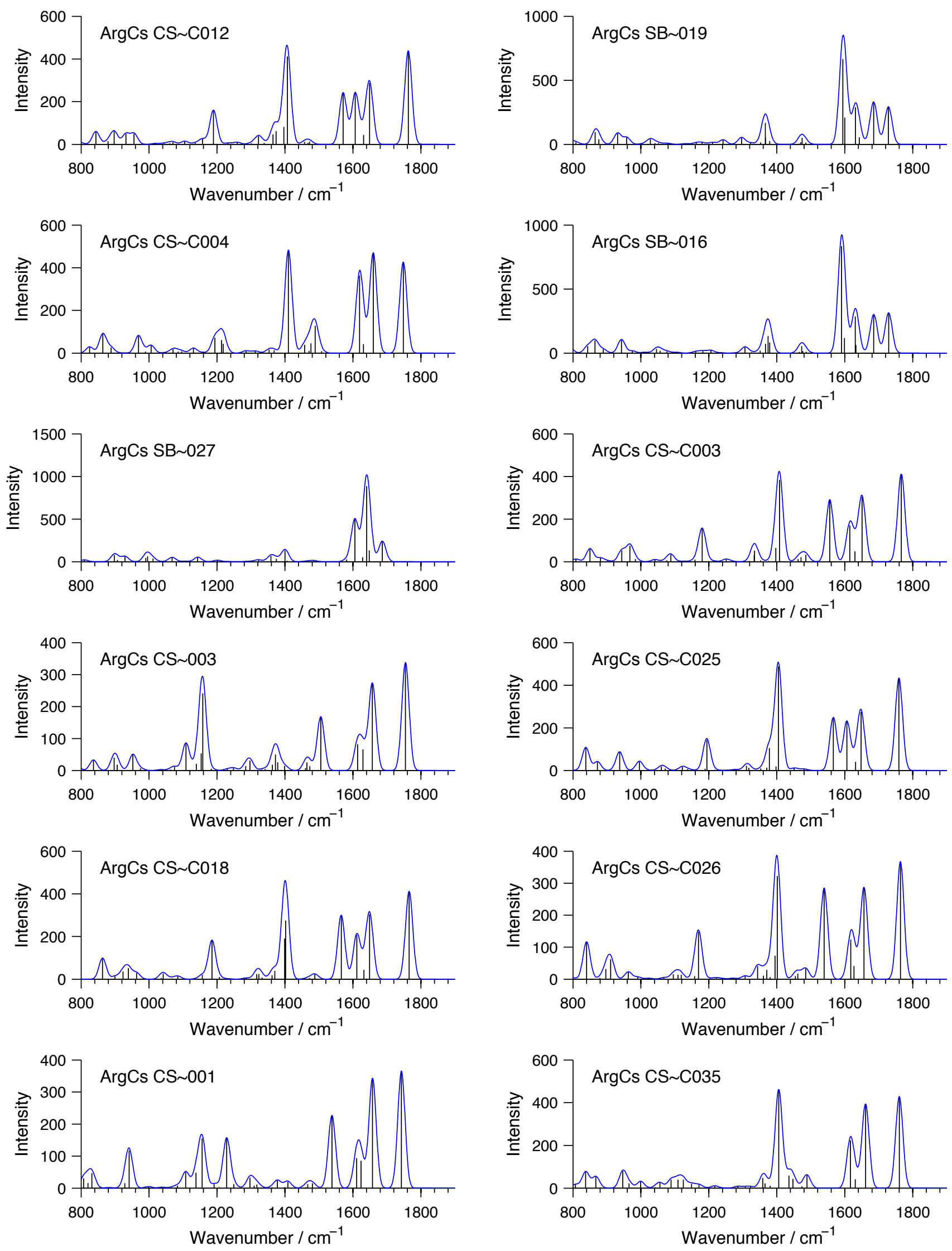
Figure 7 continued.
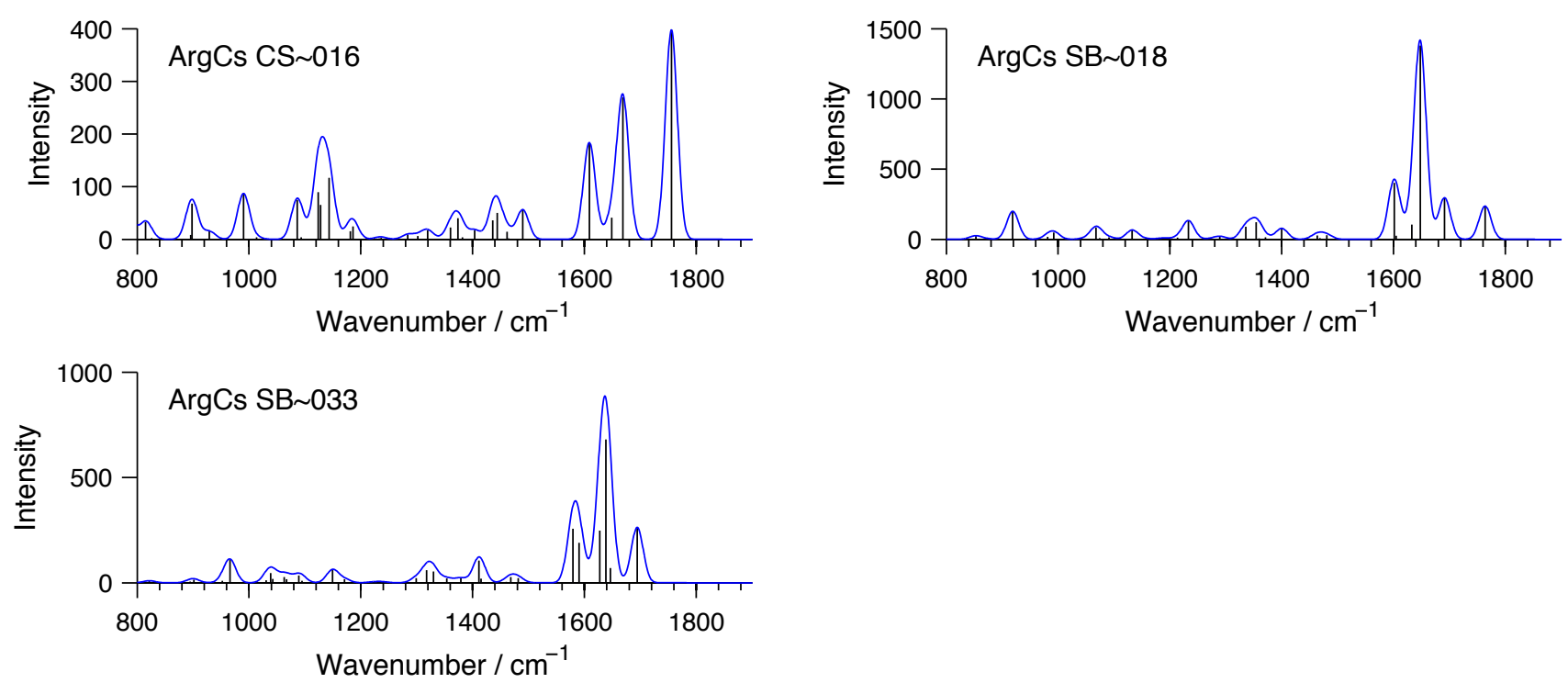
Table 9a. Summary of electronic and thermal energies calculated for B3LYP-optimized conformers of $[\mathrm{Arg}+\mathrm{Ag}]^{+}$

\begin{tabular}{|c|c|c|c|c|c|c|c|c|}
\hline & & & B3LYP & & MP2 & & & \\
\hline ArgAg & Name & Converge & $\begin{array}{c}\Delta E_{\mathrm{o}} / \\
\text { kJ mol-1 }\end{array}$ & $\begin{array}{c}\Delta \mathrm{E}_{\mathrm{o}} / \\
\text { kJ mol-1 }\end{array}$ & $\begin{array}{c}\Delta H(298) / \\
\text { kJ mol-1 }\end{array}$ & $\begin{array}{c}\Delta G(298) / \\
\text { kJ mol-1 }\end{array}$ & $\begin{array}{c}\text { B3LYP E / } \\
h\end{array}$ & $\begin{array}{c}\text { MP2 E / } \\
h\end{array}$ \\
\hline$\overline{C S} \_00 A$ & CS_M & Min & 0.0 & 0.0 & 0.0 & 0.0 & $\begin{array}{l}-753.588135 \\
\end{array}$ & -751.30891 \\
\hline CS_00C & CS_C & Min & 27.0 & 22.0 & 23.3 & 19.5 & -753.576386 & -751.29906 \\
\hline CS_00B & CS_B & Min & 21.3 & 32.8 & 33.1 & 34.1 & -753.579073 & -751.29544 \\
\hline SB_00D & SB_D & Min & 42.8 & 51.7 & 52.3 & 50.4 & -753.570458 & -751.28786 \\
\hline SB_00G & SB_N & Min & 46.2 & 60.8 & 61.7 & 56.7 & -753.569427 & -751.28463 \\
\hline
\end{tabular}

\begin{tabular}{|ll|}
\hline Geometry Optimization & $\mathrm{B} 3 \mathrm{LYP} / 6-31+\mathrm{g}(\mathrm{d}, \mathrm{p})$ \\
Thermal Corrections & $\mathrm{B} 3 \mathrm{LYP} / 6-31+\mathrm{g}(\mathrm{d}, \mathrm{p})$ \\
MP2 Single-point & $\mathrm{MP} 2 / 6-311+\mathrm{g}(2 \mathrm{~d}, 2 \mathrm{p}) / / \mathrm{B} 3 \mathrm{LYP} / 6-31+\mathrm{g}(\mathrm{d}, \mathrm{p})$ \\
\hline
\end{tabular}

Table 9b. Summary of electronic and thermal energies calculated for MPW1PW91optimized conformers of $[\mathrm{Arg}+\mathrm{Ag}]^{+}$

\begin{tabular}{|c|c|c|c|c|c|c|c|c|}
\hline & & & MPW & & MP2 & & & \\
\hline ArgAg & Name & Converge & $\begin{array}{c}\Delta E_{\mathrm{o}} / \\
\text { kJ mol-1 }\end{array}$ & $\begin{array}{c}\Delta E_{\mathrm{o}} / \\
\text { kJ } \mathrm{mol-1}\end{array}$ & $\begin{array}{c}\Delta H(298) / \\
\text { kJ mol-1 }\end{array}$ & $\begin{array}{c}\Delta G(298) / \\
\text { kJ mol-1 }\end{array}$ & $\begin{array}{c}\text { MPW1PW91 } \\
\text { E / h }\end{array}$ & $\begin{array}{c}\text { MP2 E / } \\
h\end{array}$ \\
\hline CS_00A & CS_M & Min & 0.0 & 0.0 & 0.0 & 0.0 & -753.30931 & -751.31000 \\
\hline CS_00C & CS_C & Min & 26.7 & 22.5 & 23.8 & 20.1 & -753.29766 & -751.29995 \\
\hline CS_00B & CS_B & Min & 21.4 & 33.8 & 34.1 & 35.1 & -753.30006 & -751.29603 \\
\hline SB_00D & SB_D & Min & 44.2 & 51.8 & 52.4 & 50.9 & -753.29106 & -751.28884 \\
\hline SB_00I & SB_I & Min & 48.8 & 57.7 & 58.4 & 55.8 & -753.28953 & -751.28683 \\
\hline SB_0GP & SB_G' & Min & 44.1 & 59.3 & 61.0 & 54.9 & -753.29051 & -751.28539 \\
\hline SB_00G & SB_G & Min & 49.5 & 60.2 & 61.0 & 56.1 & -753.28946 & -751.28607 \\
\hline SB_00] & SB_J & Min & 64.4 & 67.0 & 70.1 & 60.4 & -753.28229 & -751.28196 \\
\hline $\mathrm{SB} \_00 \mathrm{H}$ & SB_H & Min & 63.2 & 70.9 & 74.0 & 64.5 & -753.28243 & -751.28021 \\
\hline SB_00F & SB_F & Min & 56.2 & 72.2 & 73.1 & 68.7 & -753.28599 & -751.28057 \\
\hline SB_00K & SB_K & Min & 77.5 & 78.6 & 80.3 & 77.0 & -753.27754 & -751.27781 \\
\hline
\end{tabular}

\begin{tabular}{|ll|}
\hline Geometry Optimization & MPW1PW91/5d 7f \\
Thermal Corrections & MPW1PW91/5d 7f \\
MP2 Single-point & MP2/6-311++g(2d,2p)//MPW1PW91/5d 7f \\
\hline
\end{tabular}


Scheme 8. Geometry optimized conformers of [Arg+Ag] (MPW1PW91/5d 7f).

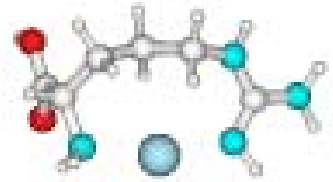

CS_00A

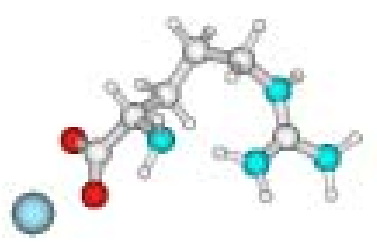

SB_00F

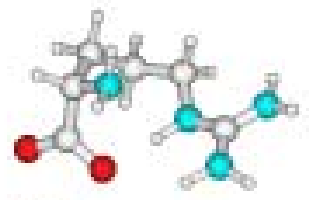

O

SB_00]

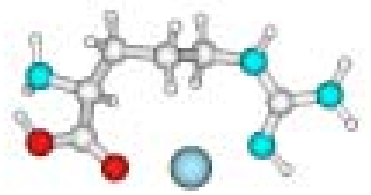

CS_0OB

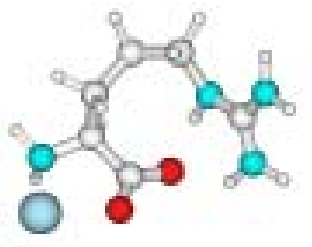

SB_00G

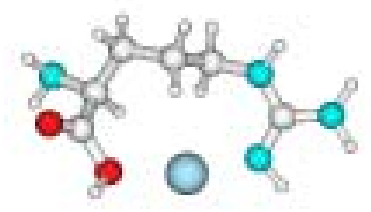

SB_0OK

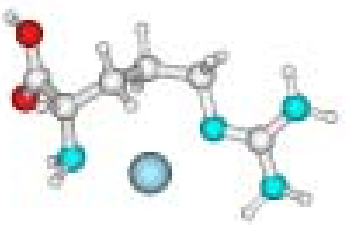

CS_00C

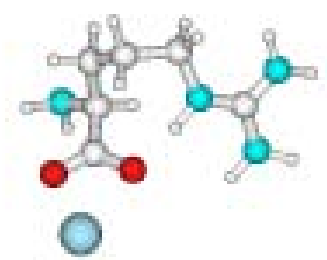

SB_OOH

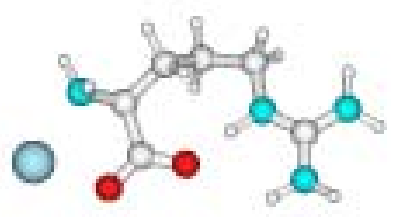

SB_OGP

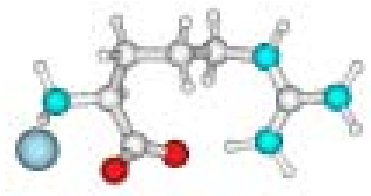

SB_OOI 
Figure 8a. Vibrational spectra for $[A r g+A g]^{+}$conformers from B3LYP/6-31+g(d,p) calculations. Spectra were constructed using a Gaussian convolution (FWHM=20 $\mathrm{cm}-1)$ and a scaling factor of 0.98 .
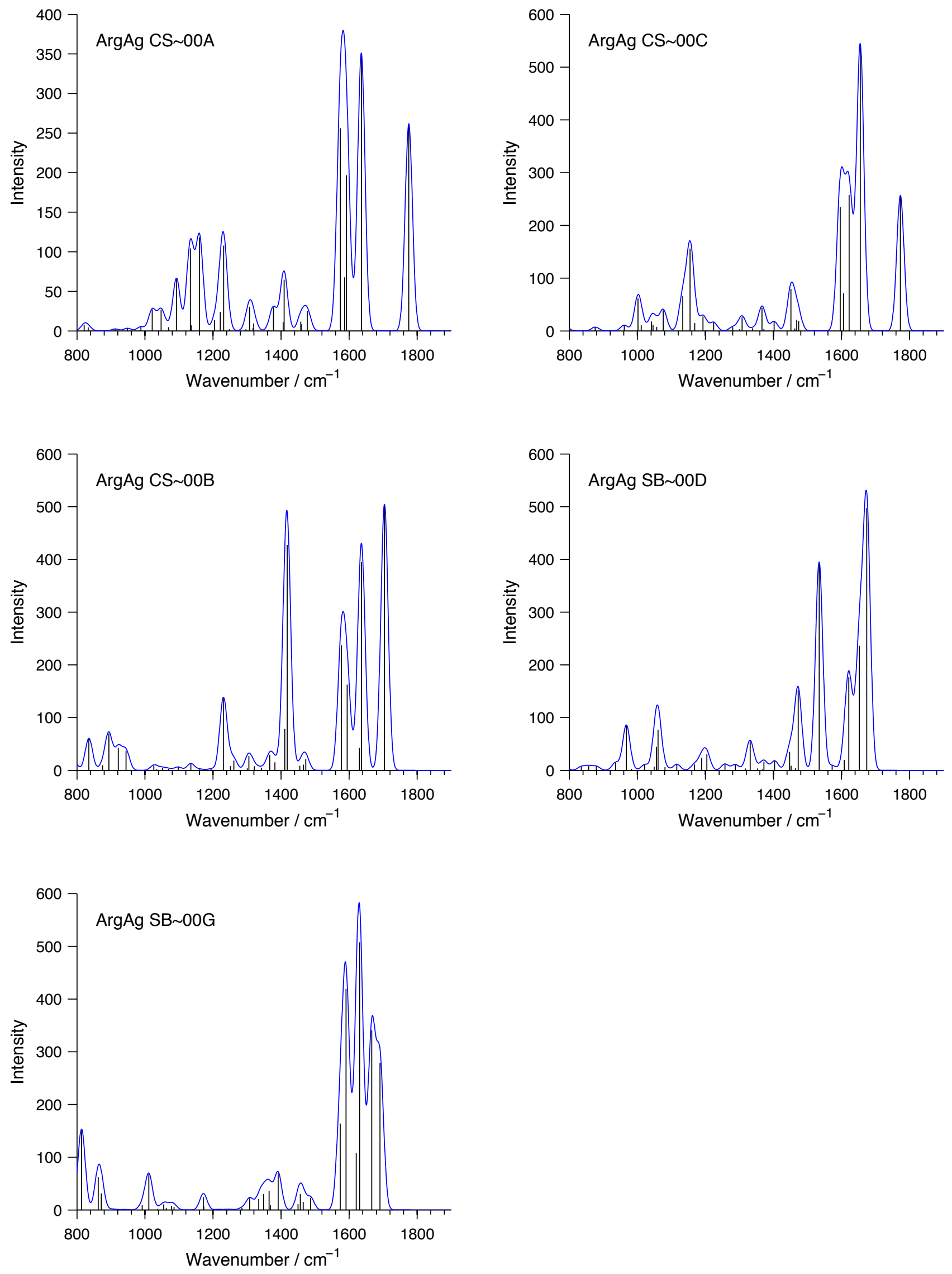
Figure $8 b$. Vibrational spectra for $[\mathrm{Arg}+\mathrm{Ag}]^{+}$conformers from MP21PW91/5d $7 f$ calculations. Spectra were constructed using a Gaussian convolution (FWHM=20 $\mathrm{cm}-1$ ) and a scaling factor of 0.98 .
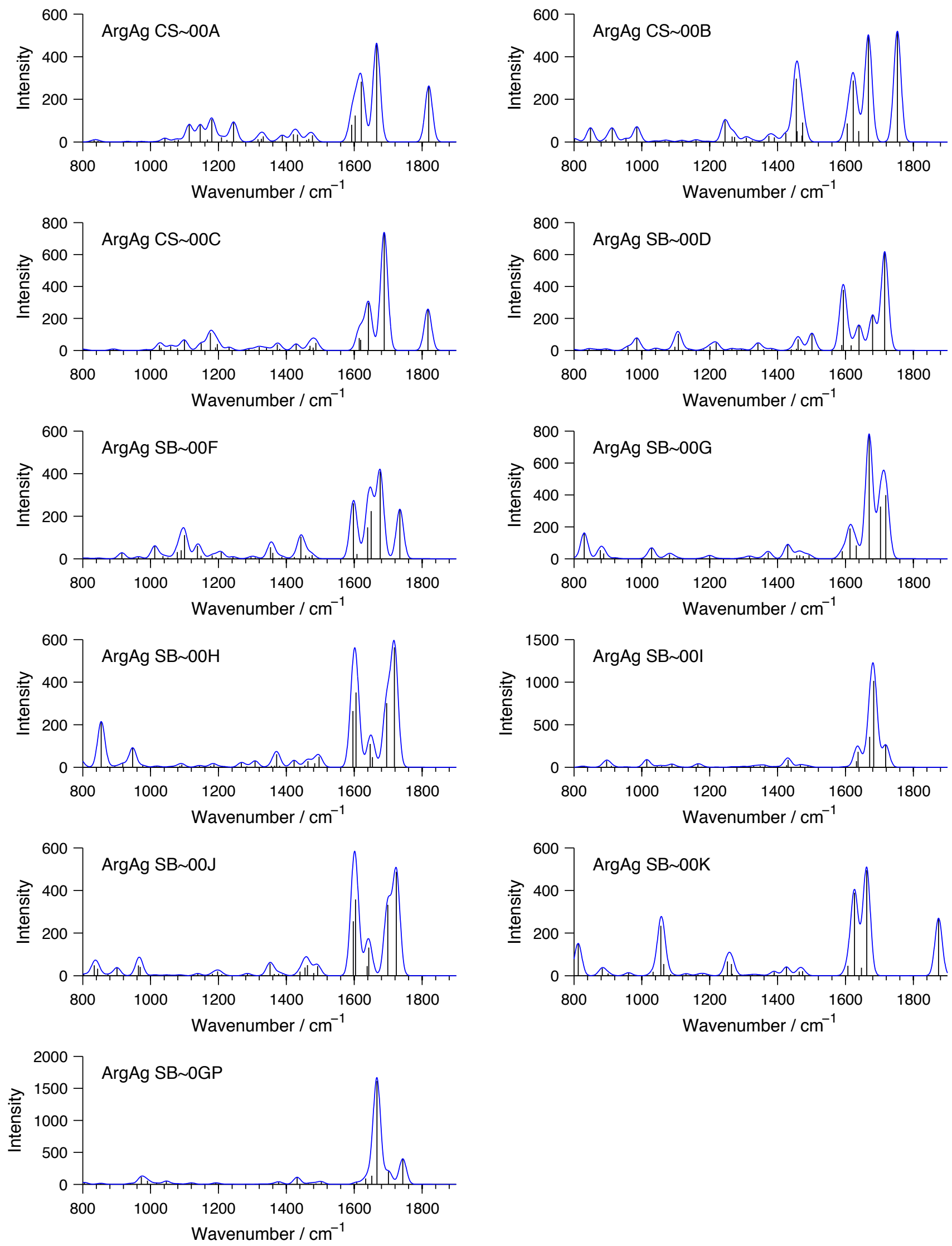


\section{Unabridged References}

(70) Frisch, M. J.; Trucks, G. W.; Schlegel, H. B.; Scuseria, G. E.; Robb, M. A.; Cheeseman, J. R.; J. A. Montgomery, J.; Vreven, T.; Kudin, K. N.; Burant, J. C.; Millam, J. M.; Iyengar, S. S.; Tomasi, J.; Barone, V.; Mennucci, B.; Cossi, M.; Scalmani, G.; Rega, N.; Petersson, G. A.; Nakatsuji, H.; Hada, M.; Ehara, M.; Toyota, K.; Fukuda, R.; Hasegawa, J.; Ishida, M.; Nakajima, T.; Honda, Y.; Kitao, O.; Nakai, H.; Klene, M.; Li, X.; Knox, J. E.; Hratchian, H. P.; Cross, J. B.; Bakken, V.; Adamo, C.; Jaramillo, J.; Gomperts, R.; Stratmann, R. E.; Yazyev, O.; Austin, A. J.; Cammi, R.; Pomelli, C.; Ochterski, J. W.; Ayala, P. Y.; Morokuma, K.; Voth, G. A.; Salvador, P.; Dannenberg, J. J.; Zakrzewski, V. G.; Dapprich, S.; Daniels, A. D.; Strain, M. C.; Farkas, O.; Malick, D. K.; Rabuck, A. D.; Raghavachari, K.; Foresman, J. B.; Ortiz, J. V.; Cui, Q.; Baboul, A. G.; Clifford, S.; Cioslowski, J.; Stefanov, B. B.; Liu, G.; Liashenko, A.; Piskorz, P.; Komaromi, I.; Martin, R. L.; Fox, D. J.; Keith, T.; Al-Laham, M. A.; Peng, C. Y.; Nanayakkara, A.; Challacombe, M.; Gill, P. M. W.; Johnson, B.; Chen, W.; Wong, M. W.; Gonzalez, C.; Pople, J. A. Gaussian 03 Revision D.01, Gaussian Inc. Wallingford, CT, 2004. 